\title{
RNA-seq analysis of Lgr6+ stem cells and identification of an Lgr6 isoform
}

GC van de Glind*1, AN Bastidas Torres*1, WH Zoutman ${ }^{1}$, SAJ van der Zeeuw ${ }^{2}$, SM Kielbasa $^{2}$, FR de Gruijl${ }^{1}$ and CP Tensen ${ }^{\# 1}$

${ }^{1}$ Department of Dermatology, ${ }^{2}$ Department of Medical Statistics and Bioinformatics, Leiden University Medical Center, Leiden, The Netherlands

*Contributed equally to this study

\section{\# Corresponding author}

C.P. Tensen

Leiden University Medical Center

Department of Dermatology

P.O. Box 9600, C3-S

2300 RC Leiden

The Netherlands

Telephone: $(+31)(0) 715266318$

Email: $\underline{\text { C.P.Tensen@lumc.nl }}$

Key words (3-5): Lgr6, stem cells, RNA sequencing, Lgr6 isoform

Word count: 1034 (max 1000, excl. Abstract with 99)

Display items: 1 fig $(\max 2)$

References: 7 ( $\max 9)$

\section{Abstract}

We studied Lgr6 ${ }^{+}$stem cells in experimental UV carcinogenesis in hairless mice. For further characterisation through RNA-seq, these stem cells were isolated by FACS from transgenic hairless mice bearing an EGFP-Ires-CreERT2 reporter cassette inserted into exon 1 of the Lgr6 gene (purity confirmed by human ERT2 expression). Between Lgr6 ${ }^{+}$and Lgr6 ${ }^{-}$basal cells (Tg/wt) 682 RNAs were differentially expressed, indicating stemness and expression of cancer-related pathways in Lgr6' cells. We discovered that suspected 'Lgr6 null' mice (Tg/Tg) expressed RNA of an Lgr6 isoform (delta-Lgr6, lacking 74 N-terminal aa) which could be functional and explain the lack of a phenotype. 
We investigated the fate of a novel class of $\mathrm{Lgr6}^{+}$proliferative stem cells in UV carcinogenesis in hairless mice, a model closely mimicking the development of cutaneous squamous cell carcinoma in man [1]. Using transgenic mice with an EGFP-Ires-CreERT2 reporter cassette inserted into exon 1 of $\operatorname{Lgr} 6$, we found - similar to what was found in haired mice [2] - that Lgr6 ${ }^{+}$stem cells appeared in large clusters in the interfollicular epidermis (IFE) and in very small clusters in the remnants of hair follicles of hairless mice [3]. Contrary to our expectation, the Lgr6 ${ }^{+}$cells dwindled in numbers in the hyperplastic IFE under chronic UV exposure, and did not appear to become tumor-initiating cells (TICs).

Aim: As part of this study we aimed to characterize the $\operatorname{Lgr}^{+}$stem cells further starting with RNA-seq analysis. The two main questions we wanted to address were: a) what is the gene expression profile of $\mathrm{Lgr6}^{+}$stem cells and is there a unique expression of an alternative, more robustly expressed, (surface) marker for the Lgr6 $^{+}$stem cells? And b) what is the status of the Lgr6 gene in mice homozygous for the EGFP-Ires-CreERT2 (Tg/Tg) which are supposedly 'Lgr6 null' mice? Despite this defect these mice appear to be healthy without any apparent phenotype.

Set-up (detailed Material and Methods in Supplement): Epidermal cell suspensions were obtained by trypsin digestion of skin sheets from dorsum, ventrum and flanks. Basal cells were labelled by anti- $\alpha 6$-integrin-PE fluorescent conjugated monoclonal antibodies. We separated (green fluorescent) Lgr6 ${ }^{+}$from Lgr6- basal cells by live-cell FACS (Figure S1). For robust sequencing, extracted RNA of 2 mice of similar age (male and female, both either $\mathrm{Tg} / \mathrm{wt}$ or $\mathrm{Tg} / \mathrm{Tg}$ ) was pooled. For RNA-seq, pooled RNA samples (450 $\mathrm{ng}$ ) were processed by an Illumina HiSeq-2000 (Illumina Inc., San Diego, CA, USA).

Results \& Discussion: RNA-seq showed 682 genes to be differentially expressed (291 up, 391 down) with adjusted p-values (FDR) < 0.05 comparing Lgr6 $^{+}$with Lgr6 $^{-}$basal (Tg/wt) cells (Table S1 sorted according to FDR). Figure 1a depicts a heat map of the top most 
differentially expressed genes (35 up and 35 down). Successful purification of Lgr6 ${ }^{+}$basal cells could be confirmed by exclusive expression of ERT2 (Figure S2), an engineered fragment of human ESR1 coding for the tamoxifen binding domain. Also, the Lgr6 expression was much higher in the sorted Lgr6 $^{+}$cells, but Lgr6 ${ }^{-}$cells were not completely devoid of $\operatorname{Lgr6}$ transcripts (Figure S3; verified by qPCR). This could be due to stochastic hypermethylation of the promoter of the reporter cassette [3] which could silence EGFP expression and thus cause some leakage of expression of the accompanying wt $\operatorname{Lgr6}$ allele into the sorted $\operatorname{Lgr6}^{-}(\mathrm{Tg} / \mathrm{wt})$ cells. This would evidently affect the contrast in expression between the Lgr6 ${ }^{+}$and Lgr6 sorted fractions.

As anticipated, the $\operatorname{Lgr}^{+}$samples showed increased expression of several stem cell-related genes (e.g. Kitl, Gas family members, Ctgf) (see Figure 1a), and decreased expression of bulge stem cell markers (e.g. Tcf4, Fzd3, Runxl, Sox9, Tnc). DAVID and KEGG pathway mapping supported stemness of Lgr6 ${ }^{+}$cells by increased expression of genes involved in pluripotency and decreased expression of genes controlling metabolic pathways (Supplementary Table S2). Also, highly significant increased expression of pathways related to cancer, particularly basal cell carcinoma, including the hedgehog pathway, was observed (Supplementary Table S2). In accordance with Lgr6 enhancement of Wnt signaling, several of these 'Wnt genes' (e.g. Wnt7b, Lgr6, Znrf3, Axin2; the latter verified by qPCR) showed increased expression in Lgr6 $^{+}$cells (see Figure 1c). Of Rspo1-4 as ligands of Lgr6, only Rspo3 was found to be expressed in $\operatorname{Lgr6}^{+}$and $\operatorname{Lgr6}^{-}$cells.

Strikingly, many of the top 35 RNAs with lower expression in the Lgr6 ${ }^{+}$vs Lgr6 ${ }^{-}$samples were non-coding (15/35). The most strongly differentially expressed protein-coding genes were Sostdc1 and Fst (Figure 1a, arrow heads; down in Lgr6 $^{+}$); products of which inhibit signaling of BMPs and TGF- $\beta$ family members, respectively. Among the top of higher expressed protein-coding genes in Lgr6 ${ }^{+}$cells (e.g. Igfbp3, Adam8, Nat8l, Skint7, Crispld2, 
Adamts14, Ngfr, Slc2a12) some may code for robust alternative surface markers of Lgr6 $^{+}$ stem cells. From scrutiny of genecard.org and proteinatlas.org, Ngfr (low affinity nerve growth factor receptor) and Slc2a12 (glucose transporter 12), for example, would appear to be promising candidates: they are membrane-bound and show the anticipated partial to sparse staining of epidermal basal cells (in human skin with antibodies CAB000143 and 001995 for Ngfr, HPA031593 for Slc2a12). Further research is evidently called for on isolating from human skin cells bearing alternative markers and expressing LGR6.

After initial presentation of our work [4], Füllgrabe et al [2] published results on RNA-seq of Lgr6 $^{+}$versus Lgr6 ${ }^{-}$cells from hair follicles and IFE of haired mice. Surprisingly, they found no distinct expression signature of Lgr6+ cells, and only differential expression in hair follicles, not in IFE (particularly not for Lgr6). Our samples contained mainly Lgr6 $^{+}$cells from IFE and did show extensive differential expression. This may simply be attributed to the fact that our samples contained 450 fold more RNA and our read counts were at least 100x higher allowing more reliable quantification of low abundant transcripts and more rigorous statistical testing.

Contrary to expectation [5], RNA-seq results from homozygous knock-in mice ( $\mathrm{Tg} / \mathrm{Tg}$ ) showed transcripts of Lgr6. These skipped exon 1 (with the knock-in cassette), started in close proximity to exon 2 (see Figure S4) and ran on through exon 18 (confirmed by qPCR, supplementary Table S3 for primers). This coded for an N-terminal truncated protein (deltaLgr6) lacking 74 amino acids compared to full length Lgr6. A perfectly matching tryptic peptide (minus the N-terminal methionine) was found in dataset PRDB000032 (proteomicsDB) [6], indicating that the human delta-LGR6 homologue is expressed (see Figure S6) with post-translational removal of the N-terminal methionine. Based on sequence homology with LGR4, critical R-spondin binding residues in the ectodomain of LGR6 (aa 121-263) [7] are completely preserved in delta-LGR6/Lgr6. Thus, delta-Lgr6 could be fully 
functional and explain the lack of a phenotype in homozygous knock-in mice ( $\mathrm{Tg} / \mathrm{Tg})$, suspected 'Lgr6 null' mice. Biological implications of truncated delta-LGR6 need to be explored.

We conclude that the RNA-seq data provided extensive leads on further characterization of Lgr6+ stem cells in the epidermis and on possible switching to expression of a delta-Lgr6 homologue.

\section{Acknowledgement}

This research was supported by a grant of the Dutch Cancer Society (UL2010-4812). We

thank prof. Hans Clevers and his team for starting us up with the transgenic mice and Heggert

Rebel for assistance in performing the experiments. Paul Hensbergen suggested and supported searching the proteomics DB database. Yavuz Ariyurek carried out the RNA-seq.

\section{Accession Numbers}

RNA-seq data available at http://www.ebi.ac.uk/ena/data/view/PRJEB15002

\section{References:}

1. Chitsazzadeh V, Coarfa C, Drummond JA, Nguyen T, Joseph A, Chilukuri S, Charpiot E, Adelmann CH, Ching G, Nguyen TN, Nicholas C, Thomas VD, Migden M, MacFarlane D, Thompson E, Shen J, Takata Y, McNiece K, Polansky MA, Abbas HA, Rajapakshe K, Gower A, Spira A, Covington KR, Xiao W, Gunaratne P, Pickering C, Frederick M, Myers JN, Shen L, Yao $\mathrm{H}$, Su X, Rapini RP, Wheeler DA, Hawk ET, Flores ER, Tsai KY. Cross-species identification of genomic drivers of squamous cell carcinoma development across preneoplastic intermediates. Nature communications. 2016;7:12601

2. Füllgrabe A, Joost $S$, Are A, Jacob T, Sivan U, Haegebarth A, Linnarsson S, Simons BD, Clevers $H$, Toftgård R, Kasper M. Dynamics of Lgr6(+) Progenitor Cells in the Hair Follicle, Sebaceous Gland, and Interfollicular Epidermis. Stem cell reports. 2015;5:843-855.

3. van de Glind GC, Rebel HG, Out-Luiting JJ, Zoutman W, Tensen CP, de Gruijl FR. Lgr6+ stem cells and their progeny in mouse epidermis under regimens of exogenous skin carcinogenesis, and their absence in ensuing skin tumors. Oncotarget 2016;7:86740-86754.

4. Bastidas AN, van de Glind G, Ramos da Cunha Lima I, Rebel H, de Gruijl F, Tensen K. The transcriptome of Lgr6+ epidermal stem cells in mouse skin. J Invest Dermatol. 2015;135:S68. 
5. Snippert HJ, Haegebarth $A$, Kasper $M$, Jaks V, van Es JH, Barker $N$, van de Wetering $M$, van den Born $M$, Begthel $H$, Vries RG, Stange DE, Toftgård R, Clevers H. Lgr6 marks stem cells in the hair follicle that generate all cell lineages of the skin. Science. 2010;327:1385-1389.

6. Munoz J, Low TY, Kok YJ, Chin A, Frese CK, Ding V, Choo A, Heck AJ. The quantitative proteomes of human-induced pluripotent stem cells and embryonic stem cells. Mol Syst Biol. 2011;7:550.

7. Xu JG, Huang C, Yang Z, Jin M, Fu P, Zhang N, Luo J, Li D, Liu M, Zhou Y, Zhu Y. Crystal structure of LGR4-Rspo1 complex: insights into the divergent mechanisms of ligand recognition by leucine-rich repeat G-protein-coupled receptors (LGRs). J Biol Chem. 2015;290:2455-2465.

a

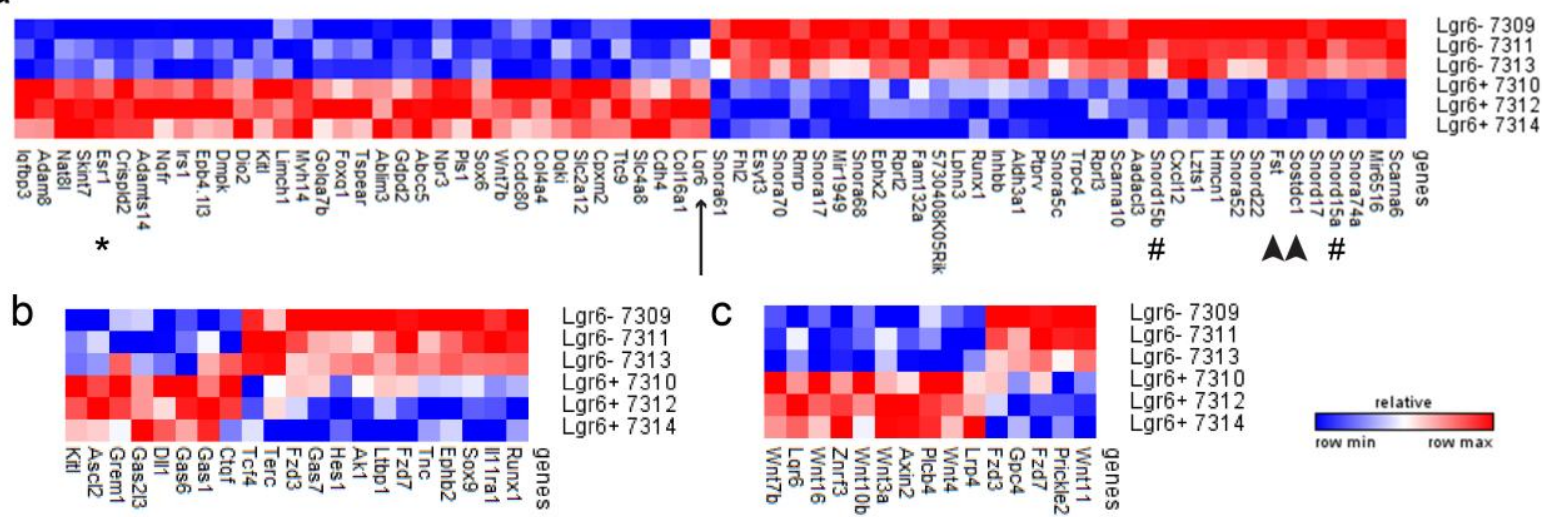

Figure 1. Heatmaps showing differentially expressed genes between Lgr6+ and Lgr6cells from Tg/wt mice. (a) top 35 up- and downregulated genes (ranked on FDR) ${ }^{*}=$ Esr1, arrow $=$ Lgr6, arrow heads $=$ Fst and Sostdc1, $\#=$ Snord15a and $b$ non-coding in protein-coding Rps3 gene, see Fig. S5). (b) shows stem cell-related genes and (c) genes from the Wnt pathway. 


\section{SUPPLEMENTARY INFORMATION part 1 (M\&M and Figures)}

RNA-seq analysis identifies an Lgr6 isoform and gene expression pattern characterizing adult skin stem cells

GC van de Glind*1, AN Bastidas Torres*1, WH Zoutman ${ }^{1}$, SAJ van der Zeeuw ${ }^{2}$, SM Kielbasa $^{2}$, FR de Gruijl $1^{1}$ and CP Tensen ${ }^{\# 1}$

${ }^{1}$ Department of Dermatology, Leiden University Medical Center, Leiden, The Netherlands

${ }^{2}$ Department of Medical Statistics and Bioinformatics, Leiden University Medical Center, Leiden, The Netherlands

*Contributed equally to this study

\section{MATERIALS AND METHODS}

Mice

Lgr6-EGFP-Ires-CreERT2/R26R-LacZ transgenic mice (kindly provided by Prof. Hans Clevers) were backcrossed into a hairless background using Crl:SKH1-HR hairless mice (Charles River, Sulzfeld, Germany). Both homozygous and heterozygous transgenic "Lgr6 mice" were obtained, not showing any phenotypic alterations despite Lgr6 gene disruption. Male and female mice were used in the experiments (see for details supplementary Table S4). They were kept individually in Macrolon type 1 cages at $25 \pm 2{ }^{\circ} \mathrm{C}$ with a humidity of $50 \%$ in a 12 hours light-12 hours dark cycle during experiments. Standard chow and tap water were available ad libitum. As legally required, all mouse experiments were performed with the approval of the Leiden University Medical Centers' ethics committee (approval number DEC 10229) and executed according to EU regulations on animal experiments (Directive 2010/63/EU).

\section{Cell suspension preparation, antibody labelling and FACS sorting}

Mice were sacrificed by $\mathrm{CO} 2$ asphyxiation, and back and belly skin was removed. The skin sample was incubated epidermal side up in $25 \mathrm{ml}$ PBS containing trypsin $(0.25 \%$; T4799, Sigma-Aldrich, St. Louis, MO, USA) + D-glucose (0.1\%; G5400, Sigma-Aldrich) over night at $4^{\circ} \mathrm{C}$ and at $37^{\circ} \mathrm{C}$ for $15 \mathrm{~min}$. The epidermis was scraped off of the dermis and minced. The epidermis was digested using $8 \mathrm{ml}$ PBS containing trypsin $(0.25 \%)+\mathrm{D}$-glucose $(0.1 \%)$ and repeated pipetting. $16 \mathrm{ml}$ PBS with 5\% BSA (A9647, Sigma-Aldrich), DNAse I (200 Kunitz units/ml; DN25, Sigma-Aldrich) and $\mathrm{MgCl} 2.6 \mathrm{H} 2 \mathrm{O}$ (5mM; M2393, Sigma-Aldrich) was added and the suspension was put through a $70 \mu \mathrm{m}$ cell strainer. The strained cell suspension was incubated for 30 minutes at RT. Cells were spinned down and resuspended in $300 \mu \mathrm{l}$ of supernatant. $8 \mathrm{ml}$ PBS with BSA (1\%), DNAse I (50 Kunitz units/ml) and $\mathrm{MgCl} 2.6 \mathrm{H} 2 \mathrm{O}$ $(5 \mathrm{mM})$ was added, spinned down and again resuspended in $300 \mu \mathrm{l}$ of the supernatant. The volume was brought to $2 \mathrm{ml}$ with PBS containing BSA (0.2\%), DNAse I (50 Kunitz units/ml) and $\mathrm{MgCl} 2.6 \mathrm{H} 2 \mathrm{O}(5 \mathrm{mM})$. Back and belly cell suspensions of the same animal were combined and cell viability and cell numbers were determined.

With this method we mainly isolated Lgr6+ cells from interfollicular epidermis where they were abundantly present in large EGFP-fluorescent clusters 10, whereas only very few EGFP+ cells were visible in the hair follicle remnants. For FACS analysis, cell suspensions were stained with anti- $\alpha 6$-integrin-PE conjugated monoclonal antibodies (CD49f clone GoH3, 
BD Bioscience, San Jose, CA, USA) for 1 hour at $4^{\circ} \mathrm{C}$. Cells were sorted using a FACSAria III cell sorter (BD Bioscience) on the basis of single cell detection (pulse width), viability/integrity (scatter profile) and both EGFP and PE fluorescence. Sorting of $\mathrm{PE}+$ /EGFP+ and PE+/ EGFP- cells (Lgr6+ and Lgr6- basal cells, respectively) was accomplished through very strict gating settings as shown in Supplementary Figure S1. Sorted cells were collected in sheath fluid on ice. FlowJo software version 7.2.5 (FlowJo, Ashland, OR, USA) was used to obtain FACS plots of raw data. RNA extraction was performed immediately after each sorting procedure.

\section{RNA isolation}

Total RNA from sorted cells was extracted by using RNeasy Mini kit (74106, Qiagen, Hilden, Germany), which included on-column DNase digestion. All samples were eluted in $35 \mu \mathrm{l}$ of RNAse-free water. RNA concentration and purity were determined using a Nanodrop 1000 spectrophotometer (Thermo Fisher Scientific, Wilmington, DE, USA). In addition, integrity of RNA molecules was evaluated by using Agilent RNA 6000 Pico Kit on a 2100 Bioanalyzer (Agilent Technologies, Santa Clara, CA, USA). RNA samples with RIN scores above 8 were used for subsequent RNA-seq sample preparations. RNA isolated from female and male cell suspensions of matched ages $(4,12,23$ weeks) were pooled pairwise; no evident differences in expression profiles.

\section{RNA precipitation, library preparation and RNA deep sequencing}

For RNA-seq, eight total RNA samples (450 ng) were processed. These samples were composed of 2 individual mice (a male and a female) bearing the same transgene zygosity. Combined RNA samples were concentrated by ethanol-mediated precipitation and resuspended in $10 \mu \mathrm{l}$ RNAse-free water for library preparation. Eight individual barcoded libraries were made, normalized and pooled to be sequenced in the same run. Library preparation (rRNA depletion by Ribo-zero magnetic kit, Illumina) and paired-end sequencing of 2 times $100 \mathrm{bp}$ were performed by the Leiden Genome Technology Center (LGTC). Sequencing was performed on a Illumina HiSeq-2000 (Illumina Inc., San Diego, CA, USA) following the instructions of the manufacturer.

\section{Data processing}

RNA-seq data was processed using bioinformatics pipelines of the Sequence Analysis Support Core (SASC) of our institute, LUMC, according following method.

Sequencing adapters in the FastQ files were detected using FastQC version 0.10.1 and removed using the cutadapt tool (version 1.4.2). Base quality trimming was performed using the sickle tool (version 1.200). RNA paired-end reads where aligned to Mus musculus (murine) reference genome version mm10. (GSNAP version 2014-05-15; novel splicing flag set to true). The resulting alignment file was compressed, indexed, and name-sorted using samtools tool (version 0.1.19-44428cd). The count table was generated using htseq-count (HTSeq suite version 0.6.1p1). The UCSC genePredToGtf generated RefSeq annotation (raw database dump of the refGene table) was used as a GTF reference.

With this method, we were able to identify differentially expressed coding and (long) non coding transcripts.

\section{Detection of differential mRNA and IncRNA expression}

Differential gene expression analysis was performed using edgeR 12 and R/Bioconductor (https://www.R-project.org/, http://www.bioconductor.org). The TMM method 13 was used to normalize gene counts. We selected statistically significantly differentially expressed genes 
based on false discovery rates (FDR) obtained with the Benjamini-Hochberg procedure (correcting for multiple testing).

\section{Visualization}

Sequencing results were visualized using the Integrative Genomics Viewer (IGV) 14. Heatmaps depicting $\log 2$ transformed relative differentially expressed genes were created using Gene-E (Broad institute,

www.broadinstitute.org/cancer/software/GENE-E/ accessed September 9 2016)

\section{Functional gene clustering}

Biochemical pathways were analyzed using the web-based annotation tool DAVID v 6.8 (Database for Annotation, Visualization and Integrated Discovery; 15) to cluster statistically significantly differentially regulated genes by their common functionality. Upregulated and downregulated genes were subjected to DAVID analysis as two separate entities.

\section{Reverse transcription and qPCR}

Total RNA was isolated from frozen skin using the RNeasy mini kit (74106, Qiagen, Hilden, Germany) including on-column DNase treatment. cDNA was synthesized from $1 \mu \mathrm{g}$ total RNA using the iScript ${ }^{\mathrm{TM}}$ cDNA Synthesis Kit (170-8891, Biorad, Veenendaal, The Netherlands) according to manufacturer's instructions. qPCR for the different exons of Lgr6 was performed using the SYBR Green Supermix (Bio-Rad) on the CFX $384^{\mathrm{TM}}$ real-time PCR detection system (Biorad). Cycle parameters were as follows: Hot start for 3 minutes at $95^{\circ} \mathrm{C}$; denaturation for 15 seconds at $95^{\circ} \mathrm{C}$, annealing and extension for 30 seconds at $60^{\circ} \mathrm{C}$ for 40 cycles. Specificity of the PCR products was confirmed by melting curve analysis. Data was normalized against reference genes Cyc1 and Ddx52 (see supplementary Table S3 for primers used) using the $\Delta \Delta \mathrm{Cq}$ method (Livak and Schmittgen, 2001) and is presented as relative mRNA expression (see supplementary figure $\mathrm{S} 4$ ). 
a

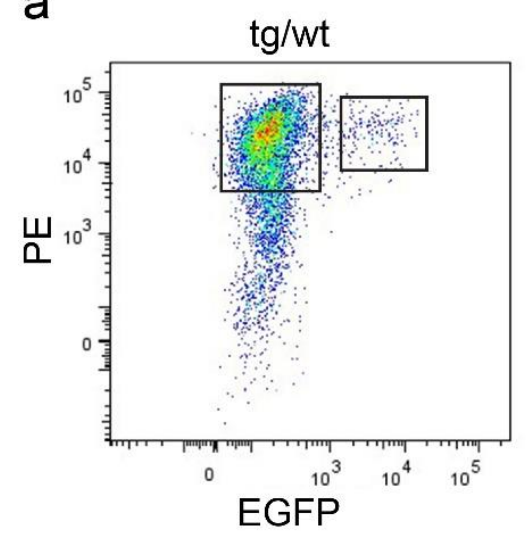

b

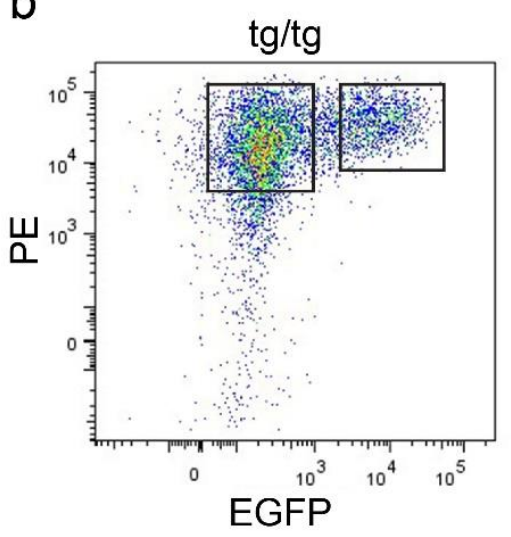

C

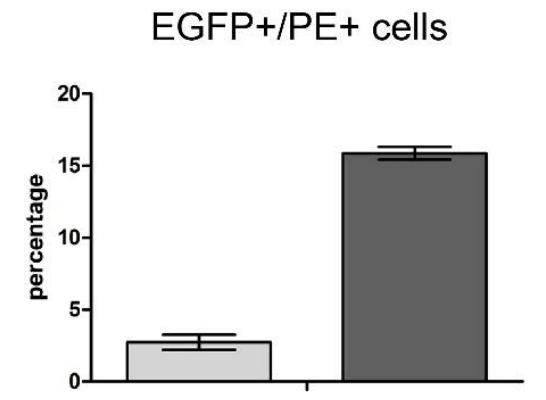

$\mathrm{tg} / \mathrm{wt} \quad \mathrm{tg} / \mathrm{tg}$

Figure S1 FACS sorting of Lgr6+ cells from murine skin The FACS plots show three main distinguishable areas: apparent $\mathrm{Lgr6}^{+}$epidermal stem cells $\left(\mathrm{PE}^{+} / \mathrm{EGFP}^{+}\right)$, cells that are negative for both markers $\left(\mathrm{PE}^{-} / \mathrm{EGFP}^{-}\right)$and basal layer epidermal cells $\left(\mathrm{PE}^{+} / \mathrm{EGFP}^{-}\right)$; the latter group constituting the largest fraction of cells in suspension. a) FACS sorting of a heterozygous Lgr6 $^{+}$knock-in mouse and b) a homozygous Lgr6 $^{+}$knock-in mouse c) $\mathrm{EGFP}^{+} / \mathrm{PE}^{+}$cells constitute on average $2.5 \%$ and $15 \%$ of all cells in suspension in heterozygous and homozygous knock-in animals, respectively. Error Bars: Standard Error, $\mathrm{Tg} / \mathrm{wt}(\mathrm{n}=11), \mathrm{Tg} / \mathrm{Tg}(\mathrm{n}=4)$. 
a

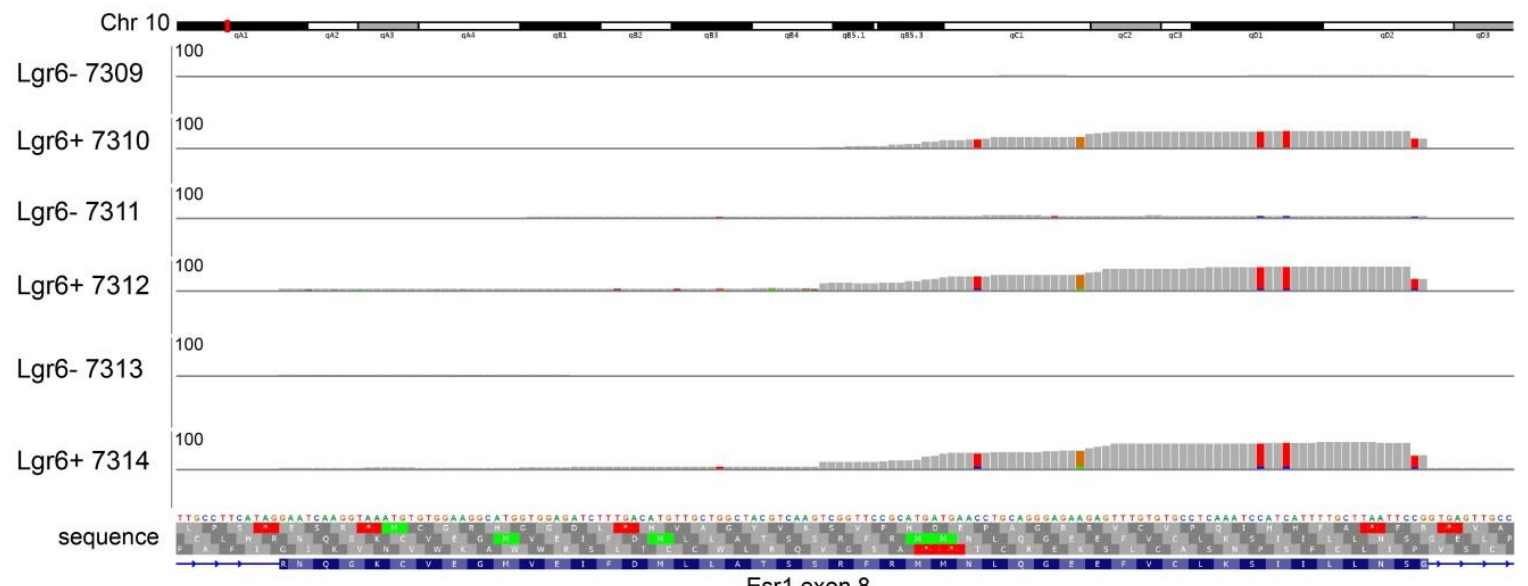

b

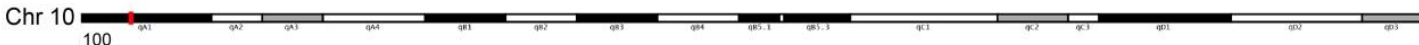

Lgr6- 7309

Lgr6+ 7310

100

Lgr6- 7311

Lgr6+ 7312

100

Lgr6- 7313

100

Lgr6+ 7314

sequence

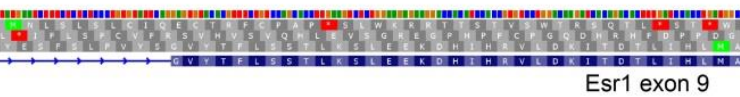

Figure S2: RNA-seq reads mapped to Exon 8 (chr10: 4969159-4969292; a) and 9 (chr10:4997830-4998013; b) of the Esr1 gene for all heterozygous transgenic samples. Screenshot from IG viewer of the base counts for both exons are shown (y-axis = 100), for Lgr6+ stem cells (even numbered samples) and for epidermal basal cells (Lgr6-, odd numbered samples). Coloured bars in the cumulative counts mark bases deviant from the reference mouse sequence but in accordance with the human sequence. Lgr6+ stem cells clearly express human ESR1 (present as ERT2 in the EGFP-Ires-CreERT2 cassette) while Lgr6- cells show very low counts mainly from (endogenous) Esr1. 
a

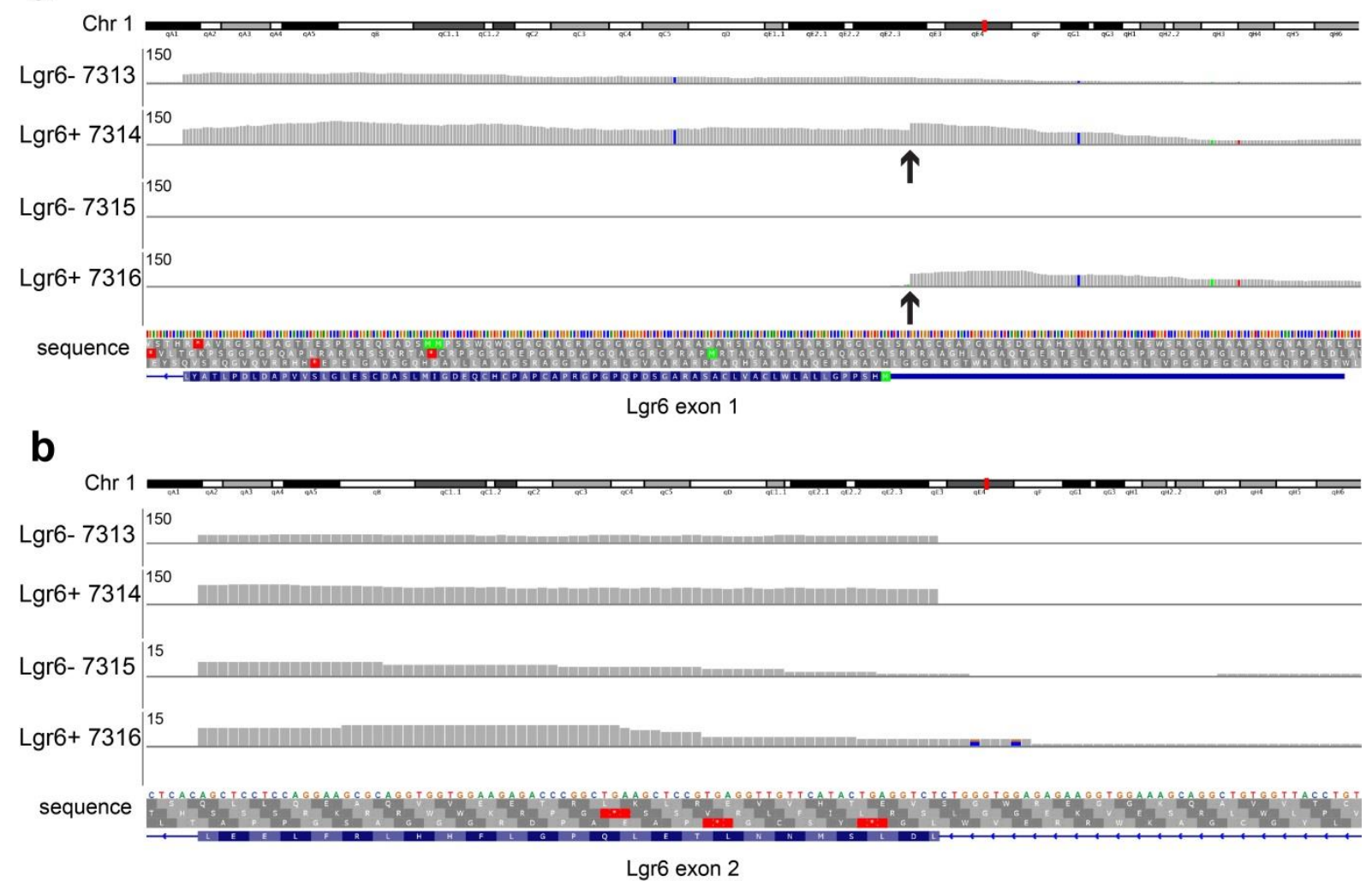

Figure S3. Lgr6 expression of exon 1 and exon 2 in a heterozygous and a homozygous sample. Screenshot from IG viewer of the base counts for Lgr6 exon 1 (a) and exon 2 (b) are depicted for epidermal basal cells (Lgr6-, odd samples) and for Lgr6+ stem cells (even samples). Samples 7313 and 7314 are heterozygous and 7315 and 7316 are homozygous transgenic. The arrow (a) shows the position were the cassette (EGFP-Ires-CreERT2) is inserted. This results in no transcription of exon 1 in the homozygous sample. In the homozygous sample transcription starts in close proximity to exon 2 (b). 


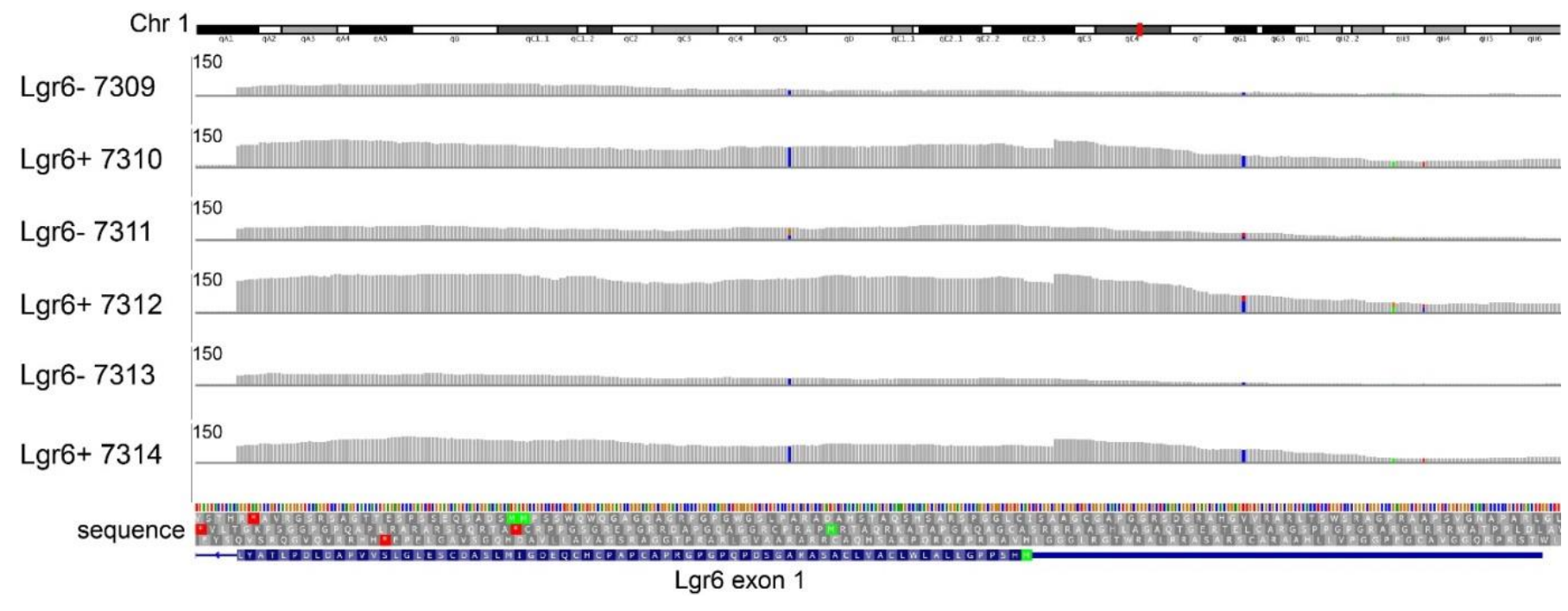

Figure S4. IGV visualization of reads aligned to Exon 1 of the Lgr6 gene for all heterozygous transgenic samples. Read count for Lgr6 exon 1 is shown (y-axis $=150$ ) for epidermal basal cells (Lgr6 ${ }^{-}$, odd samples) and Lgr6 $^{+}$stem cells (even samples). Lgr6 ${ }^{+}$stem cells express higher counts (also after normalization), however the Lgr6 $^{-}$samples are not completely negative for Lgr6.

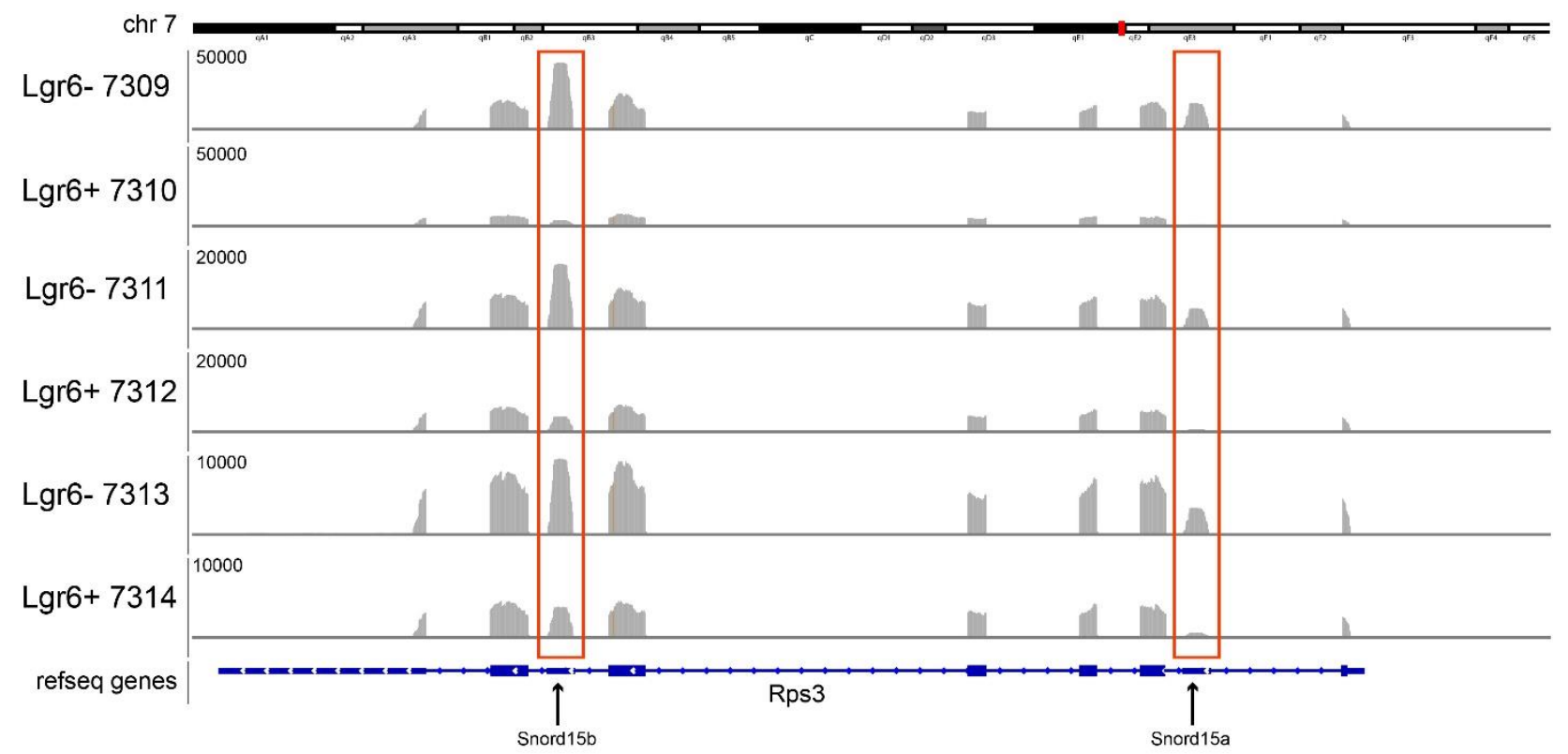

Figure S5. IGV screenshot of (m)RNA expression corresponding to the region encoding the Rps3 gene (including Snord15a and Snord15b) for all heterozygous transgenic samples. The counts for the Rps3 gene, Snord15a and Snord15b are represented (y-axis, values variate), for the epidermal basal cells (Lgr6 ${ }^{-}$, odd numbering) and for the Lgr6 $^{+}$stem cells (even numbering). The Lgr6 ${ }^{+}$cells show lower counts for Rps3 compared to the Lgr6 ${ }^{-}$ cells. Snord15a and Snord15b are even more prominently downregulated in Lgr6 ${ }^{+}$cells (see red boxes and arrows). 


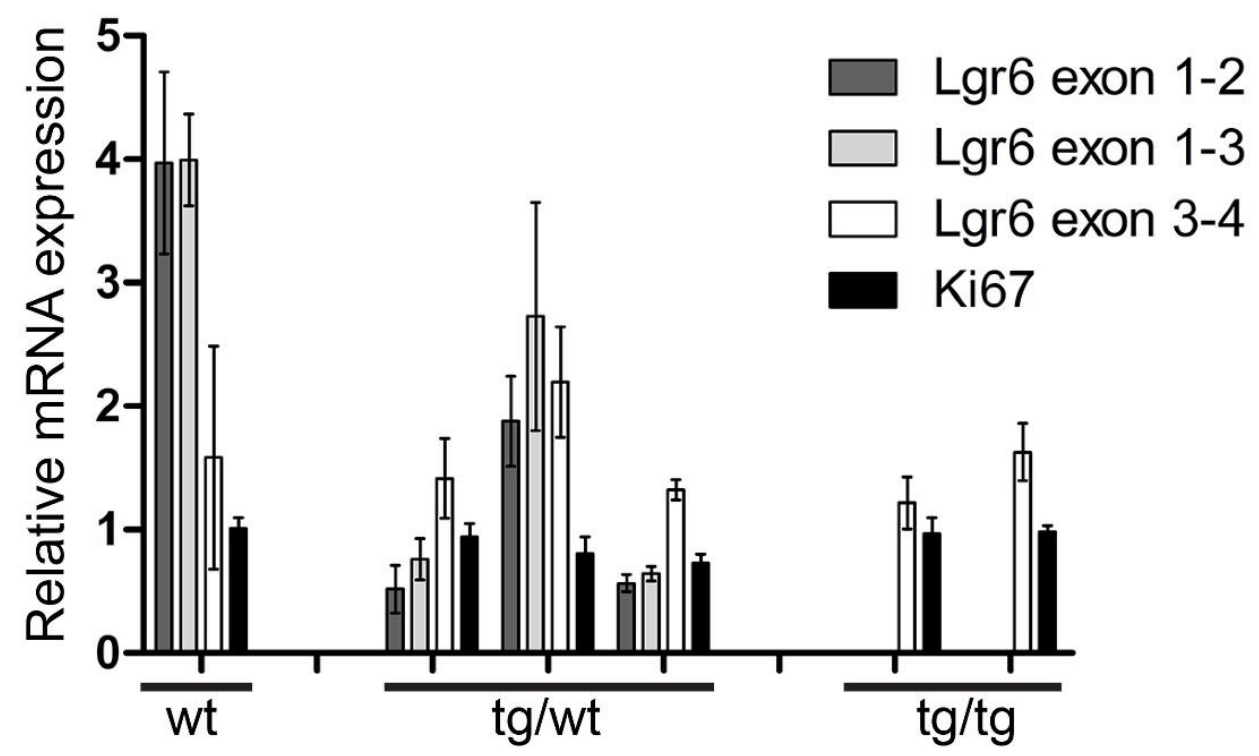

Figure S6. Relative mRNA expression of Lgr6 in hairless mice. Gene expression of Lgr6 and Ki67 was measured by qPCR and normalized against stably expressed reference genes (see Methods above and Supplementary table S3 for used primers). RNA was isolated from SKH-1 mice (wt), heterozygous Lgr6-EGFP-Ires-CreERT2/R26R-LacZ mice (Tg/wt) and homozygous Lgr6-EGFP-Ires-CreERT2/R26R-LacZ mice (Tg/Tg). Lgr6 gene expression was measured using different primer pairs. The primer pairs for exon 1-2 and exon 1-3 detect expression of the wt Lgr6 allele, the primers for exon 3-4 detect expression of the wt allele and the alternative transcript. In homozygous Lgr6-EGFP-Ires-CreERT2/R26R-LacZ mice expression of exon 1-2 and exon 1-3 is not detected at all, however expression of Lgr6 exon 3-4 was found. Error bars are SEMs. 
SUPPLEMENTARY INFORMATION Part 2

RNA-seq analysis identifies an Lgr6 isoform and gene expression pattern characterizing adult skin stem cells

GC van de Glind*1, AN Bastidas Torres*1, WH Zoutman ${ }^{1}$, SAJ van der Zeeuw ${ }^{2}$, SM Kielbasa $^{2}$, FR de Gruijl ${ }^{1}$ and CP Tensen ${ }^{\# 1}$

${ }^{1}$ Department of Dermatology, Leiden University Medical Center, Leiden, The Netherlands ${ }^{2}$ Department of Medical Statistics and Bioinformatics, Leiden University Medical Center, Leiden, The Netherlands

*Contributed equally to this study

Supplementary Table S1 All upregulated (a) and downregulated (b) genes in Lgr6 ${ }^{+}$basal epidermal keratinocytes (FDR $<0.05)$.

a. upregulated cells in Lgr6 $^{+}$cells

\begin{tabular}{|c|c|c|c|c|c|c|c|c|c|c|c|}
\hline genes & $\log \mathrm{FC}$ & $\log C P M$ & LR & PValue & FDR & S7309 & S7311 & S7313 & S7310 & S7312 & S7314 \\
\hline Igfbp3 & $1.7449 \mathrm{e}+00$ & 9.54690426 & 72.978 & $1.31 \mathrm{E}-17$ & $1.71 \mathrm{E}-14$ & 324.1 & 403.13 & 304 & 1305.4 & 1300.2 & 850. \\
\hline Adam8 & $1.2265 \mathrm{e}+00$ & 7.08857733 & 68.619 & 1.19E-16 & $1.31 \mathrm{E}-13$ & 83.817 & 77.296 & 83.216 & 214.35 & 196.17 & 161.2 \\
\hline Nat8l & $1.4536 \mathrm{e}+00$ & 5.08290211 & 64.432 & $9.99 \mathrm{E}-16$ & $9.08 \mathrm{E}-13$ & 14.311 & 21.148 & 19.067 & 42.709 & 52.784 & 53.03 \\
\hline Esr1 & $2.2224 \mathrm{e}+00$ & 3.66258455 & 62.661 & $2.46 \mathrm{E}-15$ & $2.07 \mathrm{E}-12$ & 3.1384 & 4.1264 & 6.3258 & 16.389 & 24.489 & 21.3 \\
\hline Skint7 & $1.1538 \mathrm{e}+00$ & 6.89963398 & 62.646 & 2.47E-15 & 2.07E-12 & 64.024 & 82.012 & 76.177 & 154.93 & 165.88 & 172.95 \\
\hline Crispld2 & $1.1386 \mathrm{e}+00$ & 5.18984284 & 61.572 & 4.27E-15 & 3.3E-12 & 21.258 & 22.99 & 24.145 & 53.043 & 48.977 & 48.1 \\
\hline Adamts14 & $1.1792 \mathrm{e}+00$ & 7.04431775 & 59.966 & $9.65 \mathrm{E}-15$ & $6.5 \mathrm{E}-12$ & 69.045 & 84.665 & 89.007 & 165.02 & 203.61 & 180. \\
\hline Ngfr & $1.4180 \mathrm{e}+00$ & 5.25109094 & 49.561 & $1.92 \mathrm{E}-12$ & $7.87 \mathrm{E}-10$ & 19.207 & 23.948 & 18.977 & 55.546 & 70.264 & 40.1 \\
\hline Irs1 & $1.7518 \mathrm{e}+00$ & 4.75758712 & 48.582 & 3.17E-12 & 1.23E-09 & 9.7082 & 17.463 & 9.9788 & 36.169 & 54.602 & \\
\hline Epb4.113 & $1.2735 \mathrm{e}+00$ & 4.92353235 & 48.021 & $4.22 \mathrm{E}-12$ & $1.55 \mathrm{E}-09$ & 16.655 & 19.6 & 16.928 & 45.292 & 49.583 & 33.5 \\
\hline Dmpk & $1.6867 \mathrm{e}+00$ & 4.08939534 & 46.435 & 9.47E-12 & 3.3E-09 & 6.4861 & 9.7265 & 8.0187 & 23.009 & 34.526 & 20.0 \\
\hline Dio2 & $1.3821 \mathrm{e}+00$ & 5.05977374 & 45.085 & 1.89E-11 & $6.16 \mathrm{E}-09$ & 15.525 & 16.211 & 23.878 & 40.045 & 44.304 & 59.872 \\
\hline Kitl & $1.9530 \mathrm{e}+00$ & 3.56224904 & 40.66 & $1.81 \mathrm{E}-10$ & $5.11 \mathrm{E}-08$ & 3.4313 & 5.7475 & 5.4349 & 25.432 & 18.431 & $12.0 s$ \\
\hline Limch1 & $1.1370 \mathrm{e}+00$ & 6.23916695 & 39.96 & $2.59 \mathrm{E}-10$ & 7.12E-08 & 54.023 & 35.443 & 51.943 & 121.34 & 89.214 & 100.72 \\
\hline Myh14 & $8.7376 \mathrm{e}-01$ & 6.19157637 & 39.737 & $2.91 \mathrm{E}-10$ & $7.78 \mathrm{E}-08$ & 57.705 & 48.632 & 48.023 & 92.684 & 92.33 & 98 \\
\hline Golga7b & $1.8483 e+00$ & 4.95680827 & 36.684 & $1.39 \mathrm{E}-09$ & $3.45 \mathrm{E}-07$ & 9.7919 & 18.79 & 11.939 & 41.094 & 74.244 & 30.2 \\
\hline Foxq1 & $1.2222 \mathrm{e}+00$ & 5.14092325 & 36.576 & 1.47E-09 & $3.57 \mathrm{E}-07$ & 18.705 & 24.169 & 20.581 & 38.511 & 65.332 & 44.0 \\
\hline Tspear & $1.6016 \mathrm{e}+00$ & 3.90722155 & 36.229 & $1.75 \mathrm{E}-09$ & 4.16E-07 & 6.0676 & 9.3581 & 6.8604 & 19.942 & 30.805 & 16.655 \\
\hline Ablim3 & $1.2686 \mathrm{e}+00$ & 3.97220759 & 35.692 & $2.31 \mathrm{E}-09$ & $5.25 \mathrm{E}-07$ & 9.2479 & 8.6949 & 9.5333 & 19.861 & 19.037 & 27.35 \\
\hline Gdpd2 & $8.9909 \mathrm{e}-01$ & 6.43633925 & 33.716 & $6.38 \mathrm{E}-09$ & $1.33 \mathrm{E}-06$ & 55.069 & 62.633 & 63.615 & 93.733 & 131.53 & 112.56 \\
\hline Abcc5 & $8.2111 \mathrm{e}-01$ & 8.15482089 & 32.282 & 1.33E-08 & $2.63 \mathrm{E}-06$ & 195.84 & 205.51 & 216.59 & 334.32 & 376.41 & 380.79 \\
\hline Npr3 & $1.5884 \mathrm{e}+00$ & 3.25043551 & 32.221 & $1.38 \mathrm{E}-08$ & $2.66 \mathrm{E}-06$ & 4.5193 & 6.1896 & 3.3857 & 15.501 & 16.095 & 10.95 \\
\hline Pls1 & $1.3763 e+00$ & 3.71877907 & 31.88 & $1.64 \mathrm{E}-08$ & $3.14 \mathrm{E}-06$ & 6.5279 & 7.2949 & 8.1078 & 20.91 & 22.325 & 13.412 \\
\hline Sox6 & $9.7095 \mathrm{e}-01$ & 6.96172020 & 31.497 & $2 \mathrm{E}-08$ & $3.76 \mathrm{E}-06$ & 70.008 & 82.159 & 100.59 & 142.17 & 154.98 & 197. \\
\hline Wnt7b & $7.8482 \mathrm{e}-01$ & 7.13482732 & 31.014 & $2.56 \mathrm{E}-08$ & $4.78 \mathrm{E}-06$ & 109.09 & 103.31 & 96.937 & 198.37 & 173.24 & 161. \\
\hline Ccdc80 & $1.4790 \mathrm{e}+00$ & 3.70009250 & 30.74 & 2.95E-08 & $5.45 \mathrm{E}-06$ & 8.3691 & 6.4843 & 5.4349 & 23.817 & 20.248 & 13.061 \\
\hline Col4a4 & $4.0657 e+00$ & 0.26775538 & 30.705 & $3 \mathrm{E}-08$ & $5.5 \mathrm{E}-06$ & 0.16738 & 0.073686 & 0.089096 & 4.1175 & 1.298 & 0.96426 \\
\hline
\end{tabular}




\begin{tabular}{|c|c|c|c|c|c|c|c|c|c|c|c|}
\hline Dgki & $1.4240 \mathrm{e}+00$ & 3.53588907 & 30.476 & $3.38 \mathrm{E}-08$ & $6.11 \mathrm{E}-06$ & 4.8123 & 8.1054 & 5.9694 & 17.439 & 19.383 & 13.5 \\
\hline Slc2a12 & $7.3833 \mathrm{e}-01$ & 7.03039310 & 29.801 & $4.79 \mathrm{E}-08$ & 8.19E-06 & 95.869 & 104.93 & 92.927 & 170.19 & 165.19 & \\
\hline Cpxm2 & $7.7072 \mathrm{e}-01$ & 6.28348319 & 29.56 & $5.42 \mathrm{E}-08$ & $9.2 \mathrm{E}-06$ & 61.681 & 53.496 & 57.2 & 109.96 & 91.032 & 93.35 \\
\hline Ttc9 & $1.1428 \mathrm{e}+00$ & 4.00585268 & 28.475 & $9.49 \mathrm{E}-08$ & $1.54 \mathrm{E}-05$ & 9.8756 & 12.011 & 7.9296 & 21.314 & 25.354 & 19.3 \\
\hline Slc4a8 & $1.0628 \mathrm{e}+00$ & 4.29885682 & 28.005 & $1.21 \mathrm{E}-07$ & $1.93 \mathrm{E}-05$ & 11.173 & 12.084 & 14.968 & 22.283 & 25.44 & 31.73 \\
\hline Cdh4 & $1.2123 e+00$ & 4.03202647 & 27.818 & $1.33 \mathrm{E}-07$ & $2.08 \mathrm{E}-05$ & 8.7039 & 9.2844 & 11.583 & 16.389 & 25.354 & 26.4 \\
\hline Col16a1 & $8.5712 \mathrm{e}-01$ & 8.92759674 & 27.778 & $1.36 \mathrm{E}-07$ & $2.11 \mathrm{E}-05$ & 298.69 & 346.84 & 393.72 & 607.85 & 691.91 & \\
\hline Lgr6 & $1.1048 \mathrm{e}+00$ & 6.69144306 & 27.321 & $1.72 \mathrm{E}-07$ & $2.61 \mathrm{E}-05$ & 45.11 & 85.254 & 66.555 & 117.07 & 171.94 & 133.8 \\
\hline Phldb1 & $8.4835 \mathrm{e}-01$ & 7.33632062 & 27.085 & $1.95 \mathrm{E}-07$ & $2.93 \mathrm{E}-05$ & 108.8 & 125.56 & 111.73 & 218.23 & 240.47 & 164. \\
\hline Ripply3 & $1.2454 \mathrm{e}+00$ & 3.30258336 & 26.827 & $2.23 \mathrm{E}-07$ & $3.3 \mathrm{E}-05$ & 5.5655 & 5.8212 & 6.0585 & 14.048 & 15.922 & 11.308 \\
\hline Man1c1 & $1.1500 \mathrm{e}+00$ & 5.94092811 & 26.389 & $2.79 \mathrm{E}-07$ & $3.99 \mathrm{E}-05$ & 23.978 & 44.211 & 46.508 & 65.476 & 97.608 & 90.5 \\
\hline Slc16a9 & $8.4731 \mathrm{e}-01$ & 5.68169457 & 26.164 & $3.14 \mathrm{E}-07$ & 4.43E-05 & 37.368 & 39.495 & 32.876 & 77.425 & 68.187 & 51.9 \\
\hline Ror1 & $1.3467 \mathrm{e}+00$ & 3.85508008 & 25.794 & $3.8 \mathrm{E}-07$ & $5.29 \mathrm{E}-05$ & 7.2393 & 8.4738 & 8.7314 & 21.556 & 27.431 & 12.9 \\
\hline Col4a2 & $1.7095 \mathrm{e}+00$ & 4.89805970 & 25.041 & $5.61 \mathrm{E}-07$ & $7.56 \mathrm{E}-05$ & 14.813 & 18.421 & 8.4641 & 56.595 & 59.361 & 20.7 \\
\hline Mmp28 & $6.7536 \mathrm{e}-01$ & 6.59461898 & 24.832 & $6.26 \mathrm{E}-07$ & 8.17E-05 & 66.2 & 75.749 & 81.345 & 115.29 & 119.33 & 121. \\
\hline Il12rb2 & $1.3919 e+00$ & 5.55491878 & 24.352 & $8.03 \mathrm{E}-07$ & 0.000102 & 19.96 & 33.527 & 24.323 & 35.2 & 83.849 & 84.9 \\
\hline Abca4 & $1.8840 \mathrm{e}+00$ & 1.19371517 & 24.302 & $8.24 \mathrm{E}-07$ & 0.000104 & 0.83691 & 1.1053 & 0.89096 & 3.5523 & 3.8939 & \\
\hline II20ra & $7.3658 \mathrm{e}-01$ & 7.69178939 & 23.334 & $1.36 \mathrm{E}-06$ & 0.000165 & 137.21 & 168.52 & 159.48 & 224.85 & 284.34 & 265. \\
\hline Wnk2 & $7.5118 \mathrm{e}-01$ & 6.83668084 & 23.206 & $1.46 \mathrm{E}-06$ & 0.000175 & 80.093 & 95.644 & 79.652 & 120.46 & 165.36 & 144 \\
\hline Sntb2 & $6.8741 \mathrm{e}-01$ & 5.81609181 & 22.999 & $1.62 \mathrm{E}-06$ & 0.000192 & 39.879 & 43.548 & 45.974 & 61.036 & 70.697 & 76.3 \\
\hline Ccr4 & $9.9612 \mathrm{e}-01$ & 3.66020631 & 22.93 & $1.68 \mathrm{E}-06$ & 0.000198 & 9.1642 & 8.0317 & 7.8405 & 16.954 & 17.133 & 16.1 \\
\hline Crlf1 & $1.0861 \mathrm{e}+00$ & 3.49677431 & 22.813 & $1.79 \mathrm{E}-06$ & 0.00021 & 6.4442 & 6.558 & 8.6423 & 14.532 & 14.537 & \\
\hline Nrp1 & $2.2311 \mathrm{e}+00$ & 0.64570913 & 22.781 & $1.82 \mathrm{E}-06$ & 0.000211 & 0.66953 & 0.36843 & 0.44548 & 2.9065 & 1.5576 & \\
\hline Xkr4 & $1.9450 \mathrm{e}+00$ & 1.30727523 & 22.72 & $1.87 \mathrm{E}-06$ & 0.000216 & 0.79507 & 1.5474 & 0.62367 & 4.1982 & 3.8939 & 3.33 \\
\hline Lifr & $7.1906 \mathrm{e}-01$ & 7.10811403 & 22.677 & $1.92 \mathrm{E}-06$ & 0.00022 & 113.74 & 104.93 & 93.729 & 183.11 & 144.34 & 187. \\
\hline Hsd3b7 & $7.5553 \mathrm{e}-01$ & 4.97919105 & 22.507 & $2.09 \mathrm{E}-06$ & 0.000236 & 20.672 & 24.906 & 24.769 & 36.573 & 39.286 & 42.5 \\
\hline Cysltr1 & $8.4317 \mathrm{e}-01$ & 4.84837245 & 22.456 & $2.15 \mathrm{E}-06$ & 0.000241 & 19.667 & 22.843 & 19.156 & 39.076 & 41.708 & 29.8 \\
\hline Wnt16 & $1.0968 \mathrm{e}+00$ & 3.63043845 & 22.144 & $2.53 \mathrm{E}-06$ & 0.000276 & 8.8713 & 7.2949 & 7.2168 & 20.103 & 16.614 & 13.5 \\
\hline Antxr1 & $6.9221 \mathrm{e}-01$ & 8.14070762 & 22.14 & $2.53 \mathrm{E}-06$ & 0.000276 & 203.62 & 219.44 & 224.17 & 309.94 & 364.39 & 371. \\
\hline Frmd4a & $6.1125 \mathrm{e}-01$ & 6.15764326 & 22.08 & $2.61 \mathrm{E}-06$ & 0.000279 & 57.831 & 56.591 & 54.705 & 83.076 & 89.301 & \\
\hline Phospho1 & $7.3031 \mathrm{e}-01$ & 5.08024485 & 22.077 & $2.62 \mathrm{E}-06$ & 0.000279 & 23.266 & 27.632 & 25.303 & 37.38 & 44.045 & 44.7 \\
\hline Zfp652 & $7.0529 \mathrm{e}-01$ & 5.92200264 & 22.077 & $2.62 \mathrm{E}-06$ & 0.000279 & 40.256 & 47.601 & 50.428 & 64.507 & 75.715 & 84.7 \\
\hline Ackr2 & $9.7986 \mathrm{e}-01$ & 3.63668600 & 21.536 & $3.47 \mathrm{E}-06$ & 0.000363 & 7.867 & 8.5475 & 8.5532 & 17.842 & 14.883 & 16.3 \\
\hline Igfbp7 & $9.7521 \mathrm{e}-01$ & 4.36109562 & 21.331 & $3.86 \mathrm{E}-06$ & 0.000401 & 10.629 & 12.969 & 18.087 & 30.679 & 24.402 & 26.1 \\
\hline Csrp2 & $1.3611 \mathrm{e}+00$ & 2.97995669 & 20.822 & $5.04 \mathrm{E}-06$ & 0.000518 & 3.0547 & 4.0527 & 6.2367 & 10.011 & 12.547 & 11.1 \\
\hline Znrf3 & $6.1328 \mathrm{e}-01$ & 6.42678877 & 20.763 & $5.2 \mathrm{E}-06$ & 0.000532 & 65.991 & 69.412 & 68.426 & 95.187 & 105.05 & 11 \\
\hline Camkk1 & $6.1839 \mathrm{e}-01$ & 6.49327360 & 20.598 & $5.66 \mathrm{E}-06$ & 0.000574 & 74.192 & 63.443 & 75.286 & 108.18 & 102.89 & 115. \\
\hline Shisa2 & $3.2563 e+00$ & -0.51527234 & 20.527 & $5.88 \mathrm{E}-06$ & 0.000593 & 0.041846 & 0.22106 & 0.089096 & 1.6147 & 1.1249 & 0.525 \\
\hline Ikbke & $5.9542 \mathrm{e}-01$ & 6.51311568 & 20.465 & $6.07 \mathrm{E}-06$ & 0.00061 & 69.506 & 74.349 & 74.217 & 101.65 & 111.89 & \\
\hline Wipf3 & $1.4521 \mathrm{e}+00$ & 2.15401507 & 20.153 & 7.15E-06 & 0.00069 & 2.2597 & 2.3579 & 2.4056 & 6.1359 & 5.1919 & 7.88 \\
\hline Phka1 & $9.6263 \mathrm{e}-01$ & 3.93234595 & 20.14 & $7.2 \mathrm{E}-06$ & 0.00069 & 8.2018 & 11.2 & 11.672 & 17.52 & 22.152 & 20.4 \\
\hline Slc24a3 & $6.1925 \mathrm{e}-01$ & 6.75810442 & 20.138 & $7.21 \mathrm{E}-06$ & 0.00069 & 76.285 & 87.023 & 92.749 & 126.51 & 127.72 & 138. \\
\hline Col7a1 & $8.5312 \mathrm{e}-01$ & 10.54820699 & 20.052 & 7.54E-06 & 0.000712 & 918.05 & 1128.3 & 1155 & 1852.8 & 2286.3 & 164 \\
\hline 4930412013Rik & $6.9537 \mathrm{e}-01$ & 5.13390235 & 19.979 & $7.83 \mathrm{E}-06$ & 0.000737 & 25.777 & 27.632 & 26.818 & 38.107 & 49.669 & 42.0 \\
\hline Adrb1 & $1.4360 \mathrm{e}+00$ & 3.65018024 & 19.498 & $1.01 \mathrm{E}-05$ & 0.000927 & 4.9378 & 9.7265 & 5.6131 & 16.631 & 26.306 & 11.7 \\
\hline
\end{tabular}




\begin{tabular}{|c|c|c|c|c|c|c|c|c|c|c|c|}
\hline Cecr2 & $5.6707 \mathrm{e}-01$ & 6.50847162 & 19.432 & $1.04 \mathrm{E}-05$ & 0.000955 & 69.757 & 75.896 & 74.306 & 108.91 & 110.24 & 106.51 \\
\hline Mcc & $6.5736 \mathrm{e}-01$ & 7.59899258 & 19.404 & $1.06 \mathrm{E}-05$ & 0.000965 & 167.76 & 156.21 & 126.87 & 237.28 & 242.9 & 231.51 \\
\hline Ctsl & $7.8874 \mathrm{e}-01$ & 5.51114432 & 19.249 & $1.15 \mathrm{E}-05$ & 0.001029 & 35.36 & 31.98 & 32.698 & 73.792 & 54.602 & 44.53 \\
\hline Fras1 & $1.1795 e+00$ & 3.90031323 & 19.231 & $1.16 \mathrm{E}-05$ & 0.001029 & 7.7415 & 6.6317 & 13.097 & 16.631 & 19.297 & \\
\hline Golm1 & $1.5419 e+00$ & 2.71877841 & 18.882 & $1.39 \mathrm{E}-05$ & 0.00121 & 2.9292 & 1.9158 & 5.2567 & 7.912 & 9.0859 & 12.09 \\
\hline Zfp503 & $8.2471 \mathrm{e}-01$ & 5.44986683 & 18.865 & 1.4E-05 & 0.001216 & 23.183 & 37.58 & 33.946 & 52.074 & 65.678 & 49.3 \\
\hline Fuk & $6.8438 \mathrm{e}-01$ & 5.02160783 & 18.415 & $1.78 \mathrm{E}-05$ & 0.001484 & 22.471 & 25.053 & 27.174 & 44.808 & 37.814 & 36.992 \\
\hline Col4a6 & $6.8641 \mathrm{e}-01$ & 7.81764797 & 18.362 & $1.83 \mathrm{E}-05$ & 0.001508 & 179.56 & 181.27 & 157.61 & 315.43 & 293.34 & 225. \\
\hline Lonrf3 & $9.4918 \mathrm{e}-01$ & 4.70171198 & 18.325 & $1.86 \mathrm{E}-05$ & 0.001522 & 13.935 & 16.506 & 22.898 & 27.288 & 32.276 & 42.8 \\
\hline Unc5b & $5.9891 \mathrm{e}-01$ & 6.02849452 & 18.287 & $1.9 \mathrm{E}-05$ & 0.001544 & 45.821 & 51.506 & 58.447 & 82.188 & 75.369 & 77.7 \\
\hline Parpbp & $6.9086 \mathrm{e}-01$ & 4.56167220 & 18.24 & $1.95 \mathrm{E}-05$ & 0.001577 & 17.324 & 18.937 & 17.73 & 28.742 & 29.767 & 28.5 \\
\hline Slc27a3 & $7.5655 \mathrm{e}-01$ & 8.08341986 & 18.221 & 1.97E-05 & 0.00158 & 163.83 & 233.51 & 207.77 & 350.79 & 391.47 & 279. \\
\hline Gpr64 & $8.2704 \mathrm{e}-01$ & 4.16405476 & 18.016 & $2.19 \mathrm{E}-05$ & 0.001733 & 14.604 & 13.411 & 10.335 & 24.705 & 22.239 & 21.5 \\
\hline Mef2a & $6.5677 \mathrm{e}-01$ & 7.21137745 & 17.946 & $2.27 \mathrm{E}-05$ & 0.001777 & 97.626 & 118.93 & 128.57 & 161.31 & 178 & 204.25 \\
\hline Fam124b & $3.0533 e+00$ & -0.52336618 & 17.914 & $2.31 \mathrm{E}-05$ & 0.001801 & 0.12554 & 0.22106 & 0 & 0.88809 & 1.8172 & 0.525 \\
\hline Slc6a2 & $5.7604 \mathrm{e}-01$ & 6.62445403 & 17.811 & $2.44 \mathrm{E}-05$ & 0.001888 & 73.104 & 77.444 & 87.047 & 108.67 & 118.12 & 127. \\
\hline Trim47 & $6.0338 \mathrm{e}-01$ & 6.58076592 & 17.747 & $2.52 \mathrm{E}-05$ & 0.001938 & 66.158 & 77.886 & 83.929 & 108.02 & 110.76 & 127. \\
\hline Pbxip1 & $6.9589 \mathrm{e}-01$ & 4.36990954 & 17.559 & $2.79 \mathrm{E}-05$ & 0.002116 & 16.194 & 14.59 & 16.305 & 25.997 & 25.787 & 24.5 \\
\hline Col4a3 & $2.8084 \mathrm{e}+00$ & -0.05119185 & 17.455 & $2.94 \mathrm{E}-05$ & 0.002218 & 0.33477 & 0.14737 & 0.089096 & 2.5835 & 1.471 & 0.525 \\
\hline Wnt10b & $1.0340 \mathrm{e}+00$ & 3.89566953 & 17.437 & $2.97 \mathrm{E}-05$ & 0.002232 & 10.671 & 10.316 & 8.0187 & 26.4 & 19.037 & \\
\hline Cong2 & $6.5757 \mathrm{e}-01$ & 6.72863785 & 17.418 & $3 E-05$ & 0.002245 & 72.184 & 86.802 & 87.849 & 134.18 & 148.49 & 106. \\
\hline Cgnl1 & $2.5474 \mathrm{e}+00$ & 0.26010348 & 17.386 & $3.05 \mathrm{E}-05$ & 0.002276 & 0.37661 & 0.44211 & 0.089096 & 2.9872 & 1.9902 & 0.701 \\
\hline Lama3 & $6.7165 \mathrm{e}-01$ & 9.52688963 & 17.301 & $3.19 \mathrm{E}-05$ & 0.002372 & 555.04 & 523.9 & 627.95 & 915.05 & 835.99 & 967. \\
\hline Afap1l1 & $1.2588 \mathrm{e}+00$ & 2.72702413 & 17.271 & $3.24 \mathrm{E}-05$ & 0.0024 & 2.8455 & 3.4632 & 5.4349 & 8.5579 & 9.7781 & 9.2919 \\
\hline |l22ra2 & $8.4908 \mathrm{e}-01$ & 5.15336899 & 17.226 & $3.32 \mathrm{E}-05$ & 0.00245 & 22.053 & 30.874 & 23.165 & 49.329 & 55.034 & 32.52 \\
\hline Psd3 & $5.7179 \mathrm{e}-01$ & 6.22525551 & 17.178 & 3.4E-05 & 0.002502 & 61.597 & 59.096 & 59.516 & 82.592 & 85.58 & 99.8 \\
\hline $\mathrm{Kdm} 5 \mathrm{~d}$ & $6.1870 \mathrm{e}-01$ & 5.61600627 & 17.122 & $3.51 \mathrm{E}-05$ & 0.002561 & 33.853 & 44.506 & 37.599 & 59.825 & 61.957 & 55.92 \\
\hline Trpc1 & $1.1107 \mathrm{e}+00$ & 2.81462252 & 17.116 & $3.52 \mathrm{E}-05$ & 0.002561 & 3.6406 & 4.4211 & 5.2567 & 9.3653 & 8.9128 & 10.1 \\
\hline Trim7 & 5.3833e-01 & 6.34188361 & 17.115 & $3.52 \mathrm{E}-05$ & 0.002561 & 67.079 & 66.022 & 65.04 & 96.478 & 92.676 & 98.7 \\
\hline Ascl2 & $1.0205 e+00$ & 3.98287384 & 17.09 & 3.57E-05 & 0.002586 & 7.6159 & 12.748 & 10.959 & 21.718 & 25.613 & 15.779 \\
\hline Abcg1 & $5.7557 \mathrm{e}-01$ & 5.63826626 & 16.992 & $3.75 \mathrm{E}-05$ & 0.002704 & 36.615 & 40.527 & 42.766 & 55.788 & 60.572 & 61.9 \\
\hline $\mathrm{Clu}$ & $9.0713 \mathrm{e}-01$ & 4.68481688 & 16.961 & $3.81 \mathrm{E}-05$ & 0.002738 & 21.676 & 18.79 & 12.741 & 39.722 & 34.699 & 25.947 \\
\hline Serpinb2 & $6.7229 \mathrm{e}-01$ & 8.71554538 & 16.855 & 4.03E-05 & 0.002885 & 290.28 & 300.78 & 381.42 & 579.11 & 478.52 & 491 \\
\hline Adck3 & 6.6179e-01 & 4.89576341 & 16.793 & 4.17E-05 & 0.002971 & 21.592 & 23.285 & 24.145 & 34.151 & 34.007 & 40.849 \\
\hline 1133 & $7.2241 \mathrm{e}-01$ & 6.37327574 & 16.775 & $4.21 \mathrm{E}-05$ & 0.00299 & 70.384 & 52.022 & 64.951 & 129.66 & 89.993 & 89.6 \\
\hline Ttc3 & $6.3101 \mathrm{e}-01$ & 8.16291162 & 16.663 & 4.46E-05 & 0.00315 & 215.25 & 212.88 & 246.44 & 323.34 & 320.34 & 400. \\
\hline Pgm5 & $1.6585 \mathrm{e}+00$ & 1.68268612 & 16.627 & $4.55 \mathrm{E}-05$ & 0.003189 & 1.0043 & 1.9158 & 1.6928 & 2.9872 & 5.538 & 5.7855 \\
\hline Ccdc17 & $9.3177 \mathrm{e}-01$ & 3.56787295 & 16.604 & 4.61E-05 & 0.003218 & 7.6578 & 9.6528 & 6.9495 & 13.241 & 16.355 & 16.7 \\
\hline Plcxd2 & $7.7138 \mathrm{e}-01$ & 4.10812856 & 16.552 & 4.73E-05 & 0.003295 & 13.809 & 12.821 & 11.226 & 21.072 & 23.71 & 20.162 \\
\hline Papln & $6.2690 \mathrm{e}-01$ & 6.07116605 & 16.482 & $4.91 \mathrm{E}-05$ & 0.003407 & 44.9 & 56.001 & 57.645 & 68.948 & 88.349 & 87.0 \\
\hline Pdgfb & $7.3172 \mathrm{e}-01$ & 5.42533089 & 16.419 & $5.08 \mathrm{E}-05$ & 0.003512 & 23.894 & 39.127 & 33.946 & 52.881 & 58.063 & 49. \\
\hline Rapgef4 & 8.6696e-01 & 4.25236975 & 16.379 & 5.19E-05 & 0.003574 & 10.001 & 15.474 & 15.057 & 28.661 & 21.806 & 22.8 \\
\hline Aknad1 & $9.5365 \mathrm{e}-01$ & 3.83744680 & 16.316 & 5.36E-05 & 0.003683 & 8.0762 & 8.916 & 12.206 & 22.283 & 14.883 & 18.935 \\
\hline Enthd2 & $5.7019 \mathrm{e}-01$ & 5.49777193 & 16.265 & $5.51 \mathrm{E}-05$ & 0.003772 & 33.853 & 38.906 & 36.173 & 49.733 & 56.159 & 55.6 \\
\hline Cbr2 & $6.8320 \mathrm{e}-01$ & 4.91730926 & 16.075 & $6.09 \mathrm{E}-05$ & 0.004141 & 22.053 & 23.211 & 24.145 & 36.734 & 43.439 & 31.1 \\
\hline
\end{tabular}




\begin{tabular}{|c|c|c|c|c|c|c|c|c|c|c|c|}
\hline Cxcl14 & $7.9693 \mathrm{e}-01$ & 6.48567631 & 16.002 & $6.33 \mathrm{E}-05$ & 0.004291 & 62.015 & 79.728 & 54.438 & 124.57 & 136.89 & 79. \\
\hline Th & $2.9242 \mathrm{e}+00$ & -1.0237201 & 15.857 & $6.83 \mathrm{E}-05$ & 0.004573 & 0.083691 & 0.073686 & 0.089096 & 0.40368 & 0.86532 & 0.788 \\
\hline Bcar3 & $6.1300 \mathrm{e}-01$ & 5.24545962 & 15.81 & 7E-05 & 0.004643 & 31.635 & 29.916 & 28.065 & 51.186 & 46.208 & 39.8 \\
\hline Cpe & 6.6499e-01 & 6.74590095 & 15.743 & $7.25 \mathrm{E}-05$ & 0.004779 & 68.418 & 74.201 & 106.56 & 129.74 & 132.83 & 131. \\
\hline Sema3e & $2.9131 \mathrm{e}+00$ & 0.51471081 & 15.662 & $7.57 \mathrm{E}-05$ & 0.004972 & 0.41846 & 0.36843 & 0.089096 & 4.7634 & 1.9037 & 0.43 \\
\hline Grem1 & $3.0035 \mathrm{e}+00$ & -0.46575827 & 15.643 & 7.65E-05 & 0.004979 & 0.25107 & 0.073686 & 0 & 1.7762 & 1.2114 & $0.35 c$ \\
\hline Mrgprd & $5.2808 \mathrm{e}+00$ & -1.77957399 & 15.643 & $7.65 \mathrm{E}-05$ & 0.004979 & 0 & 0 & 0 & 0.48441 & 0.086532 & 0.43 \\
\hline Abcb1b & $6.7692 \mathrm{e}-01$ & 5.73660233 & 15.5 & $8.25 \mathrm{E}-05$ & 0.005335 & 36.029 & 34.19 & 52.923 & 61.682 & 64.034 & 70.5 \\
\hline Mafa & $1.5517 \mathrm{e}+00$ & 2.54288016 & 15.47 & $8.38 \mathrm{E}-05$ & 0.005402 & 1.4646 & 3.3159 & 4.1875 & 7.6698 & 11.682 & 6.39 \\
\hline Als2cl & $6.6757 \mathrm{e}-01$ & 8.17556771 & 15.216 & 9.59E-05 & 0.006035 & 199.35 & 211.18 & 259.63 & 300.58 & 342.58 & \\
\hline Npr1 & $1.6176 \mathrm{e}+00$ & 0.86709864 & 15.215 & 9.59E-05 & 0.006035 & 0.96245 & 0.73686 & 0.80187 & 3.3101 & 2.5094 & 2.01 \\
\hline Raph1 & 5.4849e-01 & 6.64636641 & 15.179 & $9.78 \mathrm{E}-05$ & 0.006115 & 81.85 & 78.549 & 83.394 & 110.2 & 108.86 & \\
\hline Gpr116 & $2.0726 \mathrm{e}+00$ & 0.18133361 & 15.066 & 0.000104 & 0.006437 & 0.54399 & 0.22106 & 0.35638 & 2.3413 & 1.8172 & 0.87 \\
\hline Arhgef28 & $5.2405 \mathrm{e}-01$ & 5.95698395 & 15.065 & 0.000104 & 0.006437 & 52.516 & 48.19 & 51.854 & 76.375 & 65.159 & \\
\hline Ccna1 & $2.2580 \mathrm{e}+00$ & -0.48461602 & 15.042 & 0.000105 & 0.006496 & 0.20923 & 0.22106 & 0.17819 & 1.3725 & 0.77879 & \\
\hline Kcnip3 & $9.4433 \mathrm{e}-01$ & 3.21716917 & 15.019 & 0.000106 & 0.006539 & 5.1889 & 7.2212 & 6.5931 & 12.352 & 12.807 & 11.1 \\
\hline Rhbdl1 & $9.1382 \mathrm{e}-01$ & 3.52495792 & 14.991 & 0.000108 & 0.006597 & 7.1556 & 9.0633 & 7.5732 & 13.16 & 18.345 & 13.2 \\
\hline Hs3st3a1 & $2.5340 \mathrm{e}+00$ & -0.330613 & 14.959 & 0.00011 & 0.006691 & 0.083691 & 0.073686 & 0.53458 & 0.72662 & 1.471 & 1.40 \\
\hline Ankrd6 & $1.3524 \mathrm{e}+00$ & 2.50861642 & 14.76 & 0.000122 & 0.007287 & 2.7618 & 2.9474 & 3.8311 & 5.8129 & 6.4034 & \\
\hline Dnah8 & $7.2306 \mathrm{e}-01$ & 4.77615668 & 14.739 & 0.000123 & 0.007346 & 19.416 & 19.306 & 23.165 & 28.661 & 30.892 & \\
\hline Fgfr3 & $6.5852 \mathrm{e}-01$ & 9.13775294 & 14.73 & 0.000124 & 0.00736 & 357.74 & 447.71 & 505.35 & 634.25 & 676.85 & \\
\hline Rgs11 & $1.0329 \mathrm{e}+00$ & 3.23480725 & 14.661 & 0.000129 & 0.00755 & 5.6492 & 4.7159 & 8.1078 & 10.98 & 11.509 & 15.0 \\
\hline Mfsd7b & $5.4072 \mathrm{e}-01$ & 5.85321068 & 14.609 & 0.000132 & 0.007718 & 41.846 & 48.632 & 50.785 & 76.133 & 62.043 & 66.7 \\
\hline 2010016I18Rik & $1.2267 \mathrm{e}+00$ & 2.45777619 & 14.552 & 0.000136 & 0.007899 & 2.3434 & 4.3474 & 3.1184 & 6.6203 & 8.3071 & 7.80 \\
\hline Wnt3a & $6.8910 \mathrm{e}-01$ & 5.27551980 & 14.513 & 0.000139 & 0.008007 & 23.266 & 33.895 & 31.807 & 39.399 & 51.919 & 51.6 \\
\hline Slc12a4 & $5.1466 \mathrm{e}-01$ & 7.41082967 & 14.498 & 0.00014 & 0.00805 & 139.93 & 135.88 & 144.34 & 200.87 & 191.75 & \\
\hline Espl1 & $5.3674 \mathrm{e}-01$ & 5.49909252 & 14.431 & 0.000145 & 0.008319 & 36.908 & 35.59 & 37.955 & 49.248 & 52.092 & 58.9 \\
\hline KIf12 & $1.3271 \mathrm{e}+00$ & 2.23905763 & 14.396 & 0.000148 & 0.008408 & 2.8037 & 3.0948 & 1.9601 & 6.1359 & 4.8458 & \\
\hline Ddx3y & $6.6155 \mathrm{e}-01$ & 6.95732646 & 14.38 & 0.000149 & 0.008456 & 72.519 & 105.81 & 110.57 & 161.63 & 163.63 & \\
\hline Adcy6 & 5.3063e-01 & 5.67828870 & 14.326 & 0.000154 & 0.008675 & 37.787 & 42.738 & 45.083 & 58.452 & 57.89 & \\
\hline Fgfr2 & $5.8601 \mathrm{e}-01$ & 9.18049115 & 14.212 & 0.000163 & 0.009095 & 425.28 & 503.57 & 462.94 & 651.61 & 743.4 & \\
\hline Itga2 & $5.2405 \mathrm{e}-01$ & 5.88092573 & 14.204 & 0.000164 & 0.00911 & 45.905 & 46.938 & 52.032 & 67.495 & 62.476 & 78.1 \\
\hline Slc16a3 & $1.2784 \mathrm{e}+00$ & 3.60007701 & 14.171 & 0.000167 & 0.009205 & 4.1427 & 5.8948 & 11.315 & 16.712 & 12.201 & 22.2 \\
\hline Ccdc3 & $7.0845 \mathrm{e}-01$ & 7.83788994 & 14.165 & 0.000167 & 0.009205 & 183.08 & 193.2 & 144.51 & 334.65 & 308.05 & \\
\hline Lamc2 & $5.5791 \mathrm{e}-01$ & 8.90939350 & 14.117 & 0.000172 & 0.009397 & 387.87 & 361.06 & 417.86 & 579.35 & 548.61 & \\
\hline Gas213 & $6.3623 \mathrm{e}-01$ & 5.38942546 & 14.111 & 0.000172 & 0.009397 & 36.699 & 26.379 & 35.015 & 46.746 & 46.727 & 59.2 \\
\hline Tgm7 & $1.5354 \mathrm{e}+00$ & 2.44061092 & 14.04 & 0.000179 & 0.009734 & 2.4689 & 4.0527 & 1.6928 & 5.3285 & 12.461 & \\
\hline Zfr2 & $7.2175 \mathrm{e}-01$ & 4.05350636 & 14.029 & 0.00018 & 0.009746 & 10.964 & 13.779 & 12.741 & 18.488 & 22.325 & 20.7 \\
\hline Ttc14 & $5.6735 \mathrm{e}-01$ & 7.28176623 & 14.027 & 0.00018 & 0.009746 & 112.77 & 141.11 & 122.15 & 160.82 & 204.39 & \\
\hline Axin2 & $5.2398 \mathrm{e}-01$ & 7.07264361 & 13.978 & 0.000185 & 0.009933 & 107.33 & 115.61 & 108.07 & 138.86 & 169.17 & 167 \\
\hline Foxp1 & $7.0136 \mathrm{e}-01$ & 5.18547545 & 13.928 & 0.00019 & 0.010166 & 23.475 & 24.758 & 34.926 & 38.914 & 41.622 & 54.1 \\
\hline DII1 & $5.3065 \mathrm{e}-01$ & 7.17675454 & 13.91 & 0.000192 & 0.010166 & 113.49 & 114.07 & 127.41 & 188.11 & 151.69 & 172. \\
\hline Cd55 & $5.2636 \mathrm{e}-01$ & 5.97262576 & 13.852 & 0.000198 & 0.010404 & 50.466 & 52.612 & 51.052 & 69.028 & 67.235 & 85.7 \\
\hline Plcb4 & $7.8408 \mathrm{e}-01$ & 3.74063143 & 13.81 & 0.000202 & 0.010611 & 11.173 & 9.9476 & 7.9296 & 17.6 & 16.268 & 16.5 \\
\hline Gm9926 & $4.7202 \mathrm{e}+00$ & -2.05079697 & 13.798 & 0.000204 & 0.010644 & 0 & 0 & 0 & 0.24221 & 0.17306 & 0.262 \\
\hline
\end{tabular}




\begin{tabular}{|c|c|c|c|c|c|c|c|c|c|c|c|}
\hline Eif4e3 & $5.6062 \mathrm{e}-01$ & 5.05229115 & 13.795 & 0.000204 & 0.010644 & 24.145 & 27.337 & 28.867 & 37.945 & 41.622 & 38.57 \\
\hline Ptch2 & $1.9268 \mathrm{e}+00$ & -0.09561434 & 13.748 & 0.000209 & 0.010859 & 0.41846 & 0.22106 & 0.35638 & 1.534 & 0.86532 & 1.5779 \\
\hline Incenp & $5.7620 \mathrm{e}-01$ & 6.08107320 & 13.706 & 0.000214 & 0.010962 & 54.567 & 46.201 & 62.1 & 69.271 & 82.811 & 90.6 \\
\hline Sema3f & $5.2150 \mathrm{e}-01$ & 7.80422310 & 13.692 & 0.000215 & 0.010973 & 185.46 & 196.89 & 167.95 & 270.87 & 268.51 & 250.7 \\
\hline TtII7 & $7.4834 \mathrm{e}-01$ & 3.96878696 & 13.609 & 0.000225 & 0.011357 & 9.499 & 12.084 & 13.454 & 19.376 & 18.777 & 20.24 \\
\hline Anpep & $1.2613 \mathrm{e}+00$ & 3.37543151 & 13.571 & 0.00023 & 0.011506 & 6.3187 & 8.9896 & 2.762 & 12.837 & 18.604 & 12.1 \\
\hline Gas6 & $5.5181 \mathrm{e}-01$ & 8.79209299 & 13.509 & 0.000237 & 0.011805 & 364.18 & 378.45 & 335.63 & 560.22 & 544.81 & 475.73 \\
\hline G2e3 & $4.7913 \mathrm{e}-01$ & 6.17864935 & 13.503 & 0.000238 & 0.011813 & 58.333 & 61.97 & 61.031 & 86.387 & 85.926 & 80.2 \\
\hline Smoc2 & $5.4161 \mathrm{e}-01$ & 8.17982244 & 13.382 & 0.000254 & 0.012513 & 210.53 & 238.15 & 259.8 & 327.38 & 357.98 & 345. \\
\hline Aspm & $5.4141 \mathrm{e}-01$ & 7.59907070 & 13.359 & 0.000257 & 0.012636 & 171.44 & 140.59 & 161.35 & 213.14 & 220.74 & 255. \\
\hline Etv3 & $5.5829 \mathrm{e}-01$ & 7.65862411 & 13.313 & 0.000264 & 0.012864 & 147.05 & 165.79 & 177.39 & 207.09 & 243.15 & 271. \\
\hline Lrrc7 & $2.0509 \mathrm{e}+00$ & -0.08850242 & 13.29 & 0.000267 & 0.012969 & 0.16738 & 0.66317 & 0.17819 & 1.211 & 1.7306 & 1.13 \\
\hline Dlk2 & $6.1237 \mathrm{e}-01$ & 5.60883789 & 13.289 & 0.000267 & 0.012969 & 31.259 & 42.222 & 42.321 & 58.129 & 67.495 & 50.8 \\
\hline Gm53 & $1.3606 \mathrm{e}+00$ & 1.97764426 & 13.256 & 0.000272 & 0.013073 & 2.4689 & 2.6527 & 1.2473 & 4.9248 & 7.2687 & 4.47 \\
\hline Chn2 & $1.8270 \mathrm{e}+00$ & 1.29086324 & 13.167 & 0.000285 & 0.013589 & 0.62769 & 0.66317 & 1.9601 & 2.4221 & 3.2017 & 5.52 \\
\hline Apol7a & $1.2503 e+00$ & 2.52580974 & 13.162 & 0.000286 & 0.01359 & 3.5987 & 4.4948 & 1.871 & 9.9304 & 6.5764 & 7.45 \\
\hline Fbxo32 & $5.0529 \mathrm{e}-01$ & 6.24969300 & 13.127 & 0.000291 & 0.013816 & 59.839 & 71.033 & 57.556 & 86.548 & 89.301 & 91.6 \\
\hline Pla2g4f & $6.5317 \mathrm{e}-01$ & 7.40256202 & 13.116 & 0.000293 & 0.013867 & 119.01 & 138.6 & 136.85 & 150.01 & 224.03 & 246. \\
\hline N4bp2l1 & 7.8387e-01 & 3.89058408 & 13.042 & 0.000305 & 0.014232 & 10.378 & 9.8739 & 12.295 & 23.009 & 16.441 & 16.3 \\
\hline Sgsm2 & $5.1004 \mathrm{e}-01$ & 5.91081619 & 12.949 & 0.00032 & 0.014817 & 48.708 & 48.632 & 51.319 & 61.278 & 69.312 & 81.0 \\
\hline Gas1 & $8.2573 e-01$ & 6.79358807 & 12.874 & 0.000333 & 0.015296 & 47.788 & 85.917 & 106.56 & 120.05 & 158.96 & 146. \\
\hline Man2b2 & $6.2485 \mathrm{e}-01$ & 4.79196994 & 12.829 & 0.000341 & 0.015593 & 23.015 & 18.642 & 23.432 & 29.468 & 32.623 & 38.3 \\
\hline Reep1 & $1.2450 \mathrm{e}+00$ & 3.95262417 & 12.77 & 0.000352 & 0.015924 & 12.386 & 8.3265 & 6.504 & 33.989 & 20.075 & 10. \\
\hline Ets1 & 5.3267e-01 & 6.48707048 & 12.751 & 0.000356 & 0.016053 & 75.49 & 75.896 & 68.248 & 124.33 & 104.01 & 89.5 \\
\hline 5930430L01Rik & $5.1666 \mathrm{e}-01$ & 5.35461855 & 12.696 & 0.000366 & 0.016423 & 33.1 & 35.001 & 32.609 & 44.081 & 52.525 & 47.5 \\
\hline Tgfbr1 & 5.3971e-01 & 5.41247980 & 12.669 & 0.000372 & 0.016594 & 32.012 & 33.453 & 38.579 & 51.186 & 44.218 & 55.48 \\
\hline Slc43a1 & $1.0753 e+00$ & 2.82577839 & 12.583 & 0.000389 & 0.01726 & 3.9753 & 5.8948 & 3.6529 & 8.3157 & 12.374 & 7.801 \\
\hline Galnt1 & $5.8167 \mathrm{e}-01$ & 6.43380812 & 12.561 & 0.000394 & 0.017431 & 57.454 & 66.538 & 83.84 & 117.87 & 97.608 & 94.8 \\
\hline Zdhhc2 & $9.4806 \mathrm{e}-01$ & 3.52443080 & 12.534 & 0.0004 & 0.017646 & 5.6492 & 6.9264 & 11.048 & 16.47 & 15.576 & 12.9 \\
\hline D6Ertd527e & $1.0794 \mathrm{e}+00$ & 2.37333317 & 12.5 & 0.000407 & 0.017851 & 3.3895 & 3.6843 & 2.6729 & 6.2166 & 8.2205 & 6.31 \\
\hline Serpinb7 & $8.4376 \mathrm{e}-01$ & 5.65002298 & 12.472 & 0.000413 & 0.018089 & 28.748 & 25.937 & 53.19 & 66.929 & 50.881 & 75.212 \\
\hline Soat1 & $1.4559 \mathrm{e}+00$ & 1.72456622 & 12.449 & 0.000418 & 0.018254 & 1.8412 & 1.2527 & 2.0492 & 6.0551 & 5.6246 & 2.454 \\
\hline Tubb6 & $9.6001 \mathrm{e}-01$ & 3.84613126 & 12.447 & 0.000419 & 0.018254 & 9.5827 & 11.2 & 8.2859 & 19.699 & 25.44 & 11.4 \\
\hline Helz2 & $7.5846 \mathrm{e}-01$ & 4.99057385 & 12.418 & 0.000425 & 0.018462 & 23.81 & 20.042 & 26.818 & 31.002 & 33.747 & 54.787 \\
\hline Sirpa & 5.3071e-01 & 6.82386990 & 12.328 & 0.000446 & 0.01929 & 110.01 & 84.223 & 83.216 & 139.51 & 124.26 & 137.71 \\
\hline Hspb2 & $4.7188 \mathrm{e}+00$ & -2.05103255 & 12.276 & 0.000459 & 0.019648 & 0 & 0 & 0 & 0.24221 & 0.34613 & 0.08766 \\
\hline Ncam1 & $2.5434 \mathrm{e}+00$ & -0.5000453 & 12.261 & 0.000463 & 0.019754 & 0.12554 & 0.14737 & 0.26729 & 1.6954 & 1.1249 & 0.26298 \\
\hline Btbd3 & $4.6658 \mathrm{e}-01$ & 6.78033706 & 12.222 & 0.000472 & 0.020086 & 89.466 & 98.149 & 89.007 & 124.33 & 121.23 & 136. \\
\hline Gja1 & $5.5375 \mathrm{e}-01$ & 8.39295298 & 12.175 & 0.000484 & 0.020513 & 233.88 & 264.68 & 318.7 & 370.25 & 415.27 & 413.58 \\
\hline Sestd1 & $7.0322 \mathrm{e}-01$ & 4.20696460 & 12.131 & 0.000496 & 0.020851 & 13.432 & 12.969 & 15.592 & 22.364 & 18.258 & 27.6 \\
\hline B130006D01Rik & $1.0732 \mathrm{e}+00$ & 2.34163418 & 12.111 & 0.000501 & 0.021019 & 2.4689 & 3.6106 & 3.6529 & 6.4588 & 7.0956 & 6.6621 \\
\hline $\mathrm{Abr}$ & $5.1630 \mathrm{e}-01$ & 7.41711391 & 12.096 & 0.000505 & 0.02115 & 134.95 & 125.34 & 161.53 & 191.83 & 195.04 & 216. \\
\hline Bmp2 & $5.5609 \mathrm{e}-01$ & 5.73046345 & 12.085 & 0.000508 & 0.021227 & 35.527 & 41.632 & 51.854 & 58.533 & 62.995 & 67.4 \\
\hline Dbhos & $4.5354 \mathrm{e}+00$ & -2.12684133 & 12.078 & 0.00051 & 0.02123 & 0 & 0 & 0 & 0.16147 & 0.2596 & 0.17532 \\
\hline Gm5126 & $4.5354 \mathrm{e}+00$ & -2.12684133 & 12.078 & 0.00051 & 0.02123 & 0 & 0 & 0 & 0.16147 & 0.2596 & 0.175 \\
\hline
\end{tabular}




\begin{tabular}{|c|c|c|c|c|c|c|c|c|c|c|c|}
\hline Bnc1 & $4.9035 \mathrm{e}-01$ & 6.20498898 & 12.054 & 0.000517 & 0.021453 & 61.597 & 55.927 & 66.288 & 81.865 & 80.388 & \\
\hline Tgfbi & $5.1785 \mathrm{e}-01$ & 9.14732653 & 11.991 & 0.000535 & 0.022106 & 483.32 & 474.98 & 440.4 & 668.89 & 687.5 & 646. \\
\hline Hdac5 & 4.6153e-01 & 7.03472561 & 11.95 & 0.000546 & 0.022555 & 111.52 & 106.7 & 112.44 & 141.53 & 153.51 & 160 \\
\hline Acsbg1 & $5.5861 \mathrm{e}-01$ & 6.90775896 & 11.935 & 0.000551 & 0.022695 & 112.1 & 96.012 & 82.681 & 164.38 & 142.52 & \\
\hline Lmnb1 & $4.7742 \mathrm{e}-01$ & 6.52938345 & 11.885 & 0.000566 & 0.023221 & 70.803 & 77.812 & 82.949 & 100.6 & 119.59 & 101 \\
\hline Dgkq & $4.7219 \mathrm{e}-01$ & 6.33766515 & 11.825 & 0.000585 & 0.023748 & 66.577 & 68.085 & 68.337 & 84.61 & 93.022 & \\
\hline Ccdc88c & $4.7789 \mathrm{e}-01$ & 7.54822878 & 11.818 & 0.000587 & 0.023748 & 158.47 & 145.16 & 165.45 & 206.12 & 227.93 & \\
\hline Ivns1abp & $5.9961 \mathrm{e}-01$ & 9.42547159 & 11.818 & 0.000587 & 0.023748 & 453.94 & 574.01 & 612.27 & 719.03 & 876.31 & 889 \\
\hline Etnk1 & $4.8131 \mathrm{e}-01$ & 5.74889255 & 11.808 & 0.00059 & 0.023818 & 39.963 & 46.422 & 48.201 & 58.129 & 64.639 & 64.6 \\
\hline Kif13a & $5.1903 \mathrm{e}-01$ & 7.89434077 & 11.782 & 0.000598 & 0.02406 & 180.98 & 187.53 & 218.11 & 254.23 & 270.15 & 315. \\
\hline$C 2 c d 4 b$ & $1.4078 \mathrm{e}+00$ & 0.79452277 & 11.737 & 0.000613 & 0.024557 & 0.66953 & 1.179 & 0.89096 & 2.3413 & 2.0768 & 2.71 \\
\hline Hspb1 & $4.7363 \mathrm{e}-01$ & 6.61268606 & 11.623 & 0.000651 & 0.025859 & 83.733 & 74.865 & 86.958 & 127.97 & 106.09 & \\
\hline Gramd1b & $1.0421 \mathrm{e}+00$ & 2.73362720 & 11.616 & 0.000654 & 0.025911 & 4.059 & 5.3054 & 3.4748 & 9.5267 & 10.73 & \\
\hline Mmp1b & $3.4287 \mathrm{e}+00$ & -1.77762523 & 11.595 & 0.000661 & 0.026054 & 0 & 0 & 0.089096 & 0.24221 & 0.43266 & 0.262 \\
\hline Osmr & $5.0057 \mathrm{e}-01$ & 6.37465646 & 11.563 & 0.000673 & 0.026268 & 71.724 & 63.37 & 70.742 & 92.684 & 85.926 & \\
\hline Mturn & $8.3399 \mathrm{e}-01$ & 2.98745260 & 11.542 & 0.00068 & 0.026458 & 5.3981 & 5.379 & 6.1476 & 10.657 & 10.211 & $9.2 C$ \\
\hline Hsf2 & 4.8897e-01 & 5.34917377 & 11.524 & 0.000687 & 0.026621 & 33.979 & 36.253 & 31.184 & 50.379 & 45.862 & 46.1 \\
\hline Scnn1g & $1.3736 \mathrm{e}+00$ & 1.06121084 & 11.506 & 0.000694 & 0.02683 & 1.3391 & 1.2527 & 0.62367 & 3.1487 & 3.2017 & 2.27 \\
\hline Tmem194 & $5.6900 \mathrm{e}-01$ & 4.51339026 & 11.44 & 0.000719 & 0.027538 & 17.91 & 17.979 & 19.067 & 30.276 & 24.835 & \\
\hline Wnt4 & $4.8715 \mathrm{e}-01$ & 8.04563977 & 11.421 & 0.000726 & 0.027722 & 234.34 & 212.44 & 212.94 & 334.32 & 307.53 & \\
\hline Gpr62 & $4.5346 \mathrm{e}+00$ & -2.12737636 & 11.398 & 0.000735 & 0.028024 & 0 & 0 & 0 & 0.24221 & 0.086532 & 0.262 \\
\hline Cidea & $2.2858 \mathrm{e}+00$ & -0.39686976 & 11.339 & 0.000759 & 0.028799 & 0.12554 & 0.22106 & 0.35638 & 1.6954 & 1.2114 & \\
\hline Edar & $1.1248 \mathrm{e}+00$ & 1.88683416 & 11.335 & 0.000761 & 0.028799 & 2.0504 & 2.8737 & 1.871 & 5.3285 & 5.1054 & \\
\hline Gpr27 & $1.5998 \mathrm{e}+00$ & 2.06632706 & 11.334 & 0.000761 & 0.028799 & 0.58584 & 2.9474 & 2.762 & 5.8937 & 7.0091 & \\
\hline Cux1 & $4.8767 \mathrm{e}-01$ & 6.25832156 & 11.299 & 0.000776 & 0.02927 & 64.066 & 66.464 & 60.318 & 77.021 & 101.59 & \\
\hline Rasa3 & $4.4777 \mathrm{e}-01$ & 7.02934027 & 11.293 & 0.000778 & 0.02927 & 112.61 & 109.28 & 109.32 & 140.72 & 151.34 & \\
\hline Ms4a10 & $1.5783 e+00$ & 0.25492787 & 11.284 & 0.000782 & 0.029372 & 0.66953 & 0.22106 & 0.71277 & 1.6954 & 1.3845 & 1.84 \\
\hline Uty & 5.7962e-01 & 6.18943067 & 11.247 & 0.000798 & 0.029906 & 45.235 & 70.075 & 60.229 & 87.759 & 98.3 & \\
\hline Nabp1 & $4.9645 \mathrm{e}-01$ & 5.60260397 & 11.232 & 0.000804 & 0.030071 & 35.234 & 44.875 & 40.717 & 53.608 & 61.005 & 55.4 \\
\hline Mg\|l & $6.4253 \mathrm{e}-01$ & 6.26575129 & 11.23 & 0.000805 & 0.030071 & 65.698 & 69.559 & 44.548 & 87.598 & 116.56 & \\
\hline Pif1 & $5.5726 \mathrm{e}-01$ & 4.51511047 & 11.19 & 0.000822 & 0.030667 & 18.579 & 16.727 & 19.958 & 26.158 & 28.556 & 26.5 \\
\hline 8030423F21Rik & $3.3042 \mathrm{e}+00$ & -1.84387308 & 11.176 & 0.000829 & 0.030853 & 0 & 0.073686 & 0 & 0.32294 & 0.2596 & 0.262 \\
\hline Frmd4b & $4.5392 \mathrm{e}-01$ & 6.60094715 & 11.161 & 0.000835 & 0.031042 & 77.707 & 81.275 & 86.601 & 100.84 & 122.44 & 112 \\
\hline D4Wsu53e & $5.2464 \mathrm{e}-01$ & 7.87031405 & 11.127 & 0.000851 & 0.031566 & 172.45 & 204.18 & 198.95 & 228.8 & 296.03 & 302 \\
\hline Nek2 & $4.9892 \mathrm{e}-01$ & 5.38763086 & 11.063 & 0.000881 & 0.032499 & 30.296 & 35.001 & 38.757 & 49.894 & 51.313 & \\
\hline Zfp804b & $3.3042 \mathrm{e}+00$ & -1.84323067 & 11.051 & 0.000887 & 0.032543 & 0 & 0.073686 & 0 & 0.24221 & 0.34613 & 0.262 \\
\hline Fgf13 & $1.1030 \mathrm{e}+00$ & 2.02066156 & 11.04 & 0.000892 & 0.032678 & 2.3434 & 3.3159 & 1.871 & 5.0056 & 5.6246 & 5.61 \\
\hline Rab11fip4 & $8.2465 \mathrm{e}-01$ & 3.05425975 & 10.988 & 0.000917 & 0.033431 & 5.5655 & 6.2633 & 5.9694 & 12.272 & 9.5185 & 9.64 \\
\hline Lrp4 & $5.5660 \mathrm{e}-01$ & 9.39007626 & 10.97 & 0.000926 & 0.033645 & 532.78 & 501.36 & 594.98 & 716.28 & 747.29 & \\
\hline Bhlhe41 & $1.6564 \mathrm{e}+00$ & -0.14316493 & 10.965 & 0.000928 & 0.033645 & 0.29292 & 0.36843 & 0.53458 & 1.4532 & 1.0384 & 1.13 \\
\hline Mastl & $4.6426 \mathrm{e}-01$ & 5.19491712 & 10.921 & 0.000951 & 0.034231 & 31.259 & 30.211 & 30.56 & 43.92 & 39.805 & 43.3 \\
\hline Syne1 & $5.2868 \mathrm{e}-01$ & 6.68714536 & 10.875 & 0.000975 & 0.034982 & 83.691 & 72.212 & 97.026 & 112.63 & 108.25 & 143 \\
\hline Lamc1 & $4.7764 \mathrm{e}-01$ & 7.27436545 & 10.868 & 0.000979 & 0.035035 & 142.78 & 124.09 & 120.9 & 200.22 & 163.2 & \\
\hline Grik4 & $2.0252 \mathrm{e}+00$ & -0.41232856 & 10.861 & 0.000982 & 0.035035 & 0.41846 & 0.14737 & 0.089096 & 1.4532 & 1.0384 & 0.613 \\
\hline Lamb3 & $5.1210 \mathrm{e}-01$ & 9.57124808 & 10.859 & 0.000983 & 0.035035 & 614.96 & 597.15 & 669.02 & 877.27 & 894.65 & 91 \\
\hline
\end{tabular}




\begin{tabular}{|c|c|c|c|c|c|c|c|c|c|c|c|}
\hline Nlrp5-ps & $1.7306 \mathrm{e}+00$ & -0.12902879 & 10.842 & 0.000992 & 0.035241 & 0.20923 & 0.29474 & 0.71277 & 1.2918 & 1.2114 & 1.2272 \\
\hline Gmip & $5.2893 \mathrm{e}-01$ & 5.08007257 & 10.796 & 0.001017 & 0.03588 & 25.149 & 26.379 & 31.451 & 36.089 & 38.42 & 44.8 \\
\hline Cwh43 & $5.3965 \mathrm{e}-01$ & 5.97161423 & 10.724 & 0.001058 & 0.036821 & 51.47 & 40.159 & 61.655 & 78.555 & 65.678 & 78.3 \\
\hline Ctgf & $2.2523 \mathrm{e}+00$ & -0.55249766 & 10.711 & 0.001065 & 0.036853 & 0.29292 & 0.22106 & 0 & 1.3725 & 1.1249 & 0.350 \\
\hline Сур1а1 & $4.5362 \mathrm{e}+00$ & -2.12695956 & 10.69 & 0.001077 & 0.037009 & 0 & 0 & 0 & 0.16147 & 0.34613 & 0.0876 \\
\hline Ets2 & $4.9365 \mathrm{e}-01$ & 8.57016429 & 10.683 & 0.001081 & 0.037084 & 301.54 & 310.22 & 335.09 & 414.09 & 418.64 & 500. \\
\hline Sorl1 & $4.5036 \mathrm{e}-01$ & 6.56044213 & 10.592 & 0.001136 & 0.03882 & 86.411 & 79.065 & 73.326 & 101.32 & 104.62 & 120.8 \\
\hline Casc5 & $4.3913 \mathrm{e}-01$ & 6.35508769 & 10.587 & 0.001139 & 0.038846 & 71.598 & 66.243 & 70.297 & 91.311 & 88.522 & 102.4 \\
\hline 8430408G22Rik & $1.2256 \mathrm{e}+00$ & 2.26923790 & 10.581 & 0.001143 & 0.038864 & 3.0547 & 3.758 & 1.6037 & 4.4404 & 8.4801 & 7.01 \\
\hline Tgfbr3 & $4.5128 \mathrm{e}-01$ & 6.91351168 & 10.57 & 0.00115 & 0.03904 & 95.994 & 105.37 & 103.98 & 124.82 & 142.43 & 150.0 \\
\hline Zfp518b & 7.7123e-01 & 3.24619159 & 10.536 & 0.001171 & 0.039559 & 7.2812 & 6.1896 & 7.3059 & 11.384 & 10.47 & 13.6 \\
\hline Gramd2 & 5.3927e-01 & 4.40006655 & 10.526 & 0.001177 & 0.039631 & 17.031 & 17.39 & 16.928 & 22.848 & 26.565 & 25.2 \\
\hline Bmp4 & $4.6808 \mathrm{e}-01$ & 5.53872366 & 10.525 & 0.001178 & 0.039631 & 38.121 & 37.948 & 40.717 & 49.087 & 60.832 & \\
\hline Plekha3 & $6.3885 \mathrm{e}-01$ & 4.16979522 & 10.524 & 0.001178 & 0.039631 & 11.173 & 15.179 & 15.859 & 23.09 & 21.114 & 21.0 \\
\hline Dock9 & $4.7820 \mathrm{e}-01$ & 6.89852640 & 10.52 & 0.001181 & 0.039637 & 95.073 & 98.812 & 105.04 & 122.23 & 132.31 & \\
\hline Tbc1d8b & $5.0041 \mathrm{e}-01$ & 4.98980049 & 10.473 & 0.001212 & 0.040225 & 23.434 & 28.222 & 27.174 & 37.057 & 35.651 & 38.4 \\
\hline Sema3c & $5.2692 \mathrm{e}-01$ & 9.04927593 & 10.454 & 0.001224 & 0.040448 & 465.91 & 440.93 & 395.05 & 721.85 & 604.34 & 549.8 \\
\hline Tjap1 & $4.5422 \mathrm{e}-01$ & 5.62200992 & 10.446 & 0.001229 & 0.040562 & 43.017 & 43.106 & 38.133 & 54.012 & 61.611 & 54.8 \\
\hline Zdhhc18 & 4.2040e-01 & 6.23258383 & 10.44 & 0.001233 & 0.04063 & 64.484 & 62.854 & 65.307 & 85.095 & 84.628 & \\
\hline Ccnf & 4.6732e-01 & 5.29007947 & 10.418 & 0.001248 & 0.041022 & 35.36 & 30.58 & 32.164 & 44.404 & 47.679 & \\
\hline Prodh & $5.0823 e-01$ & 5.08856262 & 10.397 & 0.001262 & 0.041378 & 26.405 & 30.285 & 27.353 & 34.312 & 44.131 & 41.0 \\
\hline 9030619P08Rik & $4.3233 e+00$ & -2.2074664 & 10.356 & 0.00129 & 0.041922 & 0 & 0 & 0 & 0.16147 & 0.17306 & 0.175 \\
\hline Tmem100 & $4.3233 e+00$ & -2.2074664 & 10.356 & 0.00129 & 0.041922 & 0 & 0 & 0 & 0.16147 & 0.17306 & 0.175 \\
\hline Gna14 & $5.7212 \mathrm{e}-01$ & 5.66059889 & 10.322 & 0.001314 & 0.042424 & 34.523 & 39.643 & 47.845 & 46.907 & 64.207 & 69.8 \\
\hline Slc9a1 & $4.7750 \mathrm{e}-01$ & 6.35356356 & 10.304 & 0.001327 & 0.042781 & 65.991 & 67.496 & 71.366 & 79.443 & 96.57 & 109. \\
\hline$\| 1 \mathrm{r} 1$ & $4.2164 \mathrm{e}-01$ & 6.20727007 & 10.193 & 0.00141 & 0.045065 & 63.187 & 63.001 & 62.991 & 80.251 & 82.465 & 90.7 \\
\hline Tmc4 & $8.2023 e-01$ & 2.75025694 & 10.166 & 0.00143 & 0.045608 & 4.9378 & 4.9369 & 4.4548 & 8.0735 & 8.9128 & 8.41 \\
\hline Edaradd & $4.2325 \mathrm{e}-01$ & 7.15166385 & 10.159 & 0.001436 & 0.045693 & 120.18 & 126.96 & 116.98 & 166.31 & 168.13 & 153. \\
\hline Slco4a1 & $6.1398 \mathrm{e}-01$ & 3.93101834 & 10.157 & 0.001437 & 0.045693 & 12.721 & 10.832 & 12.295 & 17.358 & 17.912 & 19.7 \\
\hline Tmprss11f & $1.1148 \mathrm{e}+00$ & 2.00352465 & 10.122 & 0.001465 & 0.046283 & 2.2597 & 3.0211 & 2.1383 & 5.9744 & 6.4034 & 3.6817 \\
\hline Kif14 & $5.1911 \mathrm{e}-01$ & 5.02249884 & 10.103 & 0.001481 & 0.046709 & 25.442 & 28.001 & 26.462 & 32.052 & 38.593 & \\
\hline Mt4 & $8.2638 \mathrm{e}-01$ & 4.58266245 & 10.064 & 0.001512 & 0.047483 & 13.14 & 22.916 & 15.681 & 31.729 & 40.151 & 19.6 \\
\hline Nxpe3 & $1.7221 \mathrm{e}+00$ & 1.03934831 & 10.05 & 0.001523 & 0.047669 & 1.0043 & 1.4 & 0.26729 & 2.9872 & 4.6727 & $1.4 \mathrm{C}$ \\
\hline Tbc1d8 & $4.6745 \mathrm{e}-01$ & 6.23013606 & 10.023 & 0.001546 & 0.048123 & 60.634 & 59.243 & 68.96 & 74.761 & 88.176 & 98.0 \\
\hline Zc3h7a & $4.5453 \mathrm{e}-01$ & 7.30416333 & 9.9773 & 0.001585 & 0.048962 & 124.87 & 133.08 & 141.93 & 161.71 & 183.19 & $\angle 0<$. \\
\hline Abca15 & $4.3217 \mathrm{e}+00$ & -2.20824065 & 9.9731 & 0.001588 & 0.049001 & 0 & 0 & 0 & 0.24221 & 0.17306 & 0.087 \\
\hline Mia2 & $4.3219 \mathrm{e}+00$ & -2.20812208 & 9.956 & 0.001603 & 0.04924 & 0 & 0 & 0 & 0.24221 & 0.086532 & 0.17532 \\
\hline Arhgap22 & $7.0602 \mathrm{e}-01$ & 3.43083747 & 9.9262 & 0.001629 & 0.04997 & 7.4485 & 9.3581 & 7.5732 & 12.03 & 14.191 & \\
\hline Prss23 & $4.5787 e-01$ & 6.15867113 & 9.9225 & 0.001633 & 0.049999 & 63.815 & 61.454 & 54.883 & 85.175 & 91.724 & 70.8 \\
\hline
\end{tabular}

LogFC $=$ Log Fold change, $\log C P M=$ Log count per million bases, LR = Log Ratio, FDR = BH corrected P-value, values in columns S7309-S7314 depict TMM normalized relative expression. 
b. downregulated genes in Lgr6 ${ }^{+}$cells

\begin{tabular}{|c|c|c|c|c|c|c|c|c|c|c|c|}
\hline genes & $\log \mathrm{FC}$ & $\log C P M$ & LR & PValue & FDR & S7309 & S7311 & S7313 & S7310 & S7312 & S7314 \\
\hline Scarna6 & -2.4245 & 5.94731743 & 239.01 & $6.46 \mathrm{E}-54$ & $1.35 \mathrm{E}-49$ & 103.94 & 118.12 & 88.651 & 17.842 & 19.47 & 20. \\
\hline Mir6516 & -2.8528 & 6.69509464 & 199.44 & $2.76 \mathrm{E}-45$ & $2.88 \mathrm{E}-41$ & 229.77 & 187.24 & 127.23 & 24.543 & 26.392 & 24.45 \\
\hline Snora74a & -2.7083 & 6.26491762 & 172.5 & $2.1 \mathrm{E}-39$ & $1.46 \mathrm{E}-35$ & 168.22 & 134.48 & 95.689 & 22.121 & 16.96 & 21.91 \\
\hline Snord15a & -3.0501 & 6.98047113 & 149.66 & $2.06 \mathrm{E}-34$ & $1.08 \mathrm{E}-30$ & 333.68 & 189.67 & 150.57 & 29.226 & 26.133 & 26.03 \\
\hline Snord17 & -2.4889 & 7.13452249 & 136.44 & $1.6 \mathrm{E}-31$ & $6.69 \mathrm{E}-28$ & 249.19 & 284.65 & 180.24 & 36.977 & 34.44 & 55.83 \\
\hline Sostdc1 & -1.7485 & 7.32403724 & 114.82 & $8.63 \mathrm{E}-27$ & $3.01 \mathrm{E}-23$ & 281.66 & 232.77 & 225.24 & 86.79 & 65.245 & 68.11 \\
\hline Fst & -2.2292 & 5.17236386 & 110.96 & $6.02 \mathrm{E}-26$ & $1.8 \mathrm{E}-22$ & 65.321 & 59.022 & 52.656 & 16.228 & 13.326 & 8.152 \\
\hline Snord22 & -3.2174 & 6.94020400 & 110.12 & $9.23 E-26$ & $2.41 \mathrm{E}-22$ & 323.89 & 231.52 & 107.63 & 20.265 & 23.969 & 27.08 \\
\hline Snora52 & -2.702 & 5.07102299 & 91.082 & $1.38 \mathrm{E}-21$ & $3.2 \mathrm{E}-18$ & 78.21 & 64.401 & 30.293 & 9.5267 & 8.3071 & 8.76 \\
\hline Hmcn1 & -1.8156 & 8.64596237 & 87.524 & 8.33E-21 & $1.74 \mathrm{E}-17$ & 711.67 & 596.71 & 562.11 & 190.45 & 130.23 & 210.7 \\
\hline Lzts1 & -1.6469 & 4.37415209 & 84.378 & 4.09E-20 & $7.76 \mathrm{E}-17$ & 32.012 & 29.179 & 32.075 & 8.6387 & 10.211 & 10.95 \\
\hline Cxcl12 & -2.4579 & 3.49316866 & 83.055 & $7.98 \mathrm{E}-20$ & $1.39 \mathrm{E}-16$ & 20.295 & 17.021 & 18.532 & 4.1175 & 2.0768 & 3.944 \\
\hline Snord15b & -1.9953 & 8.65610746 & 79.067 & $6.01 \mathrm{E}-19$ & $9.65 \mathrm{E}-16$ & 883.2 & 614.91 & 435.32 & 144.84 & 167.44 & 172.7 \\
\hline Aadacl3 & -1.4765 & 5.51788602 & 77.642 & $1.23 \mathrm{E}-18$ & $1.84 \mathrm{E}-15$ & 69.966 & 68.38 & 62.813 & 19.053 & 24.056 & 29.27 \\
\hline Scarna10 & -2.0741 & 8.32236244 & 77.048 & $1.67 \mathrm{E}-18$ & $2.32 \mathrm{E}-15$ & 454.44 & 663.1 & 433.36 & 161.39 & 124.09 & 82.75 \\
\hline Rprl3 & -1.9896 & 7.63725499 & 71.701 & $2.5 \mathrm{E}-17$ & $3.08 \mathrm{E}-14$ & 312.34 & 374.91 & 265.77 & 71.935 & 113.88 & 54.17 \\
\hline Trpc4 & -2.2411 & 3.89881518 & 69.096 & $9.38 \mathrm{E}-17$ & $1.09 \mathrm{E}-13$ & 29.543 & 25.2 & 17.641 & 6.9432 & 4.1535 & 4.207 \\
\hline Snora5c & -2.9615 & 4.00837572 & 67.249 & $2.39 \mathrm{E}-16$ & $2.5 \mathrm{E}-13$ & 41.009 & 29.843 & 12.652 & 4.279 & 3.2017 & 3.243 \\
\hline Ptprv & -1.4416 & 5.35214684 & 66.088 & $4.31 \mathrm{E}-16$ & 4.29E-13 & 62.392 & 64.917 & 50.785 & 26.966 & 18.604 & 19.98 \\
\hline Aldh3a1 & -1.5708 & 6.15322889 & 64.904 & $7.86 \mathrm{E}-16$ & $7.46 \mathrm{E}-13$ & 116.92 & 85.107 & 116.45 & 46.665 & 27.69 & 32.78 \\
\hline Inhbb & -2.4236 & 4.39248178 & 61.954 & $3.52 \mathrm{E}-15$ & $2.82 \mathrm{E}-12$ & 40.381 & 39.569 & 24.769 & 10.253 & 5.6246 & 3.594 \\
\hline Runx1 & -1.7567 & 4.89986042 & 60.82 & $6.25 \mathrm{E}-15$ & $4.66 \mathrm{E}-12$ & 52.098 & 47.454 & 37.42 & 16.47 & 8.7397 & 15.3 \\
\hline Lphn3 & -1.9446 & 5.96124373 & 60.665 & $6.77 \mathrm{E}-15$ & 4.87E-12 & 125.08 & 100.95 & 69.228 & 36.573 & 21.72 & 18.40 \\
\hline 5730408K05Rik & -1.4932 & 5.84555060 & 60.201 & 8.56E-15 & $5.96 \mathrm{E}-12$ & 85.365 & 103.82 & 64.327 & 34.716 & 32.19 & 23.14 \\
\hline Fam132a & -1.7062 & 6.67563546 & 59.371 & $1.31 \mathrm{E}-14$ & $8.52 \mathrm{E}-12$ & 164.66 & 153.27 & 150.48 & 66.768 & 46.9 & 29.8 \\
\hline Rprl2 & -2.5996 & 2.93948474 & 58.763 & $1.78 \mathrm{E}-14$ & 1.13E-11 & 11.8 & 16.727 & 9.7115 & 1.7762 & 3.0286 & 1.49 \\
\hline Ephx2 & -2.3102 & 3.78903813 & 58.162 & $2.41 \mathrm{E}-14$ & $1.48 \mathrm{E}-11$ & 27.702 & 22.548 & 17.285 & 6.2973 & 4.9323 & 2.366 \\
\hline Snora68 & -2.7941 & 3.21601078 & 57.121 & 4.1E-14 & $2.45 \mathrm{E}-11$ & 19.04 & 20.411 & 7.5732 & 2.3413 & 2.3364 & 2.10 \\
\hline Mir1949 & -2.7708 & 3.28104116 & 56.087 & $6.93 \mathrm{E}-14$ & $4.02 \mathrm{E}-11$ & 23.141 & 17.832 & 8.0187 & 2.745 & 2.2498 & 2.191 \\
\hline Snora17 & -2.1567 & 3.70751232 & 55.668 & $8.58 \mathrm{E}-14$ & $4.84 \mathrm{E}-11$ & 25.944 & 23.727 & 12.83 & 5.3285 & 3.8074 & 4.908 \\
\hline Rmrp & -1.8943 & 13.38180123 & 55.521 & $9.25 \mathrm{E}-14$ & $5.08 \mathrm{E}-11$ & 14861 & 19178 & 16428 & 5477.7 & 4995.4 & 3102 \\
\hline Snora70 & -2.1428 & 3.99932939 & 54.941 & $1.24 \mathrm{E}-13$ & $6.65 \mathrm{E}-11$ & 32.305 & 28.958 & 15.414 & 5.8937 & 4.3266 & 7.188 \\
\hline Esyt3 & -1.3876 & 4.33164005 & 54.809 & $1.33 \mathrm{E}-13$ & $6.83 \mathrm{E}-11$ & 31.677 & 27.779 & 26.907 & 11.868 & 9.0859 & 12.09 \\
\hline $\mathrm{Fhl} 2$ & -1.4977 & 4.45915414 & 54.79 & $1.34 \mathrm{E}-13$ & $6.83 \mathrm{E}-11$ & 38.205 & 28.516 & 29.58 & 13.321 & 9.1724 & 11.65 \\
\hline Snora61 & -1.9231 & 4.47974657 & 52.168 & $5.1 \mathrm{E}-13$ & $2.53 \mathrm{E}-10$ & 35.778 & 48.19 & 20.67 & 8.5579 & 10.384 & 8.678 \\
\hline Prkcq & -1.9167 & 3.59063324 & 52.027 & $5.47 \mathrm{E}-13$ & $2.66 \mathrm{E}-10$ & 20.211 & 21.074 & 14.612 & 4.1175 & 4.067 & 6.662 \\
\hline Barx2 & -1.0292 & 7.55479132 & 51.971 & $5.63 \mathrm{E}-13$ & 2.67E-10 & 259.9 & 233.07 & 263.37 & 120.46 & 116.04 & 134.1 \\
\hline Fam117a & -1.3568 & 4.18477027 & 51.309 & $7.89 \mathrm{E}-13$ & $3.66 \mathrm{E}-10$ & 27.158 & 25.422 & 24.947 & 11.707 & 9.5185 & 9.02 \\
\hline Snora78 & -2.4094 & 4.19056664 & 51.255 & $8.11 \mathrm{E}-13$ & $3.68 \mathrm{E}-10$ & 37.117 & 36.253 & 17.196 & 5.167 & 8.5667 & 3.331 \\
\hline $\mathrm{Nt} 5 \mathrm{dc} 2$ & -1.2541 & 4.97463750 & 51.162 & $8.51 \mathrm{E}-13$ & $3.78 \mathrm{E}-10$ & 49.503 & 46.938 & 35.371 & 17.681 & 17.826 & 19.85 \\
\hline Wnt11 & -1.6922 & 3.75013343 & 51.045 & $9.03 \mathrm{E}-13$ & 3.93E-10 & 22.639 & 21.443 & 16.394 & 7.7506 & 5.7976 & 5.171 \\
\hline Add2 & -2.5762 & 3.96126203 & 50.374 & $1.27 \mathrm{E}-12$ & $5.42 \mathrm{E}-10$ & 38.414 & 26.085 & 13.632 & 6.2973 & 2.769 & 4.032 \\
\hline Dlc1 & -1.2486 & 5.55254151 & 49.686 & $1.8 \mathrm{E}-12$ & $7.53 E-10$ & 74.778 & 63.443 & 58.893 & 27.773 & 21.46 & 33.83 \\
\hline
\end{tabular}




\begin{tabular}{|c|c|c|c|c|c|c|c|c|c|c|c|}
\hline II11ra1 & -1.0462 & 5.87628748 & 49.494 & 1.99E-12 & 7.99E-10 & 79.256 & 86.802 & 70.564 & 43.274 & 38.507 & $32.78 !$ \\
\hline Igsf9 & -1.0779 & 6.91060853 & 48.815 & $2.81 \mathrm{E}-12$ & $1.11 \mathrm{E}-09$ & 158.13 & 177.95 & 152.89 & 67.737 & 72.254 & 91.69 \\
\hline Nbea & -2.3158 & 4.40560132 & 48.399 & $3.48 \mathrm{E}-12$ & $1.32 \mathrm{E}-09$ & 47.872 & 39.938 & 16.305 & 8.235 & 7.7879 & 4.908 \\
\hline Krt73 & -3.791 & 2.91818904 & 48.247 & 3.76E-12 & $1.4 \mathrm{E}-09$ & 20.839 & 14.737 & 4.4548 & 0.96882 & 1.5576 & 0.3506 \\
\hline Gstm5 & -1.3049 & 4.44686575 & 47.889 & $4.51 \mathrm{E}-12$ & $1.62 \mathrm{E}-09$ & 33.686 & 30.137 & 28.333 & 14.936 & 11.682 & 10.69 \\
\hline Smco4 & -1.2374 & 5.08125663 & 46.714 & $8.21 \mathrm{E}-12$ & $2.91 \mathrm{E}-09$ & 43.268 & 53.496 & 45.083 & 21.718 & 23.104 & 15.25: \\
\hline Slco2b1 & -2.5196 & 4.18946193 & 45.82 & $1.3 \mathrm{E}-11$ & 4.44E-09 & 36.95 & 29.179 & 25.571 & 8.3964 & 5.8842 & 1.665 \\
\hline Snora21 & -2.9291 & 3.77528598 & 45.665 & $1.4 \mathrm{E}-11$ & $4.73 \mathrm{E}-09$ & 36.113 & 25.643 & 8.7314 & 3.0679 & 1.9037 & 4.295: \\
\hline Susd2 & -1.3282 & 7.84147011 & 45.325 & $1.67 \mathrm{E}-11$ & 5.53E-09 & 410.46 & 335.79 & 236.64 & 126.03 & 114.22 & 151.3 \\
\hline Adh7 & -1.0641 & 8.08113425 & 45.015 & $1.96 \mathrm{E}-11$ & $6.28 \mathrm{E}-09$ & 365.61 & 370.2 & 362.53 & 158.4 & 210.88 & 156.0 \\
\hline Dsc1 & -1.2284 & 5.76672804 & 44.299 & $2.82 \mathrm{E}-11$ & $8.92 \mathrm{E}-09$ & 92.563 & 77.001 & 58.18 & 31.567 & 27.604 & 38. \\
\hline Snora43 & -2.3416 & 3.47657071 & 44.068 & $3.17 \mathrm{E}-11$ & $9.86 \mathrm{E}-09$ & 28.455 & 15.474 & 9.9788 & 3.7138 & 3.8074 & 3.155 \\
\hline Scarna3a & -2.6009 & 3.33653567 & 44.045 & $3.21 \mathrm{E}-11$ & $9.86 \mathrm{E}-09$ & 24.982 & 17.832 & 7.3059 & 3.3101 & 2.6825 & 2.279 \\
\hline Nedd9 & -1.1864 & 6.09400967 & 43.902 & $3.45 \mathrm{E}-11$ & $1.05 \mathrm{E}-08$ & 105.87 & 86.949 & 90.967 & 54.496 & 35.651 & $34.53 i$ \\
\hline Sulf2 & -2.0249 & 5.05338805 & 43.306 & $4.68 \mathrm{E}-11$ & $1.4 \mathrm{E}-08$ & 73.983 & 55.854 & 28.422 & 17.681 & 12.374 & 8.853 \\
\hline Sox9 & -1.7671 & 4.38901675 & 43.219 & $4.89 \mathrm{E}-11$ & $1.44 \mathrm{E}-08$ & 37.745 & 33.38 & 24.769 & 13.886 & 8.3936 & 5.873 \\
\hline Aqp3 & -1.0421 & 8.16438793 & 42.434 & $7.31 \mathrm{E}-11$ & $2.12 \mathrm{E}-08$ & 398.58 & 410.8 & 348.46 & 210.4 & 194.78 & 157.0 \\
\hline Klk8 & -1.3421 & 6.90682417 & 42.233 & $8.1 \mathrm{E}-11$ & $2.32 \mathrm{E}-08$ & 228.94 & 160.41 & 125.63 & 78.555 & 69.139 & 55.57 \\
\hline Smpdl3a & -1.5715 & 5.57138943 & 40.538 & $1.93 \mathrm{E}-10$ & 5.37E-08 & 90.638 & 63.517 & 58.002 & 24.059 & 14.71 & 32.69 \\
\hline Chil1 & -2.3551 & 2.97300496 & 39.789 & $2.83 \mathrm{E}-10$ & 7.67E-08 & 18.412 & 10.684 & 8.6423 & 3.7138 & 1.8172 & 1.840 \\
\hline Snora64 & -2.0082 & 2.99097489 & 39.602 & $3.11 \mathrm{E}-10$ & $8.23 \mathrm{E}-08$ & 14.52 & 14.737 & 7.5732 & 2.745 & 3.1152 & 3.331 \\
\hline Clic4 & -1.0188 & 7.58207682 & 39.576 & $3.16 \mathrm{E}-10$ & $8.24 \mathrm{E}-08$ & 294.09 & 232.48 & 242.25 & 147.34 & 110.07 & 122.1 \\
\hline Dpysl3 & -2.2066 & 2.94324189 & 39.311 & $3.61 \mathrm{E}-10$ & $9.32 \mathrm{E}-08$ & 12.177 & 14.737 & 9.8006 & 1.9376 & 1.6441 & 4.38 \\
\hline Zfp365 & -1.5454 & 3.57578686 & 38.82 & $4.65 \mathrm{E}-10$ & $1.18 \mathrm{E}-07$ & 20.337 & 17.537 & 14.255 & 7.2662 & 5.8842 & 4.733 \\
\hline Hoxc13 & -2.4109 & 1.62925467 & 38.073 & $6.81 \mathrm{E}-10$ & $1.71 \mathrm{E}-07$ & 5.3144 & 4.4948 & 4.633 & 1.2918 & 0.60572 & 0.7889 \\
\hline $\mathrm{Ccl} 2$ & -3.5745 & 0.22221791 & 36.644 & $1.42 \mathrm{E}-09$ & $3.48 \mathrm{E}-07$ & 1.8412 & 1.5474 & 1.9601 & 0.16147 & 0 & 0.2629 \\
\hline Vdr & -1.101 & 7.15708151 & 36.535 & $1.5 \mathrm{E}-09$ & $3.6 \mathrm{E}-07$ & 246.81 & 188.86 & 147.19 & 94.864 & 80.561 & 96.51 \\
\hline Emb & -1.8464 & 5.24292604 & 36.196 & $1.78 \mathrm{E}-09$ & 4.19E-07 & 71.933 & 59.759 & 44.726 & 26.077 & 14.624 & 8.327 \\
\hline Rpph1 & -1.6983 & 12.81501017 & 35.724 & $2.27 \mathrm{E}-09$ & $5.25 \mathrm{E}-07$ & 10748 & 12733 & 9570.3 & 4275 & 3986.6 & 1923. \\
\hline Ephb2 & -1.8157 & 4.08260886 & 35.689 & 2.31E-09 & $5.25 \mathrm{E}-07$ & 33.644 & 21.369 & 22.809 & 9.2845 & 3.6343 & 9.204 \\
\hline Lgals9 & -1.2841 & 4.38389914 & 35.4 & $2.69 \mathrm{E}-09$ & $6.03 \mathrm{E}-07$ & 36.113 & 28.516 & 22.987 & 11.787 & 9.7781 & 14.5 \\
\hline Snora15 & -2.7232 & 2.31291426 & 35.223 & $2.94 \mathrm{E}-09$ & $6.53 \mathrm{E}-07$ & 9.4571 & 10.463 & 4.4548 & 0.56515 & 1.1249 & 2.016 \\
\hline Angpt|2 & -1.2612 & 5.73545213 & 34.988 & $3.32 \mathrm{E}-09$ & $7.29 \mathrm{E}-07$ & 90.596 & 65.285 & 68.604 & 43.678 & 24.921 & 25.07 \\
\hline Egr2 & -1.7902 & 5.91322282 & 34.578 & 4.1E-09 & $8.91 \mathrm{E}-07$ & 99.969 & 106.7 & 72.613 & 41.256 & 27.171 & 12.27 \\
\hline Hpgd & -1.6964 & 2.94487716 & 34.346 & 4.61E-09 & $9.93 \mathrm{E}-07$ & 13.307 & 11.642 & 9.1769 & 4.3597 & 3.4613 & $2.717 !$ \\
\hline Pappa & -1.9332 & 2.59023372 & 34.059 & $5.35 \mathrm{E}-09$ & $1.14 \mathrm{E}-06$ & 11.173 & 7.5896 & 8.6423 & 2.9872 & 1.5576 & 2.62 \\
\hline Fam83a & -1.2124 & 4.11231589 & 33.736 & $6.31 \mathrm{E}-09$ & $1.33 \mathrm{E}-06$ & 26.07 & 23.432 & 22.007 & 8.8809 & 9.0859 & 12.97 \\
\hline Pdzk1ip1 & -1.0422 & 5.31979118 & 33.686 & $6.48 \mathrm{E}-09$ & $1.34 \mathrm{E}-06$ & 58.542 & 49.369 & 52.478 & 22.525 & 22.066 & 33.39 \\
\hline Smpdl3b & -1.6452 & 3.43731212 & 33.367 & 7.63E-09 & $1.56 \mathrm{E}-06$ & 18.287 & 11.569 & 18.354 & 5.9744 & 3.4613 & 5.960 \\
\hline Adamtsl1 & -1.271 & 5.02426198 & 33.254 & 8.09E-09 & $1.64 \mathrm{E}-06$ & 44.273 & 50.99 & 41.875 & 12.756 & 17.826 & $26.29 !$ \\
\hline Snora44 & -1.9663 & 3.52852042 & 32.911 & $9.65 \mathrm{E}-09$ & $1.94 \mathrm{E}-06$ & 25.861 & 18.2 & 9.4442 & 5.9744 & 3.9805 & 3.769 \\
\hline Lrrn1 & -2.3854 & 1.57285668 & 32.84 & $1 \mathrm{E}-08$ & $1.99 \mathrm{E}-06$ & 4.2264 & 6.2633 & 3.3857 & 0.72662 & 0.95185 & 0.9642 \\
\hline Nudt4 & -0.74315 & 6.17872150 & 32.219 & $1.38 \mathrm{E}-08$ & $2.66 \mathrm{E}-06$ & 88.043 & 93.507 & 89.898 & 55.949 & 53.996 & 52.15 \\
\hline Vsnl1 & -1.6442 & 2.80055103 & 31.671 & $1.83 \mathrm{E}-08$ & $3.47 \mathrm{E}-06$ & 11.675 & 9.2844 & 9.6224 & 4.1982 & 2.5094 & 3.068 \\
\hline Cldn4 & -1.4779 & 4.06294714 & 30.469 & 3.39E-08 & $6.11 \mathrm{E}-06$ & 29.166 & 23.948 & 19.512 & 12.837 & 6.836 & 6.35 \\
\hline
\end{tabular}




\begin{tabular}{|c|c|c|c|c|c|c|c|c|c|c|c|}
\hline Orm1 & -1.2777 & 3.65157140 & 30.433 & $3.46 \mathrm{E}-08$ & $6.17 \mathrm{E}-06$ & 20.63 & 15.327 & 16.394 & 8.3964 & 6.7495 & 6.486 \\
\hline Pdlim4 & -2.0213 & 2.94977876 & 30.192 & $3.91 \mathrm{E}-08$ & $6.93 \mathrm{E}-06$ & 11.256 & 12.453 & 12.473 & 4.7634 & 2.9421 & 1.139 \\
\hline Otud7a & -2.243 & 3.34791627 & 30.137 & 4.03E-08 & 7.07E-06 & 8.8713 & 14.811 & 26.194 & 3.3101 & 1.9037 & 5.259 \\
\hline Ldhb & -0.98146 & 4.41409690 & 30.074 & 4.16E-08 & $7.24 \mathrm{E}-06$ & 28.832 & 27.853 & 27.442 & 16.793 & 12.461 & 13.3 \\
\hline Gsdma3 & -2.0685 & 2.49508418 & 29.924 & 4.49E-08 & 7.76E-06 & 9.4571 & 5.6738 & 11.137 & 2.8257 & 2.1633 & 1.227: \\
\hline Dach1 & -0.98894 & 4.73738870 & 28.908 & $7.59 \mathrm{E}-08$ & $1.28 \mathrm{E}-05$ & 32.765 & 34.411 & 38.579 & 15.905 & 15.403 & $21.91 !$ \\
\hline Itpkb & -0.98804 & 5.68808079 & 28.88 & 7.7E-08 & $1.29 \mathrm{E}-05$ & 78.084 & 78.401 & 48.112 & 35.846 & 35.392 & 32.08 \\
\hline Bdh1 & -0.88527 & 4.86305673 & 28.654 & $8.66 \mathrm{E}-08$ & $1.43 \mathrm{E}-05$ & 42.85 & 35.369 & 34.124 & 20.91 & 19.643 & 20.42 \\
\hline Stard5 & -0.69431 & 6.56027038 & 28.598 & $8.91 \mathrm{E}-08$ & $1.47 \mathrm{E}-05$ & 121.19 & 117.09 & 110.92 & 74.518 & 69.399 & 71.96 \\
\hline Faah & -1.0099 & 6.12958356 & 28.496 & $9.39 \mathrm{E}-08$ & $1.53 \mathrm{E}-05$ & 91.014 & 89.675 & 99.253 & 38.43 & 36.949 & 63.64 \\
\hline Klhl29 & -1.5304 & 2.68287255 & 28.046 & $1.18 \mathrm{E}-07$ & $1.9 \mathrm{E}-05$ & 8.7039 & 8.4002 & 10.692 & 3.5523 & 2.596 & 3.41 \\
\hline Mal2 & -1.2815 & 4.30591540 & 27.94 & $1.25 \mathrm{E}-07$ & $1.98 \mathrm{E}-05$ & 36.406 & 26.822 & 19.601 & 11.626 & 8.9128 & 13.67! \\
\hline Mycl & -0.73761 & 7.32151333 & 27.85 & $1.31 \mathrm{E}-07$ & $2.06 \mathrm{E}-05$ & 212.74 & 207.79 & 178.55 & 119.89 & 123.05 & 116. \\
\hline Flrt3 & -0.76579 & 7.00010878 & 27.595 & $1.5 \mathrm{E}-07$ & $2.3 \mathrm{E}-05$ & 172.24 & 157.76 & 152.8 & 93.088 & 82.551 & 108.4 \\
\hline Shroom3 & -1.2931 & 5.06434466 & 27.484 & $1.58 \mathrm{E}-07$ & $2.42 \mathrm{E}-05$ & 48.499 & 50.696 & 42.41 & 13.079 & 15.23 & 29.54 \\
\hline $\mathrm{Ccl} 7$ & -4.768 & -0.52674233 & 26.91 & $2.13 \mathrm{E}-07$ & $3.18 \mathrm{E}-05$ & 0.87876 & 0.58948 & 1.6037 & 0.080735 & 0 & \\
\hline Ndrg2 & -1.1024 & 5.45186435 & 26.628 & $2.47 \mathrm{E}-07$ & $3.63 \mathrm{E}-05$ & 72.184 & 66.464 & 39.381 & 32.133 & 28.209 & 22.70 \\
\hline Gprin3 & -2.0443 & 2.94381743 & 26.607 & $2.49 \mathrm{E}-07$ & $3.64 \mathrm{E}-05$ & 17.492 & 11.642 & 6.4149 & 4.3597 & 2.2498 & 2.01 \\
\hline Fam84a & -0.78841 & 7.51031003 & 26.514 & $2.62 \mathrm{E}-07$ & $3.8 \mathrm{E}-05$ & 252.5 & 236.83 & 202.6 & 151.78 & 129.02 & 119.9: \\
\hline Acot1 & -1.3445 & 4.87443394 & 26.402 & $2.77 \mathrm{E}-07$ & $3.99 \mathrm{E}-05$ & 41.678 & 49.517 & 34.124 & 23.817 & 15.835 & 9.642 \\
\hline Rnf152 & -0.82811 & 5.16702367 & 26.35 & $2.85 \mathrm{E}-07$ & 4.05E-05 & 44.022 & 45.096 & 48.112 & 22.444 & 23.883 & 30.5 \\
\hline Tbc1d30 & -2.3591 & 0.67551284 & 26.111 & $3.22 \mathrm{E}-07$ & $4.52 \mathrm{E}-05$ & 1.9667 & 2.5053 & 2.6729 & 0.56515 & 0.43266 & 0.350 \\
\hline Impdh1 & -1.2243 & 4.07046700 & 25.662 & 4.07E-07 & $5.63 \mathrm{E}-05$ & 23.978 & 20.632 & 25.125 & 8.4772 & 7.2687 & 14.1 \\
\hline Snord23 & -2.2193 & 1.24756747 & 25.488 & $4.45 \mathrm{E}-07$ & $6.12 \mathrm{E}-05$ & 2.5107 & 4.5685 & 3.8311 & 0.56515 & 0.95185 & $0.78 \varepsilon$ \\
\hline Scarna3b & -2.565 & 1.01417275 & 25.256 & $5.02 \mathrm{E}-07$ & $6.81 \mathrm{E}-05$ & 3.7661 & 3.758 & 1.5146 & 0.56515 & 0.60572 & 0.3506 \\
\hline Itpripl1 & -1.0998 & 4.40061270 & 25.256 & $5.02 \mathrm{E}-07$ & $6.81 \mathrm{E}-05$ & 34.606 & 28.369 & 22.363 & 15.986 & 13.932 & 9.99 \\
\hline Grin1 & -2.2323 & 1.17328469 & 25.027 & $5.65 \mathrm{E}-07$ & $7.56 \mathrm{E}-05$ & 3.3058 & 3.5369 & 3.2966 & 0.24221 & 1.1249 & $0.78 \varepsilon$ \\
\hline Psrc1 & -0.81142 & 5.17009824 & 25.016 & $5.68 \mathrm{E}-07$ & $7.56 \mathrm{E}-05$ & 51.805 & 42.517 & 42.41 & 29.791 & 25.267 & 22.9 \\
\hline Prickle2 & -0.8513 & 5.94839724 & 25.003 & $5.72 \mathrm{E}-07$ & 7.57E-05 & 90.763 & 86.359 & 60.229 & 38.511 & 44.131 & 49.17 \\
\hline Lurap1l & -1.9709 & 2.65382145 & 24.95 & $5.88 \mathrm{E}-07$ & $7.73 \mathrm{E}-05$ & 12.554 & 11.274 & 4.8112 & 3.3101 & 2.2498 & 1.75 \\
\hline Pxdc1 & -0.78122 & 5.59768935 & 24.806 & $6.34 \mathrm{E}-07$ & $8.23 \mathrm{E}-05$ & 60.969 & 52.685 & 69.406 & 37.138 & 30.892 & 38.3 \\
\hline Rps6ka6 & -0.86877 & 4.75968575 & 24.626 & $6.96 \mathrm{E}-07$ & $8.97 \mathrm{E}-05$ & 38.038 & 36.695 & 29.402 & 20.91 & 19.21 & 17.00 \\
\hline Kif26b & -1.7031 & 4.76038273 & 24.537 & $7.29 \mathrm{E}-07$ & $9.34 \mathrm{E}-05$ & 43.603 & 33.969 & 45.795 & 8.8809 & 6.3168 & 22.70 \\
\hline Epcam & -0.88394 & 6.76887622 & 24.35 & $8.03 \mathrm{E}-07$ & 0.000102 & 156.08 & 117.9 & 149.59 & 97.609 & 63.255 & 8.6 \\
\hline $\mathrm{Scd} 2$ & -1.1942 & 5.20857298 & 24.136 & $8.98 \mathrm{E}-07$ & 0.000112 & 60.425 & 59.759 & 33.055 & 19.296 & 17.739 & 30.06 \\
\hline Nqo1 & -1.1127 & 4.47043557 & 23.927 & $1 \mathrm{E}-06$ & 0.000124 & 35.694 & 29.99 & 24.234 & 18.811 & 11.509 & 1.3 \\
\hline Gfra1 & -2.2732 & 3.17253588 & 23.817 & $1.06 \mathrm{E}-06$ & 0.000131 & 23.643 & 12.969 & 6.3258 & 4.1982 & 3.4613 & 1.227 \\
\hline 2010107E04Rik & -0.75958 & 5.85959543 & 23.608 & $1.18 \mathrm{E}-06$ & 0.000145 & 75.113 & 77.222 & 65.931 & 49.41 & 45.602 & 33.9 \\
\hline Apobec1 & -1.2694 & 3.86634032 & 23.478 & $1.26 \mathrm{E}-06$ & 0.000154 & 25.568 & 19.895 & 15.235 & 11.303 & 7.3552 & 6.57 \\
\hline Chchd10 & -1.4439 & 2.74392297 & 23.306 & $1.38 \mathrm{E}-06$ & 0.000167 & 10.252 & 8.916 & 9.266 & 4.7634 & 3.1152 & 2.54 \\
\hline Flrt2 & -1.1553 & 5.47385956 & 23.174 & $1.48 \mathrm{E}-06$ & 0.000177 & 75.741 & 52.317 & 54.883 & 24.543 & 18.172 & 39.5 \\
\hline Mmp2 & -0.92001 & 4.41281705 & 22.797 & $1.8 \mathrm{E}-06$ & 0.00021 & 27.493 & 33.011 & 22.274 & 13.16 & 15.316 & 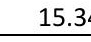 \\
\hline Fam167a & -0.80543 & 5.15202363 & 22.563 & 2.03E-06 & 0.000232 & 49.713 & 39.201 & 45.974 & 27.208 & 20.941 & 29.1 \\
\hline Sort1 & -0.96141 & 6.41633994 & 22.513 & $2.09 \mathrm{E}-06$ & 0.000236 & 141.44 & 119.81 & 76.177 & 56.272 & 50.102 & 67.14 \\
\hline Lrat & -1.9991 & 4.38469135 & 22.42 & 2.19E-06 & 0.000245 & 42.264 & 42.517 & 13.899 & 13.886 & 6.836 & 3.9 \\
\hline
\end{tabular}




\begin{tabular}{|c|c|c|c|c|c|c|c|c|c|c|c|}
\hline Aox4 & -2.1787 & 2.87609483 & 22.38 & $2.24 \mathrm{E}-06$ & 0.000248 & 14.269 & 16.874 & 3.3857 & 2.5835 & 2.4229 & 2.629 \\
\hline Ifitm2 & -0.79582 & 5.84570837 & 22.301 & $2.33 \mathrm{E}-06$ & 0.000258 & 76.787 & 71.107 & 70.297 & 51.105 & 43.612 & 30.94 \\
\hline Cplx2 & -1.7254 & 1.65803546 & 22.273 & $2.36 \mathrm{E}-06$ & 0.00026 & 4.6867 & 4.9369 & 3.9202 & 0.96882 & 1.3845 & 1.753 \\
\hline Snora16a & -1.619 & 4.06082922 & 22.084 & $2.61 \mathrm{E}-06$ & 0.000279 & 35.234 & 28.443 & 10.335 & 8.4772 & 6.663 & \\
\hline Tnc & -0.88522 & 6.08703999 & 21.841 & $2.96 \mathrm{E}-06$ & 0.000314 & 109.38 & 72.801 & 81.434 & 56.03 & 35.997 & 50.84 \\
\hline Endod1 & -1.173 & 5.74967778 & 21.564 & $3.42 \mathrm{E}-06$ & 0.000361 & 100.26 & 71.18 & 50.963 & 46.1 & 24.315 & 28 \\
\hline 4933431E20Rik & -1.163 & 3.45806326 & 21.557 & $3.44 \mathrm{E}-06$ & 0.000361 & 18.036 & 14.369 & 12.117 & 6.2973 & 5.7111 & 7.97 \\
\hline Sox7 & -0.95499 & 6.01039497 & 21.038 & $4.5 \mathrm{E}-06$ & 0.000466 & 88.336 & 72.58 & 93.462 & 35.443 & 33.747 & 62.06 \\
\hline Eya2 & -1.7817 & 3.91824714 & 20.615 & $5.62 \mathrm{E}-06$ & 0.000572 & 30.38 & 23.948 & 14.523 & 10.98 & 6.4899 & 2.542 \\
\hline Apcdd1 & -0.71178 & 7.07018131 & 20.4 & $6.28 \mathrm{E}-06$ & 0.000628 & 189.98 & 158.65 & 151.11 & 106.89 & 84.369 & 114.0 \\
\hline Pls3 & -0.63164 & 7.37207534 & 20.377 & $6.36 \mathrm{E}-06$ & 0.000633 & 212.83 & 199.02 & 191.47 & 138.14 & 118.12 & 133 \\
\hline Runx2 & -1.6671 & 2.63720401 & 20.322 & $6.54 \mathrm{E}-06$ & 0.000648 & 12.261 & 8.3265 & 6.504 & 4.3597 & 2.2498 & 1.92 \\
\hline Snord8 & -1.6647 & 1.98557796 & 20.286 & $6.67 \mathrm{E}-06$ & 0.000657 & 6.8209 & 6.337 & 3.742 & 1.534 & 1.9902 & 1.8 \\
\hline Slc25a13 & -0.59712 & 6.44382223 & 20.277 & $6.7 \mathrm{E}-06$ & 0.000657 & 105.12 & 106.4 & 102.28 & 72.581 & 64.12 & 70.74 \\
\hline Ifngr2 & -0.72051 & 5.45520399 & 20.254 & $6.78 \mathrm{E}-06$ & 0.000662 & 61.304 & 51.506 & 50.161 & 38.914 & 29.334 & 30. \\
\hline Aqp9 & -1.4799 & 2.80623143 & 20.164 & 7.11E-06 & 0.00069 & 8.7039 & 7.5896 & 13.899 & 2.5835 & 3.5478 & \\
\hline Gsdmc & -1.1318 & 4.04597005 & 20.068 & 7.47E-06 & 0.000712 & 26.154 & 19.453 & 21.472 & 9.1231 & 7.0091 & 14.5 \\
\hline Gab2 & -1.8409 & 1.31442166 & 20.059 & 7.51E-06 & 0.000712 & 3.515 & 3.9053 & 3.2966 & 0.96882 & 0.51919 & 1.4 \\
\hline Fzd7 & -0.80048 & 6.10747604 & 19.895 & $8.18 \mathrm{E}-06$ & 0.000766 & 91.266 & 93.433 & 77.335 & 65.557 & 45.429 & 39.4 \\
\hline Ntrk2 & -0.68419 & 6.10368989 & 19.861 & 8.33E-06 & 0.000776 & 83.106 & 77.517 & 93.106 & 48.28 & 46.554 & 63. \\
\hline 1700012P22Rik & -1.6265 & 1.67871268 & 19.713 & $9 \mathrm{E}-06$ & 0.000835 & 4.059 & 3.979 & 5.7022 & 1.7762 & 1.2114 & 1.40 \\
\hline Mcf2I & -0.72405 & 5.61800232 & 19.555 & $9.78 \mathrm{E}-06$ & 0.000903 & 74.485 & 56.296 & 51.765 & 38.753 & 34.613 & 37.4 \\
\hline Arhgef17 & -0.99669 & 4.43199471 & 19.379 & $1.07 \mathrm{E}-05$ & 0.000973 & 27.074 & 27.927 & 30.56 & 11.626 & 10.816 & 20. \\
\hline Irx3 & -0.94216 & 5.47648768 & 19.37 & $1.08 \mathrm{E}-05$ & 0.000973 & 49.964 & 51.875 & 73.326 & 27.692 & 22.585 & 40.7 \\
\hline Snord49b & -2.1244 & 1.70963344 & 19.294 & $1.12 \mathrm{E}-05$ & 0.001009 & 7.3648 & 3.758 & 3.3857 & 0.40368 & 1.6441 & 1.3 \\
\hline Pianp & -3.4256 & 3.01833087 & 19.24 & $1.15 \mathrm{E}-05$ & 0.001029 & 33.1 & 6.9264 & 1.4255 & 2.0991 & 1.0384 & 0.701 \\
\hline BC021614 & -1.8637 & 1.20652759 & 19.21 & $1.17 \mathrm{E}-05$ & 0.001036 & 3.1384 & 2.3579 & 4.5439 & 0.80735 & 0.95185 & 0.96 \\
\hline Plekha1 & -0.63298 & 7.31080803 & 19.168 & $1.2 \mathrm{E}-05$ & 0.001055 & 211.11 & 195.27 & 171.96 & 132.73 & 113.36 & 127 \\
\hline Serpina9 & -2.6392 & 0.96773105 & 19.121 & $1.23 \mathrm{E}-05$ & 0.001077 & 2.5526 & 1.4 & 5.3458 & 0.16147 & 0.51919 & 0.78 \\
\hline 9530059014Rik & -1.7855 & 1.38021318 & 19.106 & $1.24 \mathrm{E}-05$ & 0.001081 & 3.515 & 4.7896 & 2.8511 & 1.211 & 0.69226 & 1.31 \\
\hline Mpp7 & -0.69851 & 6.14415848 & 18.803 & $1.45 \mathrm{E}-05$ & 0.001251 & 90.889 & 89.823 & 81.078 & 49.975 & 44.477 & 0. \\
\hline Sepp1 & -0.84774 & 6.68329127 & 18.715 & $1.52 \mathrm{E}-05$ & 0.001305 & 158.09 & 146.63 & 90.611 & 85.256 & 71.216 & 63.3 \\
\hline Tmem159 & -0.91964 & 6.11350065 & 18.653 & 1.57E-05 & 0.001342 & 108.17 & 98.149 & 64.417 & 60.148 & 48.285 & 4. \\
\hline Mark1 & -1.2608 & 3.17139955 & 18.635 & $1.58 \mathrm{E}-05$ & 0.001349 & 13.558 & 14.148 & 9.4442 & 6.2973 & 5.8842 & 3.3 \\
\hline Iqgap2 & -1.1574 & 3.47329652 & 18.515 & $1.69 \mathrm{E}-05$ & 0.001429 & 19.96 & 11.495 & 13.454 & 7.6698 & 5.538 & 7.0 \\
\hline Rgma & -0.66672 & 5.60724969 & 18.51 & 1.69E-05 & 0.001429 & 63.48 & 63.738 & 51.408 & 37.461 & 33.055 & 42. \\
\hline Klk5 & -1.5144 & 2.85826903 & 18.464 & $1.73 \mathrm{E}-05$ & 0.001458 & 15.023 & 9.4318 & 6.4149 & 4.7634 & 3.3747 & \\
\hline Scarna13 & -0.70156 & 7.68261646 & 18.422 & $1.77 \mathrm{E}-05$ & 0.001484 & 286.89 & 259.37 & 216.15 & 152.99 & 181.37 & \\
\hline Pdzrn3 & -1.8622 & 2.77869384 & 18.396 & $1.79 \mathrm{E}-05$ & 0.001493 & 16.738 & 8.4002 & 5.524 & 4.279 & 2.596 & 1.5 \\
\hline Gpr155 & -0.84734 & 5.02061020 & 18.383 & $1.81 \mathrm{E}-05$ & 0.001498 & 49.88 & 44.948 & 29.313 & 22.687 & 21.114 & 25. \\
\hline E030018B13Rik & -5.8497 & -1.20891686 & 18.349 & $1.84 \mathrm{E}-05$ & 0.001512 & 0.71138 & 0.14737 & 0.62367 & 0 & 0 & \\
\hline Tmem229b & -1.8282 & 1.06008758 & 18.322 & $1.87 \mathrm{E}-05$ & 0.001522 & 3.2221 & 2.8737 & 2.6729 & 0.80735 & 0.43266 & 1.2 \\
\hline Atp5e & -0.7138 & 6.60848658 & 18.229 & $1.96 \mathrm{E}-05$ & 0.00158 & 132.86 & 121.07 & 108.96 & 79.766 & 85.926 & 55. \\
\hline Krtdap & -0.86041 & 8.02178830 & 18.143 & $2.05 \mathrm{E}-05$ & 0.001635 & 446.7 & 325.03 & 232.63 & 194.25 & 175.57 & 183 \\
\hline Tsix & -2.5908 & 0.60562079 & 18.141 & $2.05 \mathrm{E}-05$ & 0.001635 & 2.8037 & 0.73686 & 3.2075 & 0.48441 & 0.2596 & 0.35 \\
\hline
\end{tabular}




\begin{tabular}{|c|c|c|c|c|c|c|c|c|c|c|c|}
\hline Krt23 & -2.048 & 2.01620999 & 18.027 & $2.18 \mathrm{E}-05$ & 0.00173 & 8.0762 & 7.8844 & 2.1383 & 1.8569 & 1.298 & 1.227: \\
\hline Psph & -0.94168 & 5.88235449 & 17.996 & $2.21 \mathrm{E}-05$ & 0.001738 & 89.55 & 83.338 & 58.893 & 52.639 & 42.055 & 26.03 \\
\hline Flot2 & -0.98711 & 3.24087314 & 17.996 & $2.21 \mathrm{E}-05$ & 0.001738 & 12.512 & 12.305 & 12.117 & 6.9432 & 5.8842 & 5.785 \\
\hline Scube2 & -1.1822 & 3.50684793 & 17.826 & $2.42 \mathrm{E}-05$ & 0.001879 & 18.454 & 16.432 & 11.404 & 9.1231 & 6.5764 & 4.733 \\
\hline Ebp & -0.61016 & 5.69802244 & 17.764 & $2.5 \mathrm{E}-05$ & 0.001927 & 64.652 & 62.633 & 60.229 & 46.503 & 40.757 & 35.5 \\
\hline Nradd & -0.75548 & 4.66437731 & 17.731 & $2.54 \mathrm{E}-05$ & 0.001946 & 34.857 & 31.537 & 28.333 & 21.798 & 18.085 & 16.3 \\
\hline Hip1 & -1.2712 & 3.68781050 & 17.723 & $2.56 \mathrm{E}-05$ & 0.001948 & 17.994 & 15.621 & 20.225 & 4.6019 & 5.8842 & 11.83 \\
\hline $\mathrm{Cd} 5$ & -1.6232 & 1.55484184 & 17.527 & $2.83 \mathrm{E}-05$ & 0.002144 & 4.5193 & 4.5685 & 3.2075 & 1.0496 & 1.9037 & 1.05 \\
\hline Itgb5 & -0.69009 & 6.44611657 & 17.021 & 3.7E-05 & 0.002672 & 114.24 & 105.22 & 102.73 & 59.582 & 55.294 & 84.94 \\
\hline Sec14I2 & -0.558 & 6.53252425 & 16.761 & 4.24E-05 & 0.003002 & 116.96 & 100.58 & 112.53 & 73.63 & 69.399 & 81.26 \\
\hline Phyh & -0.58894 & 5.43693344 & 16.63 & 4.54E-05 & 0.003189 & 55.487 & 47.085 & 52.834 & 33.505 & 37.209 & 32.69 \\
\hline Rgs10 & -1.3696 & 3.53136531 & 16.161 & $5.82 \mathrm{E}-05$ & 0.003972 & 21.132 & 14.884 & 12.83 & 9.5267 & 6.4034 & 2.980 \\
\hline Cebpd & -0.71305 & 4.43859334 & 15.953 & $6.49 \mathrm{E}-05$ & 0.004389 & 23.727 & 25.348 & 31.273 & 17.197 & 15.749 & 15.86 \\
\hline Atp13a2 & -0.5578 & 6.78353742 & 15.942 & $6.53 \mathrm{E}-05$ & 0.004399 & 136.92 & 142.58 & 113.33 & 87.436 & 88.349 & 91.25 \\
\hline Pramef12 & -1.7097 & 1.02197887 & 15.917 & $6.62 \mathrm{E}-05$ & 0.004445 & 3.1803 & 3.1685 & 1.9601 & 0.72662 & 1.1249 & 0.701 \\
\hline Gns & -0.77362 & 4.76996831 & 15.847 & $6.87 \mathrm{E}-05$ & 0.004582 & 37.452 & 34.337 & 30.649 & 16.551 & 17.653 & 25.8 \\
\hline Plagl1 & -0.9014 & 3.87960651 & 15.818 & $6.98 \mathrm{E}-05$ & 0.004639 & 17.91 & 21.59 & 17.285 & 7.4276 & 11.076 & 11.9 \\
\hline $\mathrm{P} 2 \mathrm{rx} 7$ & -0.95281 & 3.29807405 & 15.766 & 7.17E-05 & 0.004738 & 13.6 & 10.905 & 13.632 & 6.3781 & 5.7976 & 7.538 \\
\hline Gm6251 & -0.70004 & 5.12081345 & 15.642 & $7.65 \mathrm{E}-05$ & 0.004979 & 51.805 & 38.611 & 37.866 & 25.512 & 30.546 & 23.14 \\
\hline Pkd113 & -2.4724 & 0.44671149 & 15.578 & $7.92 \mathrm{E}-05$ & 0.005136 & 2.971 & 0.66317 & 2.0492 & 0.32294 & 0.51919 & 0.175 \\
\hline Nupr1 & -1.2086 & 3.27435304 & 15.448 & $8.48 \mathrm{E}-05$ & 0.00545 & 18.579 & 11.2 & 9.5333 & 7.5084 & 4.8458 & 4.73 \\
\hline Fibin & -1.483 & 3.22593055 & 15.436 & 8.53E-05 & 0.005466 & 16.404 & 16.063 & 7.6623 & 6.3781 & 5.7976 & 2.15 \\
\hline Tgm1 & -1.4406 & 2.69958542 & 15.374 & $8.82 \mathrm{E}-05$ & 0.005633 & 13.516 & 8.1054 & 5.524 & 3.8753 & 2.3364 & 3.85 \\
\hline Tpm2 & -0.54133 & 6.58570172 & 15.31 & $9.12 \mathrm{E}-05$ & 0.005808 & 117.38 & 105 & 118.59 & 81.785 & 83.763 & 68.72 \\
\hline Slc39a10 & -0.51641 & 6.09948573 & 15.305 & $9.15 \mathrm{E}-05$ & 0.005808 & 80.176 & 83.486 & 77.87 & 59.825 & 56.765 & 52.2 \\
\hline Slc37a2 & -1.1035 & 3.95427137 & 15.238 & $9.48 \mathrm{E}-05$ & 0.006 & 26.907 & 24.243 & 11.137 & 9.6882 & 9.3455 & 10.08 \\
\hline Snora23 & -1.5217 & 2.66979324 & 15.189 & $9.73 \mathrm{E}-05$ & 0.006101 & 6.4442 & 14.737 & 6.3258 & 2.6643 & 2.5094 & 4.3 \\
\hline Rassf9 & -0.71439 & 5.76801814 & 15.166 & $9.85 \mathrm{E}-05$ & 0.006138 & 79.423 & 69.338 & 53.458 & 47.553 & 44.131 & 31.7 \\
\hline Ifi27|2a & -0.73358 & 4.17414565 & 15.021 & 0.000106 & 0.006539 & 24.354 & 21.295 & 21.205 & 13.483 & 15.23 & 11.5 \\
\hline Atp10b & -0.94308 & 4.50295606 & 14.994 & 0.000108 & 0.006597 & 35.945 & 31.832 & 20.67 & 12.675 & 12.72 & 20.7 \\
\hline Col8a1 & -1.2257 & 3.32628286 & 14.93 & 0.000112 & 0.006773 & 17.24 & 15.4 & 8.375 & 7.6698 & 5.9707 & 3.94 \\
\hline Apoc1 & -0.56398 & 7.16981858 & 14.925 & 0.000112 & 0.006773 & 179.94 & 167.41 & 167.32 & 135.31 & 107.47 & 105.3 \\
\hline Ltbp1 & -1.891 & 3.54811718 & 14.854 & 0.000116 & 0.007013 & 28.455 & 14.737 & 10.424 & 8.7194 & 4.4131 & 1.31 \\
\hline Gcnt2 & -0.69924 & 6.21077203 & 14.833 & 0.000117 & 0.007069 & 102.9 & 83.633 & 87.671 & 57.16 & 41.276 & 70.5 \\
\hline Dpysl5 & -1.9845 & 0.43342199 & 14.777 & 0.000121 & 0.00726 & 2.4271 & 1.7685 & 1.1583 & 0.24221 & 0.43266 & 0.701 \\
\hline Blnk & -1.0967 & 3.09494079 & 14.76 & 0.000122 & 0.007287 & 11.508 & 14.074 & 8.4641 & 4.1982 & 5.1054 & 6.662 \\
\hline E030044B06Rik & -3.8587 & -1.1113751 & 14.684 & 0.000127 & 0.007522 & 0.79507 & 0.44211 & 0.26729 & 0 & 0.086532 & \\
\hline Stbd1 & -1.9065 & 0.28683248 & 14.67 & 0.000128 & 0.00755 & 2.0923 & 1.4 & 1.2473 & 0.32294 & 0.51919 & 0.438 \\
\hline Gstm1 & -0.84973 & 3.63991943 & 14.662 & 0.000129 & 0.00755 & 14.437 & 15.253 & 17.819 & 8.1542 & 7.8744 & 10.25 \\
\hline F730043M19Rik & -1.2621 & 1.87196043 & 14.62 & 0.000132 & 0.007693 & 4.3938 & 5.379 & 4.9894 & 1.9376 & 2.0768 & 2.1 \\
\hline Gm6402 & -0.94945 & 5.77022903 & 14.601 & 0.000133 & 0.007727 & 107.04 & 54.527 & 52.923 & 36.412 & 45.602 & 29.27 \\
\hline Ift43 & -0.7129 & 4.70695289 & 14.549 & 0.000137 & 0.007899 & 38.498 & 31.169 & 26.729 & 21.314 & 20.508 & 17.1 \\
\hline Epas1 & -0.86581 & 6.05860908 & 14.539 & 0.000137 & 0.00792 & 108.51 & 89.675 & 58.982 & 59.744 & 48.198 & 33.31 \\
\hline Lmo1 & -0.71569 & 4.81583501 & 14.422 & 0.000146 & 0.008337 & 37.745 & 32.864 & 33.678 & 25.754 & 21.806 & 15.95 \\
\hline Hdac11 & -0.70242 & 4.78114503 & 14.399 & 0.000148 & 0.008408 & 36.113 & 39.127 & 26.105 & 21.233 & 22.066 & 19.1 \\
\hline
\end{tabular}




\begin{tabular}{|c|c|c|c|c|c|c|c|c|c|c|c|}
\hline Neurl1b & -0.5302 & 7.45285305 & 14.322 & 0.000154 & 0.008675 & 211.7 & 211.92 & 196.81 & 143.87 & 130.49 & 155.3 \\
\hline Ispd & -0.79917 & 3.60326008 & 14.305 & 0.000155 & 0.008727 & 15.567 & 13.927 & 16.126 & 9.1231 & 8.3071 & 8.76 \\
\hline Rgs20 & -1.239 & 2.38152658 & 14.257 & 0.000159 & 0.00893 & 7.9507 & 8.0317 & 4.9894 & 2.6643 & 3.5478 & 2.7 \\
\hline $111 \mathrm{f5}$ & -0.64306 & 5.65410543 & 14.238 & 0.000161 & 0.008997 & 70.677 & 63.517 & 49.092 & 42.224 & 42.66 & 32.69 \\
\hline BC100530 & -0.71383 & 8.48242569 & 14.183 & 0.000166 & 0.009188 & 435.61 & 350.96 & 545.98 & 237.76 & 247.74 & 326.8 \\
\hline Ctsz & -1.1593 & 3.04608953 & 14.168 & 0.000167 & 0.009205 & 12.679 & 11.937 & 8.6423 & 6.2166 & 5.7976 & 2.8 \\
\hline 2410015M20Rik & -0.70399 & 6.26614978 & 14.114 & 0.000172 & 0.009397 & 113.03 & 91.223 & 80.988 & 67.575 & 65.332 & \\
\hline Tomm7 & -0.63099 & 6.14011525 & 14.023 & 0.000181 & 0.009746 & 97.752 & 82.454 & 76.088 & 64.104 & 58.928 & 42. \\
\hline 2900060B14Rik & -0.90291 & 3.51503024 & 14.018 & 0.000181 & 0.009749 & 17.408 & 14.148 & 12.206 & 9.2845 & 6.5764 & 7.626 \\
\hline Lif & -1.4734 & 1.56537418 & 13.923 & 0.00019 & 0.010166 & 2.7618 & 4.8632 & 4.8112 & 1.6147 & 1.1249 & 1.665 \\
\hline Rgs2 & -0.78744 & 4.30794769 & 13.915 & 0.000191 & 0.010166 & 30.129 & 18.864 & 25.392 & 16.793 & 13.326 & 13.06 \\
\hline Olah & -2.6286 & -0.26282765 & 13.91 & 0.000192 & 0.010166 & 1.2972 & 1.2527 & 0.71277 & 0 & 0 & 0.525 \\
\hline Enox1 & -1.4326 & 2.77276271 & 13.865 & 0.000196 & 0.010385 & 13.265 & 10.979 & 4.3657 & 3.956 & 4.067 & 2.6 \\
\hline Ank & -0.62954 & 5.06676281 & 13.858 & 0.000197 & 0.0104 & 40.716 & 44.801 & 35.906 & 23.655 & 23.537 & 31.3 \\
\hline Cbr3 & -0.58769 & 5.42726128 & 13.77 & 0.000207 & 0.010758 & 51.847 & 53.348 & 49.181 & 36.169 & 39.112 & 27. \\
\hline Gjb4 & -1.046 & 5.23560478 & 13.727 & 0.000211 & 0.010935 & 68.334 & 53.717 & 29.045 & 31.971 & 25.267 & 16.0 \\
\hline Slc6a15 & -2.3371 & -0.31068955 & 13.726 & 0.000212 & 0.010935 & 1.3809 & 0.66317 & 0.98006 & 0.32294 & 0.086532 & 0.175 \\
\hline Kalrn & -1.2823 & 5.00138599 & 13.711 & 0.000213 & 0.010962 & 59.546 & 43.18 & 32.253 & 31.244 & 15.662 & 8.59 \\
\hline D10Bwg1379e & -1.6826 & 0.53830427 & 13.704 & 0.000214 & 0.010962 & 2.0923 & 1.5474 & 2.1383 & 0.48441 & 0.43266 & 0.876 \\
\hline Ak1 & -0.80153 & 4.96390658 & 13.698 & 0.000215 & 0.010962 & 48.081 & 31.095 & 38.935 & 30.033 & 19.729 & $18 . C$ \\
\hline Gpc4 & -0.56904 & 6.15647548 & 13.698 & 0.000215 & 0.010962 & 98.337 & 77.886 & 78.583 & 59.986 & 49.064 & \\
\hline $\mathrm{Cmbl}$ & -5.2397 & -1.64119205 & 13.648 & 0.00022 & 0.011204 & 0.16738 & 0.5158 & 0.35638 & 0 & 0 & \\
\hline Acat3 & -2.1379 & 0.91043330 & 13.638 & 0.000222 & 0.011234 & 2.72 & 1.2527 & 4.3657 & 0.64588 & 1.0384 & 0.175 \\
\hline Krt17 & -0.99119 & 9.35848871 & 13.609 & 0.000225 & 0.011357 & 1211.6 & 766.85 & 640.78 & 627.47 & 376.33 & 313 \\
\hline Lrrc8c & -1.5391 & 0.85267126 & 13.585 & 0.000228 & 0.011475 & 2.6781 & 2.579 & 1.871 & 0.80735 & 0.86532 & 0.78 \\
\hline Gulp1 & -2.2238 & -0.23491717 & 13.574 & 0.000229 & 0.011506 & 1.4228 & 1.0316 & 0.71277 & 0.24221 & 0.34613 & 0.087 \\
\hline Heph & -0.51765 & 6.77146766 & 13.553 & 0.000232 & 0.011587 & 137.97 & 127.92 & 119.3 & 97.528 & 79.177 & 92.4 \\
\hline Ceacam2 & -3.0632 & -1.10046814 & 13.546 & 0.000233 & 0.0116 & 0.50215 & 0.5158 & 0.62367 & 0 & 0 & 0.175 \\
\hline Skint4 & -1.0912 & 5.80688397 & 13.475 & 0.000242 & 0.011963 & 102.77 & 78.107 & 46.508 & 55.142 & 25.7 & 25.9 \\
\hline Mllt4 & -0.57992 & 8.83851318 & 13.385 & 0.000254 & 0.012513 & 612.87 & 548.29 & 483.7 & 362.42 & 334.19 & 404 \\
\hline Fabp5 & -0.61816 & 6.96801049 & 13.354 & 0.000258 & 0.012643 & 143.95 & 168.15 & 142.11 & 89.858 & 124.35 & 81. \\
\hline Sn $\times 22$ & -1.5807 & 3.34433017 & 13.341 & 0.00026 & 0.012697 & 25.819 & 7.1475 & 11.137 & 5.3285 & 6.836 & 2.6 \\
\hline Hes1 & -0.51136 & 6.38776433 & 13.283 & 0.000268 & 0.01298 & 109.51 & 90.265 & 94.709 & 70.885 & 64.207 & 1. \\
\hline Mir703 & -0.9481 & 3.79551687 & 13.275 & 0.000269 & 0.013001 & 21.843 & 14.737 & 17.374 & 9.6882 & 12.201 & 6.1 \\
\hline Bglap3 & -2.5934 & 0.07071951 & 13.263 & 0.000271 & 0.013054 & 2.0923 & 1.7685 & 0.26729 & 0.16147 & 0.34613 & 0.17 \\
\hline Rnf122 & -0.88426 & 3.13133422 & 13.193 & 0.000281 & 0.01349 & 11.842 & 10.095 & 11.404 & 6.1359 & 5.2784 & 6.66 \\
\hline Lama4 & -1.1526 & 3.90662293 & 13.177 & 0.000283 & 0.013575 & 20.128 & 12.748 & 28.511 & 12.433 & 5.4515 & \\
\hline Adamts5 & -2.3604 & 0.81208501 & 13.167 & 0.000285 & 0.013589 & 4.059 & 2.6527 & 0.62367 & 0.72662 & 0.17306 & 0.52 \\
\hline Skint2 & -1.7678 & 1.48785762 & 13.098 & 0.000296 & 0.013967 & 4.5193 & 5.8212 & 1.5146 & 0.96882 & 1.1249 & \\
\hline Fkbp1a & -0.68563 & 4.51574887 & 13.092 & 0.000297 & 0.013979 & 30.547 & 31.758 & 21.472 & 16.631 & 17.826 & \\
\hline Nrep & -1.1318 & 3.42287541 & 13.083 & 0.000298 & 0.014013 & 15.943 & 16.506 & 10.781 & 9.1231 & 7.0956 & 3.5 \\
\hline Slc9a7 & -1.6979 & 0.70149916 & 13.069 & 0.0003 & 0.01409 & 1.7994 & 1.7685 & 3.2075 & 0.72662 & 0.43266 & \\
\hline Cachd1 & -0.50654 & 7.45579661 & 13.065 & 0.000301 & 0.01409 & 218.85 & 211.04 & 187.55 & 138.7 & 142.35 & 153 \\
\hline Ptn & -0.4758 & 5.91347411 & 12.986 & 0.000314 & 0.014628 & 69.38 & 67.054 & 73.415 & 53.285 & 51.313 & 46. \\
\hline Lrrfip1 & -0.49813 & 5.76473053 & 12.974 & 0.000316 & 0.014689 & 69.548 & 58.506 & 62.189 & 44.243 & 47.766 & 42. \\
\hline
\end{tabular}




\begin{tabular}{|c|c|c|c|c|c|c|c|c|c|c|c|}
\hline Pcsk5 & -1.7706 & 2.10205773 & 12.953 & 0.000319 & 0.014817 & 9.6664 & 5.6738 & 3.1184 & 3.0679 & 1.471 & 0.876 \\
\hline Fn1 & -1.1276 & 4.86398028 & 12.946 & 0.000321 & 0.014817 & 61.639 & 37.138 & 19.69 & 21.233 & 13.672 & \\
\hline Cox8a & -0.61612 & 6.83394104 & 12.916 & 0.000326 & 0.015018 & 150.64 & 135.07 & 127.76 & 94.622 & 107.73 & 67.49 \\
\hline Cygb & -1.3458 & 1.35236437 & 12.884 & 0.000331 & 0.015245 & 3.4732 & 3.979 & 2.6729 & 1.3725 & 1.471 & 1.1 \\
\hline Apex1 & -0.62045 & 6.30415627 & 12.836 & 0.00034 & 0.015576 & 104.78 & 92.991 & 88.74 & 74.034 & 66.543 & 45.8 \\
\hline Slc41a2 & -2.1621 & 1.17089406 & 12.805 & 0.000346 & 0.015766 & 2.3852 & 1.6948 & 6.3258 & 1.0496 & 0.17306 & 1.0 \\
\hline Atxn7|1 & -0.76806 & 4.05081290 & 12.785 & 0.000349 & 0.015901 & 20.546 & 19.527 & 21.918 & 11.868 & 9.0859 & 15.42 \\
\hline Ndufa4 & -0.79918 & 6.48742389 & 12.776 & 0.000351 & 0.015924 & 144.33 & 111.27 & 85.265 & 78.313 & 74.677 & 43.04 \\
\hline Foxg1 & -2.6698 & 3.47871460 & 12.771 & 0.000352 & 0.015924 & 45.528 & 7.5159 & 1.871 & 2.9872 & 3.9805 & 1.665 \\
\hline Myh2 & -2.3722 & -0.32582749 & 12.739 & 0.000358 & 0.016122 & 0.83691 & 0.73686 & 1.6928 & 0.24221 & 0 & 0.3506 \\
\hline Hadh & -0.53094 & 7.40980989 & 12.716 & 0.000363 & 0.016285 & 207.76 & 212.66 & 181.85 & 136.28 & 157.4 & 123 \\
\hline Cyp17a1 & -1.2833 & 2.67018060 & 12.669 & 0.000372 & 0.016594 & 11.508 & 9.137 & 5.2567 & 3.5523 & 2.3364 & 4.82 \\
\hline Rps15a-ps6 & -0.7092 & 5.37251385 & 12.622 & 0.000381 & 0.016977 & 58.417 & 51.138 & 43.835 & 40.045 & 31.671 & 22. \\
\hline Hdac7 & -0.76103 & 5.29287899 & 12.596 & 0.000387 & 0.017178 & 49.587 & 46.053 & 51.587 & 19.942 & 26.133 & 40.8 \\
\hline Crot & -0.49185 & 6.53235971 & 12.515 & 0.000404 & 0.017782 & 111.69 & 116.57 & 95.6 & 77.829 & 82.032 & \\
\hline Ttc22 & -0.62276 & 4.31461191 & 12.512 & 0.000404 & 0.017782 & 25.944 & 24.537 & 21.116 & 14.936 & 15.662 & $16 . C$ \\
\hline 1134 & -0.55219 & 5.31466380 & 12.438 & 0.000421 & 0.018301 & 46.7 & 51.211 & 43.39 & 27.45 & 32.969 & 36. \\
\hline Snord33 & -3.4833 & -1.3827301 & 12.337 & 0.000444 & 0.019244 & 0.41846 & 0.5158 & 0.26729 & 0 & 0.086532 & \\
\hline Gm5148 & -1.8476 & 2.19913873 & 12.313 & 0.00045 & 0.019405 & 9.8337 & 3.3159 & 7.1277 & 2.5028 & 2.6825 & 0.438 \\
\hline Slc39a8 & -0.51771 & 6.35227208 & 12.301 & 0.000453 & 0.019491 & 94.488 & 110.68 & 82.77 & 62.57 & 70.437 & \\
\hline Qrich2 & -1.5416 & 1.10804288 & 12.287 & 0.000456 & 0.019606 & 3.2221 & 3.3895 & 2.0492 & 0.64588 & 1.5576 & 0.788 \\
\hline Lphn2 & -0.57966 & 6.546666003 & 12.275 & 0.000459 & 0.019648 & 132.69 & 108.1 & 94.353 & 81.462 & 61.351 & \\
\hline Ccl27a & -0.82556 & 6.255598837 & 12.24 & 0.000468 & 0.019936 & 109.13 & 115.83 & 67.179 & 70.643 & 58.149 & 36. \\
\hline Gas7 & -0.83922 & 5.40896471 & 12.187 & 0.000481 & 0.020427 & 69.338 & 47.232 & 45.974 & 44.001 & 24.662 & \\
\hline Ifitm3 & -0.76376 & 6.30752092 & 12.17 & 0.000486 & 0.020532 & 132.48 & 93.36 & 72.168 & 72.096 & 60.053 & 43. \\
\hline C1qtnf7 & -1.5427 & 0.75936631 & 12.143 & 0.000493 & 0.020793 & 2.4271 & 1.5474 & 2.762 & 0.72662 & 0.60572 & 0.96 \\
\hline Pglyrp4 & -1.6229 & 3.11726169 & 12.13 & 0.000496 & 0.020851 & 18.119 & 15.695 & 4.0093 & 4.9248 & 5.2784 & 2 \\
\hline Bnc2 & -0.73385 & 3.62958131 & 12.051 & 0.000518 & 0.021453 & 14.646 & 14.811 & 16.305 & 8.3964 & 9.0859 & .95 \\
\hline Gm10677 & -1.5564 & 0.71703630 & 11.897 & 0.000562 & 0.023115 & 2.4689 & 1.6948 & 2.3165 & 0.96882 & 0.86532 & 0.35 \\
\hline Map2 & -0.69767 & 5.17099462 & 11.876 & 0.000569 & 0.023252 & 47.704 & 42.222 & 43.033 & 25.593 & 19.383 & \\
\hline $\mathrm{Ccl} 8$ & -1.2225 & 2.16058599 & 11.875 & 0.000569 & 0.023252 & 5.7747 & 5.8212 & 6.4149 & 1.6954 & 2.1633 & \\
\hline Arl15 & -0.5192 & 5.34874020 & 11.862 & 0.000573 & 0.023371 & 51.428 & 50.548 & 41.252 & 32.375 & 32.969 & 34.8 \\
\hline Tbx18 & -3.6675 & -0.55746389 & 11.845 & 0.000578 & 0.023534 & 1.7994 & 0.44211 & 0.089096 & 0.16147 & 0 & \\
\hline Snord12 & -1.9012 & -0.22204903 & 11.779 & 0.000599 & 0.02406 & 0.96245 & 1.4 & 0.89096 & 0.32294 & 0.2596 & 0.262 \\
\hline Fxyd5 & -1.6935 & 0.21354576 & 11.779 & 0.000599 & 0.02406 & 1.9249 & 1.2527 & 1.1583 & 0.48441 & 0.43266 & 0.4 \\
\hline 4930473A02Rik & -2.2085 & 0.14319899 & 11.701 & 0.000625 & 0.024993 & 2.0923 & 0.5158 & 1.7819 & 0.080735 & 0.51919 & 0.35 \\
\hline Igsf10 & -1.5103 & 1.23744893 & 11.675 & 0.000633 & 0.025251 & 4.352 & 2.9474 & 2.0492 & 0.96882 & 0.77879 & 1.57 \\
\hline Tec & -0.71423 & 4.70349269 & 11.671 & 0.000635 & 0.025251 & 39.753 & 30.506 & 25.927 & 24.463 & 16.614 & 7. \\
\hline Prss12 & -1.154 & 1.89329918 & 11.671 & 0.000635 & 0.025251 & 4.4775 & 4.9369 & 5.2567 & 1.8569 & 1.9037 & 2.80 \\
\hline Snora7a & -1.0089 & 2.42871678 & 11.612 & 0.000655 & 0.02592 & 7.1556 & 7.737 & 5.8803 & 3.7138 & 3.5478 & .0 \\
\hline Lipg & -0.80558 & 3.72833069 & 11.594 & 0.000662 & 0.026054 & 19.249 & 18.127 & 12.295 & 10.576 & 7.9609 & 9.95 \\
\hline Dctpp1 & -0.71797 & 4.60612106 & 11.592 & 0.000662 & 0.026054 & 36.782 & 28.664 & 24.501 & 20.83 & 20.335 & 13.6 \\
\hline Srgn & -2.9934 & -1.12410776 & 11.588 & 0.000664 & 0.026058 & 0.62769 & 0.58948 & 0.26729 & 0 & 0 & 0.17 \\
\hline Dgat2 & -0.6858 & 4.723399970 & 11.57 & 0.00067 & 0.026256 & 35.82 & 36.548 & 24.501 & 17.6 & 18.518 & 24.2 \\
\hline Adamts18 & -2.0382 & 0.49139956 & 11.563 & 0.000673 & 0.026268 & 1.5064 & 0.88423 & 3.742 & 0.40368 & 0.34613 & 0.70 \\
\hline
\end{tabular}




\begin{tabular}{|c|c|c|c|c|c|c|c|c|c|c|c|}
\hline Сур7b1 & -2.309 & 0.96900575 & 11.556 & 0.000675 & 0.026309 & 1.8412 & 1.5474 & 5.7913 & 0.080735 & 0.51919 & 1.227 \\
\hline Fzd3 & -1.1205 & 4.05796208 & 11.527 & 0.000686 & 0.026621 & 29.418 & 23.285 & 14.612 & 15.097 & 10.73 & 5.171 \\
\hline Tmem180 & -1.5876 & 0.27348107 & 11.49 & 0.0007 & 0.027013 & 1.632 & 1.5474 & 1.4255 & 0.64588 & 0.60572 & 0.26 \\
\hline Ado & -0.48291 & 6.38232597 & 11.46 & 0.000711 & 0.0274 & 97.375 & 103.16 & 90.611 & 78.071 & 68.62 & 61.6 \\
\hline Syt12 & -0.86989 & 3.61983182 & 11.446 & 0.000717 & 0.027538 & 18.287 & 13.484 & 15.057 & 11.707 & 7.0956 & 6.837 \\
\hline Spata13 & -1.4502 & 1.33714667 & 11.439 & 0.000719 & 0.027538 & 4.3101 & 3.1685 & 2.5838 & 1.7762 & 1.298 & 0.61 \\
\hline Pcbd1 & -1.7438 & 0.41128330 & 11.437 & 0.00072 & 0.027538 & 2.5526 & 1.3263 & 1.1583 & 0.40368 & 0.69226 & 0.438 \\
\hline Ggh & -0.63714 & 6.64138130 & 11.393 & 0.000737 & 0.028049 & 141.52 & 133.74 & 88.383 & 89.051 & 82.984 & 61. \\
\hline Pnpla1 & -0.79539 & 5.00792241 & 11.293 & 0.000778 & 0.02927 & 53.521 & 43.622 & 24.234 & 23.978 & 20.941 & 25.2 \\
\hline Clic3 & -0.85768 & 3.88998822 & 11.122 & 0.000853 & 0.031595 & 23.434 & 20.927 & 11.939 & 11.707 & 10.73 & \\
\hline Ung & -0.61727 & 4.38069970 & 11.071 & 0.000877 & 0.032408 & 25.693 & 23.579 & 25.749 & 16.712 & 19.21 & 12.5 \\
\hline Mrpl41 & -0.56081 & 4.70278650 & 11.057 & 0.000883 & 0.032504 & 32.723 & 29.253 & 30.471 & 22.929 & 21.72 & 18.05 \\
\hline Terc & -0.97164 & 3.89336108 & 11.056 & 0.000884 & 0.032504 & 13.516 & 22.769 & 22.274 & 11.303 & 12.72 & 5.6 \\
\hline Angpt1 & -2.0069 & 0.34033586 & 11.033 & 0.000895 & 0.032735 & 1.3809 & 3.0948 & 0.71277 & 0.40368 & 0.60572 & 0.2629 \\
\hline Eif4ebp1 & -0.60814 & 4.67662825 & 11.004 & 0.000909 & 0.033201 & 34.439 & 31.39 & 26.016 & 22.444 & 21.027 & 16.5 \\
\hline Insig2 & -0.53484 & 5.94510951 & 10.967 & 0.000928 & 0.033645 & 81.474 & 73.98 & 62.456 & 61.116 & 45.083 & 44.3 \\
\hline Mir3074-1 & -2.9183 & -1.1684016 & 10.963 & 0.00093 & 0.033645 & 0.66953 & 0.44211 & 0.26729 & 0.080735 & 0 & 0.087 \\
\hline Atp6v1b2 & -0.49805 & 5.78974370 & 10.94 & 0.000941 & 0.033999 & 69.757 & 69.264 & 54.527 & 49.491 & 40.93 & 46 \\
\hline Pdzd4 & -0.4875 & 5.15195146 & 10.93 & 0.000946 & 0.034138 & 43.31 & 42.296 & 38.222 & 31.81 & 29.075 & 27.52 \\
\hline Ninj1 & -0.68759 & 4.03057187 & 10.888 & 0.000968 & 0.034785 & 23.015 & 18.053 & 18.621 & 15.017 & 11.163 & 10.5 \\
\hline Nop10 & -0.57906 & 5.25099439 & 10.865 & 0.00098 & 0.035035 & 49.755 & 49.075 & 37.242 & 34.393 & 32.19 & 24.6 \\
\hline Adamts9 & -1.1135 & 2.99068066 & 10.847 & 0.00099 & 0.03521 & 12.261 & 8.2528 & 11.226 & 7.5084 & 4.4131 & 2.71 \\
\hline Hebp2 & -0.53405 & 5.97075729 & 10.808 & 0.00101 & 0.035764 & 80.72 & 80.465 & 60.585 & 50.863 & 59.274 & 43.2 \\
\hline Fscn1 & -0.48345 & 5.45610128 & 10.808 & 0.001011 & 0.035764 & 51.679 & 44.58 & 56.754 & 35.523 & 35.911 & 37.9 \\
\hline Astn2 & -3.6179 & -0.5780098 & 10.805 & 0.001012 & 0.035764 & 1.7575 & 0.5158 & 0 & 0.080735 & 0 & 0.087 \\
\hline Pkib & -0.68854 & 4.55810429 & 10.786 & 0.001023 & 0.036019 & 33.435 & 31.685 & 21.205 & 20.507 & 14.537 & 18.67 \\
\hline Nme1 & -0.61495 & 6.14660382 & 10.768 & 0.001033 & 0.036277 & 99.76 & 82.159 & 74.395 & 62.812 & 65.245 & 39.4 \\
\hline Gstp1 & -0.5974 & 4.67760664 & 10.767 & 0.001034 & 0.036277 & 34.272 & 28.811 & 28.6 & 23.009 & 21.2 & 16 \\
\hline Rbms3 & -1.2407 & 3.93399139 & 10.761 & 0.001037 & 0.036327 & 27.702 & 23.064 & 12.473 & 14.694 & 8.0475 & 4.03 \\
\hline Bzw2 & -0.51892 & 6.79530244 & 10.756 & 0.001039 & 0.036357 & 150.35 & 130.72 & 110.57 & 99.062 & 96.656 & 77.8 \\
\hline Fdft1 & -0.55001 & 5.85459737 & 10.752 & 0.001042 & 0.036386 & 83.357 & 70.37 & 51.587 & 49.571 & 46.035 & 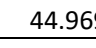 \\
\hline Atp5o & -0.56768 & 7.06823611 & 10.73 & 0.001054 & 0.036752 & 185.29 & 155.33 & 139.35 & 125.54 & 115.17 & 83.2 \\
\hline Icam2 & -1.9391 & -0.44874724 & 10.711 & 0.001065 & 0.036853 & 0.83691 & 0.81054 & 1.0692 & 0.16147 & 0.17306 & $0.35 c$ \\
\hline Fhdc1 & -0.44462 & 6.24538890 & 10.711 & 0.001065 & 0.036853 & 93.483 & 88.865 & 79.296 & 62.085 & 64.812 & \\
\hline Aldh3b2 & -0.5985 & 6.31588324 & 10.706 & 0.001068 & 0.036853 & 117.63 & 99.77 & 69.495 & 70.401 & 63.428 & 55.9 \\
\hline Rarres1 & -0.92021 & 4.66483306 & 10.703 & 0.00107 & 0.036853 & 26.112 & 31.169 & 41.786 & 11.949 & 13.153 & 27.1 \\
\hline Tcf4 & -0.43772 & 6.07507490 & 10.703 & 0.00107 & 0.036853 & 76.494 & 78.475 & 77.157 & 52.559 & 55.986 & 62 \\
\hline Dnm3 & -1.1564 & 2.69344153 & 10.7 & 0.001071 & 0.036853 & 9.7501 & 8.0317 & 8.1078 & 2.745 & 2.5094 & 3 \\
\hline Txnrd3 & -0.46276 & 5.97912817 & 10.613 & 0.001123 & 0.038448 & 77.959 & 73.317 & 67.357 & 59.502 & 52.611 & 46.6 \\
\hline Galt & -0.52552 & 4.95287714 & 10.585 & 0.00114 & 0.038846 & 38.665 & 38.095 & 32.164 & 27.208 & 26.219 & 22. \\
\hline Sardh & -1.4242 & 1.18571662 & 10.563 & 0.001154 & 0.039122 & 3.3477 & 2.3579 & 3.3857 & 1.4532 & 1.471 & 0.43 \\
\hline Capn2 & -0.78262 & 6.57262303 & 10.557 & 0.001157 & 0.039176 & 117.42 & 95.497 & 147.63 & 58.614 & 47.852 & 103 \\
\hline Nme4 & -1.0425 & 1.93500979 & 10.518 & 0.001182 & 0.039637 & 5.3563 & 4.6422 & 4.633 & 2.5835 & 2.1633 & 2.3 \\
\hline Fbln2 & -1.6683 & 0.81720876 & 10.492 & 0.001199 & 0.040044 & 3.5987 & 1.2527 & 2.0492 & 1.0496 & 0.51919 & 0.6136 \\
\hline Stxbp6 & -1.3718 & 1.12709477 & 10.49 & 0.0012 & 0.040044 & 3.7243 & 2.579 & 2.1383 & 1.3725 & 1.2114 & 0.70 \\
\hline
\end{tabular}




\begin{tabular}{|c|c|c|c|c|c|c|c|c|c|c|c|}
\hline Ccr2 & -3.6462 & -1.19945372 & 10.49 & 0.0012 & 0.040044 & 0.87876 & 0.22106 & 0.17819 & 0 & 0 & 0.0876 \\
\hline Tomm5 & -0.52017 & 5.13687748 & 10.482 & 0.001206 & 0.040131 & 44.482 & 38.759 & 40.45 & 31.406 & 31.152 & 23.75 \\
\hline Сур27a1 & -0.84982 & 3.82810998 & 10.48 & 0.001207 & 0.040131 & 18.538 & 16.063 & 19.512 & 14.209 & 9.3455 & 6.399 \\
\hline Pycard & -0.54687 & 7.60325655 & 10.468 & 0.001215 & 0.040234 & 245.8 & 238.67 & 207.42 & 172.45 & 179.73 & 121. \\
\hline Angptl1 & -4.5753 & -1.95962314 & 10.467 & 0.001216 & 0.040234 & 0.25107 & 0.14737 & 0.17819 & 0 & 0 & \\
\hline Bola2 & -0.63301 & 4.66815278 & 10.416 & 0.001249 & 0.041022 & 33.477 & 30.801 & 27.709 & 21.556 & 23.277 & 14.55 \\
\hline Rnu11 & -0.69128 & 3.94289578 & 10.382 & 0.001272 & 0.041646 & 20.672 & 19.821 & 15.681 & 11.061 & 14.018 & 9.817 \\
\hline Nipal1 & -0.97702 & 2.83657539 & 10.372 & 0.00128 & 0.041825 & 11.926 & 8.6212 & 6.8604 & 5.8129 & 3.8074 & 4.38 \\
\hline Lypd5 & -1.9532 & -0.16417976 & 10.368 & 0.001282 & 0.041835 & 1.2972 & 1.5474 & 0.44548 & 0.24221 & 0.2596 & 0.3506 \\
\hline Uqcr10 & -0.66929 & 5.99082141 & 10.356 & 0.001291 & 0.041922 & 93.4 & 76.265 & 63.704 & 60.955 & 53.304 & 32.60 \\
\hline Cacnb4 & -2.1016 & -0.45951801 & 10.34 & 0.001302 & 0.042227 & 1.2554 & 0.73686 & 0.53458 & 0.24221 & 0.086532 & 0.2629 \\
\hline Slc39a2 & -1.3581 & 2.71703180 & 10.333 & 0.001307 & 0.042317 & 11.508 & 11.495 & 4.1875 & 5.5707 & 2.8556 & 2.191 \\
\hline Bmp7 & -0.43559 & 5.89394169 & 10.325 & 0.001313 & 0.042424 & 76.243 & 65.875 & 62.189 & 49.733 & 51.66 & 49.96 \\
\hline Paqr8 & -1.1773 & 1.29679112 & 10.259 & 0.00136 & 0.043767 & 3.6406 & 2.9474 & 2.762 & 1.3725 & 1.298 & 1.490 \\
\hline Cox6b2 & -1.8455 & -0.37652433 & 10.233 & 0.001379 & 0.044279 & 1.0043 & 0.81054 & 0.98006 & 0.32294 & 0.34613 & 0.0876 \\
\hline Isyna1 & -0.60988 & 4.69712523 & 10.232 & 0.00138 & 0.044279 & 34.062 & 34.485 & 24.68 & 23.252 & 20.595 & 17.35 \\
\hline Sptb & -1.5607 & 2.08370151 & 10.203 & 0.001402 & 0.044918 & 4.1009 & 3.0948 & 11.315 & 2.0184 & 1.3845 & 2.805 \\
\hline Mrpl55 & -0.72111 & 3.94594043 & 10.191 & 0.001411 & 0.045065 & 23.517 & 16.211 & 16.928 & 12.837 & 12.461 & 9.204 \\
\hline Itgb8 & -1.455 & 3.79424449 & 10.132 & 0.001457 & 0.046243 & 27.409 & 19.969 & 12.295 & 13.079 & 6.663 & 2.016 \\
\hline Ostc & -0.55365 & 5.81071730 & 10.13 & 0.001459 & 0.046243 & 72.686 & 66.833 & 60.051 & 51.428 & 51.4 & 33.22 \\
\hline Gm6548 & -0.74895 & 6.91707501 & 10.124 & 0.001464 & 0.046283 & 227.72 & 114.58 & 111.1 & 99.466 & 92.676 & 77.9 \\
\hline Cox6b1 & -0.6047 & 6.82034343 & 10.079 & 0.001499 & 0.047229 & 163.74 & 132.27 & 112.17 & 105.76 & 97.175 & 65.65 \\
\hline Trpm4 & -0.45699 & 5.61803849 & 10.072 & 0.001505 & 0.047342 & 53.855 & 52.833 & 63.347 & 39.641 & 39.805 & 44.26 \\
\hline Rpl39 & -0.58443 & 9.27183977 & 10.049 & 0.001525 & 0.047669 & 835.66 & 759.48 & 629.11 & 571.77 & 519.28 & 392.4 \\
\hline Cat & -0.64997 & 6.36640274 & 10.049 & 0.001525 & 0.047669 & 132.23 & 98.149 & 70.921 & 76.941 & 55.38 & 59.95 \\
\hline Pyy & -1.5281 & 0.94721100 & 10.04 & 0.001532 & 0.04783 & 3.5569 & 2.579 & 1.3364 & 1.0496 & 0.51919 & 1.051 \\
\hline Snrpd3 & -0.69662 & 4.06415058 & 10.026 & 0.001543 & 0.048111 & 24.061 & 22.474 & 14.612 & 11.707 & 13.326 & 12.88 \\
\hline Scel & -0.53809 & 8.80933311 & 10.013 & 0.001555 & 0.048313 & 626.26 & 545.72 & 421.07 & 365.57 & 346.73 & 385.0 \\
\hline Hoxc8 & -0.62127 & 3.87345495 & 10.007 & 0.001559 & 0.048345 & 18.998 & 16.211 & 17.374 & 12.11 & 10.816 & 11.30 \\
\hline Pcsk6 & -0.65327 & 5.69329852 & 10.006 & 0.00156 & 0.048345 & 83.399 & 63.001 & 42.321 & 45.212 & 37.468 & 37.60 \\
\hline Fgfbp1 & -0.50292 & 7.82660279 & 9.9896 & 0.001574 & 0.04871 & 306.69 & 256.79 & 234.23 & 211.69 & 186.74 & 164.7 \\
\hline Cers2 & -0.60243 & 4.04633395 & 9.9659 & 0.001595 & 0.049123 & 22.095 & 19.674 & 17.196 & 12.433 & 13.412 & 13.14 \\
\hline Ndufa12 & -0.61666 & 5.13679580 & 9.9616 & 0.001598 & 0.049163 & 50.173 & 42.59 & 34.124 & 31.002 & 31.325 & 20. \\
\hline
\end{tabular}

LogFC $=$ Log Fold change, $\log C P M=$ Log count per million bases, LR $=$ Log Ratio, FDR $=$

BH corrected P-value, values in columns S7309-S7314 depict TMM normalized relative expression. 
Supplementary Table S2 Pathway analysis of upregulated (A) and downregulated (B) genes in Lgr6 $^{+}$basal epidermal keratinocytes.

A.

\begin{tabular}{|c|c|c|c|c|c|}
\hline Term & Genes & Count & $\%$ & P-Value & $\begin{array}{l}\text { Benjamini } \\
\text { Hochberg } \\
\text { corrected }\end{array}$ \\
\hline Pathways in cancer & $\begin{array}{l}\text { FGFR2, WNT16, FGFR3, PDGFB, WNT3A, } \\
\text { ADCY6, FGF13, LAMB3, WNT4, PLCB4, } \\
\text { AXIN2, MMP1B, BMP4, COL4A4, COL4A3, } \\
\text { BMP2, WNT10B, COL4A2, TGFBR1, ITGA2, } \\
\text { KITL, COL4A6, WNT7B, LAMA3, LAMC2, } \\
\text { LAMC1, PTCH2 }\end{array}$ & 27 & 9,4 & $1,70 \mathrm{E}-10$ & $2,90 \mathrm{E}-08$ \\
\hline Amoebiasis & $\begin{array}{l}\text { COL4A4, GNA14, COL4A3, IL1R1, COL4A2, } \\
\text { COL4A6, LAMB3, PLCB4, LAMA3, } \\
\text { SERPINB2, HSPB1, LAMC2, LAMC1 }\end{array}$ & 13 & 4,5 & $1,90 \mathrm{E}-07$ & $1,60 \mathrm{E}-05$ \\
\hline $\begin{array}{l}\text { Hedgehog signaling } \\
\text { pathway }\end{array}$ & $\begin{array}{l}\text { BMP4, WNT16, WNT10B, BMP2, WNT7B, } \\
\text { WNT4, WNT3A, GAS1, PTCH2 }\end{array}$ & 9 & 3,1 & $5,40 \mathrm{E}-07$ & 3,10E-05 \\
\hline Basal cell carcinoma & $\begin{array}{l}\text { BMP4, WNT16, WNT10B, BMP2, WNT7B, } \\
\text { WNT4, WNT3A, PTCH2, AXIN2 }\end{array}$ & 9 & 3,1 & 1,60E-06 & $6,80 \mathrm{E}-05$ \\
\hline Small cell lung cancer & $\begin{array}{l}\text { COL4A4, COL4A3, COL4A2, LAMB3, LAMA3, } \\
\text { ITGA2, LAMC2, LAMC1, COL4A6 }\end{array}$ & 9 & 3,1 & $3,90 \mathrm{E}-05$ & $1,30 \mathrm{E}-03$ \\
\hline $\begin{array}{l}\text { Signaling pathways } \\
\text { regulating pluripotency } \\
\text { of stem cells }\end{array}$ & $\begin{array}{l}\text { FGFR2, BMP4, WNT16, WNT10B, BMP2, } \\
\text { WNT7B, WNT4, FGFR3, WNT3A, LIFR, } \\
\text { AXIN2 }\end{array}$ & 11 & 3,8 & 4,60E-05 & $1,30 \mathrm{E}-03$ \\
\hline $\begin{array}{l}\text { ECM-receptor } \\
\text { interaction }\end{array}$ & $\begin{array}{l}\text { COL4A4, COL4A3, COL4A2, LAMB3, LAMA3, } \\
\text { ITGA2, LAMC2, LAMC1, COL4A6 }\end{array}$ & 9 & 3,1 & 5,50E-05 & $1,30 \mathrm{E}-03$ \\
\hline $\begin{array}{l}\text { PI3K-Akt signaling } \\
\text { pathway }\end{array}$ & $\begin{array}{l}\text { FGFR2, COL4A4, COL4A3, COL4A2, FGFR3, } \\
\text { PDGFB, OSMR, ITGA2, FGF13, KITL, IRS1, } \\
\text { COL4A6, LAMB3, LAMA3, LAMC2, NGFR, } \\
\text { LAMC1 }\end{array}$ & 17 & 5,9 & $8,10 \mathrm{E}-05$ & $1,70 \mathrm{E}-03$ \\
\hline $\begin{array}{l}\text { Cytokine-cytokine } \\
\text { receptor interaction }\end{array}$ & $\begin{array}{l}\text { IL12RB2, IL1R1, CXCL14, PDGFB, OSMR, } \\
\text { CCR4, IL20RA, TGFBR1, LIFR, EDAR, NGFR, } \\
\text { KITL, IL22RA2 }\end{array}$ & 13 & 4,5 & $3,40 \mathrm{E}-04$ & $6,40 \mathrm{E}-03$ \\
\hline $\begin{array}{l}\text { Hippo signaling } \\
\text { pathway }\end{array}$ & $\begin{array}{l}\text { BMP4, WNT16, WNT10B, BMP2, WNT7B, } \\
\text { WNT4, CTGF, TGFBR1, WNT3A, AXIN2 }\end{array}$ & 10 & 3,5 & 4,60E-04 & 7,90E-03 \\
\hline
\end{tabular}


B.

\begin{tabular}{|c|c|c|c|c|c|}
\hline Term & Genes & Count & $\%$ & P-Value & $\begin{array}{l}\text { Benjamini } \\
\text { Hochberg } \\
\text { corrected }\end{array}$ \\
\hline Metabolic pathways & $\begin{array}{l}\text { LDHB, ATP5E, GCNT2, OLAH, ACOT1, } \\
\text { ITPKB, ADH7, ATP6V1B2, PSPH, CMBL, } \\
\text { FDFT1, ALDH3A1, ISYNA1, UQCR10, } \\
\text { CERS2, COX6B1, ATP5O, COX6B2, HADH, } \\
\text { SARDH, BDH1, IMPDH1, DCTPP1, NDUFA4, } \\
\text { EBP, AK1, COX8A, GALT, EPHX2, ALDH3B2, } \\
\text { CBR3, NDUFA12, GNS, NME4, CYP17A1, } \\
\text { ADO, CYP27A1, DGAT2, NME1, LIPG, AOX4 }\end{array}$ & 41 & 10,6 & $3,30 \mathrm{E}-05$ & $6,00 \mathrm{E}-03$ \\
\hline $\begin{array}{l}\text { Metabolism of } \\
\text { xenobiotics by } \\
\text { cytochrome P450 }\end{array}$ & $\begin{array}{l}\text { GSTM1, ALDH3B2, ADH7, CBR3, GSTM5, } \\
\text { GSTP1, ALDH3A1 }\end{array}$ & 7 & 1,8 & 9,70E-05 & 8,90E-03 \\
\hline $\begin{array}{l}\text { Drug metabolism - } \\
\text { cytochrome P450 }\end{array}$ & $\begin{array}{l}\text { GSTM1, ALDH3B2, AOX4, ADH7, GSTM5, } \\
\text { GSTP1, ALDH3A1 }\end{array}$ & 7 & 1,8 & $1,40 \mathrm{E}-04$ & 8,60E-03 \\
\hline $\begin{array}{l}\text { Oxidative } \\
\text { phosphorylation }\end{array}$ & $\begin{array}{l}\text { NDUFA4, ATP5E, UQCR10, COX8A, } \\
\text { COX6B1, ATP5O, ATP6V1B2, COX6B2, } \\
\text { NDUFA12 }\end{array}$ & 9 & 2,3 & $2,30 \mathrm{E}-03$ & $1,00 \mathrm{E}-01$ \\
\hline Alzheimer's disease & $\begin{array}{l}\text { NDUFA4, ATP5E, UQCR10, COX8A, GRIN1, } \\
\text { COX6B1, ATP5O, COX6B2, CAPN2, } \\
\text { NDUFA12 }\end{array}$ & 10 & 2,6 & 2,90E-03 & $1,00 \mathrm{E}-01$ \\
\hline
\end{tabular}


Supplementary Table S3 Primers used for qPCR.

\begin{tabular}{|c|c|c|c|c|c|c|}
\hline Gene & Primer sequence (5'-> 3') & $\begin{array}{l}\text { Amplicon } \\
\text { size }\end{array}$ & $\begin{array}{l}\text { Exonic } \\
\text { Location }\end{array}$ & Accession No. & $\begin{array}{l}\text { Amplification } \\
\text { efficiency }\end{array}$ & $\begin{array}{c}\text { Correlation } \\
\left(\mathbf{R}^{2}\right)\end{array}$ \\
\hline \multirow[t]{2}{*}{ Cyc1 } & GATGTCGCCACCTTCCTTCGC & \multirow[t]{2}{*}{136} & \multirow[t]{2}{*}{6,7} & \multirow[t]{2}{*}{ NM_025567.2 } & \multirow[t]{2}{*}{$98.6 \%$} & \multirow[t]{2}{*}{0.99} \\
\hline & GGACTGACCACTTATGCCGCTTC & & & & & \\
\hline \multirow[t]{2}{*}{ Ddx52 } & CACAGCAGCAGCGAGATAACAC & \multirow[t]{2}{*}{177} & \multirow{2}{*}{$\begin{array}{l}\text { 10-11 } \\
11-12\end{array}$} & \multirow[t]{2}{*}{ NM_030096 } & \multirow[t]{2}{*}{$107.0 \%$} & \multirow[t]{2}{*}{0.99} \\
\hline & CTCTTCCAGTCCGACCTATCCTATG & & & & & \\
\hline \multirow{2}{*}{$\begin{array}{l}\text { Lgr6 } \\
\text { ex1-2 }\end{array}$} & GCTGTCCGCTGACTGCTC & \multirow[t]{2}{*}{102} & \multirow[t]{2}{*}{1,2} & \multirow[t]{2}{*}{ NM_001033409 } & \multirow[t]{2}{*}{$95.0 \%$} & \multirow[t]{2}{*}{0.97} \\
\hline & AGCTCCGTGAGGTTGTTCAT & & & & & \\
\hline \multirow{2}{*}{$\begin{array}{l}\text { Lgr6 } \\
\text { ex1-3 }\end{array}$} & GGACCCCCTGACGGCTTA & \multirow[t]{2}{*}{120} & \multirow[t]{2}{*}{1,3} & \multirow[t]{2}{*}{ NM_001033409 } & \multirow[t]{2}{*}{$95.0 \%$} & \multirow[t]{2}{*}{0.97} \\
\hline & TGTGAGAGGTGGTTCCCTGA & & & & & \\
\hline \multirow{2}{*}{$\begin{array}{l}\text { Lgr6 } \\
\text { ex3-4 }\end{array}$} & TCAGGGAACCACCTCTCACA & \multirow[t]{2}{*}{113} & \multirow[t]{2}{*}{3,4} & \multirow[t]{2}{*}{ NM_001033409 } & \multirow[t]{2}{*}{$92.0 \%$} & \multirow[t]{2}{*}{0.95} \\
\hline & CATAGTGCCTCTGCTGGGAT & & & & & \\
\hline \multirow[t]{2}{*}{ Mki67 } & CAGAAGAAATGGAAGCCAAAA & \multirow[t]{2}{*}{110} & \multirow[t]{2}{*}{12,13} & \multirow[t]{2}{*}{ NM_001081117.2 } & \multirow[t]{2}{*}{$96.5 \%$} & \multirow[t]{2}{*}{0.99} \\
\hline & TGCAGATGCATCAAACTTGG & & & & & \\
\hline
\end{tabular}

Supplementary Table S4. Sample information RNA-seq

\begin{tabular}{|c|c|c|c|c|}
\hline $\begin{array}{l}\text { Mouse } \\
\text { code }\end{array}$ & Gender & Genotype & $\begin{array}{c}\text { Age } \\
\text { (days) }\end{array}$ & sample IDs \\
\hline 701 & M & Lgr6-EGFP-IRES-CreERT2 heterozygous & 28 & \multirow{2}{*}{$7309 / 7310$} \\
\hline 721 & $\mathrm{~F}$ & Lgr6-EGFP-IRES-CreERT2 heterozygous & 31 & \\
\hline 720 & M & Lgr6-EGFP-IRES-CreERT2 heterozygous & 31 & \multirow{2}{*}{$7311 / 7312$} \\
\hline 722 & $\mathrm{~F}$ & Lgr6-EGFP-IRES-CreERT2 heterozygous & 31 & \\
\hline 565 & M & Lgr6-EGFP-IRES-CreERT2 heterozygous & 95 & \multirow{2}{*}{$7313 / 7314$} \\
\hline 774 & $\mathrm{~F}$ & Lgr6-EGFP-IRES-CreERT2 heterozygous & 90 & \\
\hline 508 & $\mathrm{M}$ & Lgr6-EGFP-IRES-CreERT2 homozygous & 147 & \multirow{2}{*}{$7315 / 7316$} \\
\hline 550 & $\mathrm{~F}$ & Lgr6-EGFP-IRES-CreERT2 homozygous & 176 & \\
\hline
\end{tabular}

